\title{
Abstracts of the papers presented in the XXVI conference of Indian virological society, "Viruses to Viromes in Health and Disease", during 07-09 December, 2017, at Nitte University, Deralakatte, Mangalore-575018, India
}

(C) Indian Virological Society 2018

\section{Shyama Prasad Raychaudhuri Lifetime Achievement Award}

Challenges in prevention and management of dengue virus infection

\section{A. C. Mishra}

Email ID for Correspondence: acm1750@gmail.com Interactive Research School of Health Affairs, Bharati Vidyapeeth University, Katraj, Pune 411043, India

Dengue is an important emerging public health problem in countries of Tropical and Subtropical regions. Estimated burden of disease is about 390 million infections, 96 million clinical cases, and 20,000 deaths. However, country wise specific disease burden data is lacking. The virus belongs to the genus Flavivirus, family Flaviviridae. It has 4 antigenically distinct serotypes (DENV1, DENV-2, DENV-2, DENV4). The virus is transmitted through mosquito bite. Aedes aegypti is the principal vector but A. albopictus has also been found to play role in some areas. The disease spectrum includes subclinical infection, mild cases and severe cases. Severe cases may include Shock, haemorrhage, severe organ involvements resulting into death. Pathology of the disease is poorly understood. Treatment for dengue is supportive only. No antiviral agents have been discovered. Currently there is no licensed vaccine. Vaccines are in different stages of development. Prevention of dengue is primarily vector control centered around outbreaks with little impact in real term.

\section{Kameshwar Sahai Bhargava Oration Award}

Viral disease diagnosis: from symptomatology to next generation sequencing

\section{R. K. Jain}

Email ID for Correspondence: rakeshjain56@yahoo.co.in Indian Agricultural Research Institute, New Delhi-12, India

Plant viruses are the major limiting factors affecting the cultivation of field as well as horticultural crops. Rapid and reliable diagnosis is essential to minimise the losses caused by viruses. Diagnostic virology has received considerable attention during the last 2-3 decades and an array of diagnostic methods ranging from symptomatology to next generation sequencing (NGS) with varying detection limit (100-100 ng) are available. While the large-scale adoption of immuno- and nucleobased diagnostic methods such as enzyme-linked immunosorbent assay (ELISA) and polymerase chain reaction (PCR) has resulted in early and accurate virus diagnosis, these techniques fail to detect unknown viruses for which prior information is not available. Although bioassay using indicator plants and electronmicroscopy are available to detect the unknown viruses, these are slow and not amenable to large-scale sampling. More recently, multiplexed methods for simultaneous detection of viruses such as multiplex ELISA/PCR, macro- and micro-array and NGS have been developed. Of these, NGS is generic (non-targeted) and facilitates virome analysis of both plants as well as vectors.

\section{Medical Virology}

\section{Oral Presentation}

Herpes virus genomics

\section{Andrew J. Davison}

Email ID for Correspondence: Andrew.davidson@glasgow.ac.uk MRC-University of Glasgow Centre for Virus Research, Glasgow, United Kingdom

Herpesviruses are classified into three families in the order Herpesvirales. Members of the Herpesviridae infect mammals, birds or reptiles, members of the Alloherpesviridae infect amphibians or fish, and members of the Malacoherpesviridae infect bivalves or gastropods. Our understanding of the genomics of these viruses has been invigorated in recent years by high-throughput DNA sequencing. As a result, the field has moved from a position of being dominated by a few laboratory strains, some of which are not typical of the clinical agent, to one in which extensive data are available on viral strains as they exist in nature. Nowhere are these advances more apparent than with human cytomegalovirus (HCMV), which can be a problem in congenital or transplant-acquired infections and also in people infected with HIV. The viral genome $(236,000 \mathrm{bp})$ has several features of interest in genomic studies. These include the existence of hyper variable genes, the presence of natural gene-disrupting 
mutations, the prevalence of infections involving multiple strains, an evolutionary history characterised by extensive recombination, and a propensity to mutate when isolated in cell culture. As a result, meaningful studies necessarily include analysing strains directly from clinical material. By using modified Illumina sequencing library preparation and target enrichment techniques coupled to a bioinformatics pipeline for sequence assembly, we have now analysed the HCMV genomes present in a large number of clinical samples from various sources. We have incorporated into this pipeline an assessment of whether the sequencing libraries represent the sample adequately, as well as the genotyping of hyper variable genes aimed at identifying the number of strains present. This presentation will overview the state of herpes virus genomics and illustrate how findings in the HCMV field are leading to a deeper understanding of the scope and role of sequence diversity in the evolution, expression and pathogenesis of this herpes virus.

\section{Exploiting APOBEC3 proteins to elucidate HIV-1 nuclear import} and develop gene therapy treatment

\section{Vinay K. Pathak}

Email ID for Correspondence: pathakv@mail.nih.gov Viral Mutation Section, HIV Dynamics and Replication ProgramNCI, Frederick, Maryland 02

Human genomes encode seven APOBEC3 restriction proteins as a defense against HIV-1 and other pathogens. In the absence of viral Vif protein, APOBEC $3 \mathrm{G}(\mathrm{A} 3 \mathrm{G})$ and $\mathrm{APOBEC} 3 \mathrm{~F}(\mathrm{~A} 3 \mathrm{~F})$ are packaged into virions in the virus producing cells and inhibit viral replication in the infected target cells by inducing lethal G-to-A hypermutation. Vif binds to the A3 proteins and targets them for proteasomal degradation, allowing viral replication to proceed. We have exploited the fact that $\mathrm{A} 3 \mathrm{G} / \mathrm{A} 3 \mathrm{~F}$ proteins are packaged into virions to visualize viral complexes in infected cells and gained novel insights into the dynamics and regulation of HIV-1 nuclear import and its intranuclear movements after import. Fluorescently-tagged virion-incorporated A3F or HIV-1 integrase were used to visualize viral complexes in infected cells with high-resolution fluorescence microscopy. We determined the dynamics of HIV-1 docking with the nuclear envelope, visualized nuclear import, and analyzed intranuclear movements of viral complexes. We observed that unlike cellular nuclear proteins, viral complexes exhibited unusually long residence times at the nuclear envelope $(1.5 \pm 1.6 \mathrm{~h})$, and that the nuclear docking and import were dependent on HIV-1 capsid (CA) and nuclear pore protein Nup358. Furthermore, translocation of viral complexes into the nucleus was associated with substantial loss of CA, indicating that one of the viral core uncoating steps occurs during nuclear import. We also found that interaction of CA with host protein cyclophilin A delays nuclear import by slowing down nuclear envelope docking and transport through the nuclear pore. We also found that after nuclear import viral complexes exhibited a brief fast phase of nuclear movement $(<9 \mathrm{~min})$, followed by a long slow phase lasting several hours; a comparison of the movements of nuclear viral complexes and integrated HIV-1 DNA suggests that the viral complexes become tethered to chromatin soon after nuclear import. Using lentiviral vectors to deliver and express Vif-resistant mutant of A3G (A3GD128K) in HIV-1 target cells could potently inhibit replication and spread of HIV-1 and facilitate development of a functional cure for HIV-1 infection. We have constructed and analyzed HIV-1 vectors that can deliver and express $\mathrm{A} 3 \mathrm{G}-\mathrm{D} 128 \mathrm{~K}$ to $\mathrm{T}$ cell lines, primary $\mathrm{CD}^{+}{ }^{+} \mathrm{T}$ cells and $\mathrm{CD} 34^{+}$human stem cells. We found that expression of A3G-D128K in $\mathrm{T}$ cell lines potently inhibited replication of HIV-1 subtypes B, C, and AE. Proviral DNA in the infected cells was extensively hypermutated, indicating inhibition by A3G-128K. No
A3G-D128K resistant HIV-1 was selected after several months in cell culture, indicating that evolving resistance to A3G-D128K represents a high genetic barrier. We hypothesize thatevolving resistance may be difficult because Vif must retain the ability to induce degradation of wild-type A3G, A3F, A3D, and perhaps other A3 proteins. These studies establish the feasibility of expressing Vif-resistant A3GD128K in HIV-1 target cells using gene therapy to achieve a functional cure for HIV-1 infection.

Molecular multiplex testing for respiratory viruses and its impact on patient care and outcomes

\section{Rangaraj Selvarangan}

Email ID for Correspondence: rselvarangan@cmh.edu Director, Clinical Microbiology, Virology and Molecular Infectious Diseases Laboratory, Department of Pathology and Laboratory Medicine, Children's Mercy Kansas City, UMKC School of Medicine

Acute respiratory infections are one of the most common respiratory illnesses in children and adults worldwide. Majority of these infections are caused by viruses with increasing burden and severity of illness most commonly occurring in infants, elderly and the immunocompromised patients. Rapid and accurate detection of the etiological agent helps in patient treatment and management decisions. This lecture will describe laboratory tests currently available for respiratory virus detection and discuss the strengths and weaknesses of traditional and molecular laboratory tests for respiratory pathogens. The advent of multiplex molecular assays has enabled the laboratory to test for multiple pathogens from a single respiratory specimen. This in turn has increased the diagnostic yield of the respiratory specimen testing and provides opportunity for efficient antibiotic and antiviral treatment decisions, increased patient satisfaction, improved infection control practices, reduced patient stay and overall health care savings. Experience with outbreak detection and management of respiratory viral infection will be shared. Clinical studies and local data measuring the impact of these respiratory pathogen molecular detection assays in patient outcomes will be discussed with review of the strengths and limitation of the findings. The need for these clinical impact studies will be emphasized and study designs for future investigations will be presented.

\section{Hepatitis $\mathrm{C}$ virus persistence, liver disease progression and prevention strategies}

\section{Ranjit Ray}

Email ID for Correspondence: ranjit.ray@ health.slu.edu Division of Infectious Diseases, Allergy and Immunology, Departments of Internal Medicine, Molecular Microbiology and Immunology, Saint Louis University, Missouri, USA

Chronic hepatitis $\mathrm{C}$ virus (HCV) infection often causes end stage liver disease. Although current anti-HCV drugs are promising, they do not prevent reinfection or end stage liver disease progression. Understanding the mechanisms of liver disease progression is necessary for novel treatment strategies. There is no vaccine available against HCV. Therefore, acomprehensive strategy for effective vaccine development is needed to control HCV infection. HCV induces CCL5 secretion from macrophages/Kupffer cells, activates hepatic stellate cells, generates epithelial mesenchymal transition state/tumor initiating cancer stem-like cells, and induces local fibroblast activation by secretion of TGF- $\beta$. Molecular aspects of these findings will be discussed in context to liver pathogenesis. In our vaccine development 
effort against HCV infection, recent studies suggested that HCV E2 envelope glycoprotein establishes bias for macrophage activation toward the $\mathrm{M} 2$ phenotype, impairs $\mathrm{DC} / \mathrm{CD} 4^{+} \mathrm{T}$ cell development and functions, and modulates $\mathrm{C} 3$ complement component with the potential for impairment of T-helper cell function. We are working for evaluation of selected HCV E2 epitopes together with E1 and NS regions for immunogenicity and cross-protective immuneresponses to HCV genotypes in preclinical animal models. Taken together, these results will help us in rational design of therapeutic modalities and $\mathrm{HCV}$ vaccine trial in humans.

New insights into arbovirus disease mechanisms: a focus on Zika virus and alphaviruses

\section{Suresh Mahalingam}

Email ID for Correspondence: s.mahalingam@griffith.edu.au Principal Research Leader and Head, Emerging Viruses and Inflammation Research Group, Institute for Glycomics, Griffith University, Queensland, Australia

Virus-induced inflammatory diseases, including arthritis, pneumonia and encephalitis, are a significant cause of human morbidity and mortality. There is a clear need to improve our understanding of the pathogenesis of this diverse array of conditions. In recent years my laboratory has unravelled the mechanisms of how arboviruses such as chikungunya and Zika viruses cause disease. Arboviruses have caused explosive epidemics involving millions of patients, with several recently emerging in new geographic locations. My laboratory has carried out extensive work in understanding the pathogenesis of arbovirus disease and identifying new mechanisms, which has led to the identification of new candidate therapies for viral infections.

\section{Gastroenteric pathogens and blood-type connection}

B. V. Venkataram Prasad ${ }^{1,2}$, L. Hu1, S. Shanker ${ }^{1}$, S. Ramani' ${ }^{2}$, S. E. Crawford ${ }^{2}$, J-M. Choi ${ }^{1}$, R. L. Atmar ${ }^{2}$, and M. K. Estes ${ }^{2}$

Email ID for Correspondence: vprasad@bcm.edu

${ }^{1}$ Verna and Marrs McLean Department of Biochemistry and Molecular Biology; ${ }^{2}$ Department of Molecular Virology and Microbiology, Baylor College of Medicine, Houston, TX 77030

Initial attachment to host cells is a critical event in the life cycle of a virus and involves recognition by the viruses of specific receptors on the cell surface, including glycans. Viruses that utilize glycans as receptors typically exhibit strain-dependent variations in glycan specificity, a feature that contributes significantly to cell tropism, host specificity, host adaptation and interspecies transmission. Both rotaviruses and noroviruses are well known gastroenteric pathogens that are of significant global health concern. Rotaviruses, in the family Reoviridae, are the major causative agents of life-threatening diarrhea in children, whereas noroviruses, which belong to Caliciviridae family, cause epidemic and sporadic cases of acute gastroenteritis across all age groups. Both groups of viruses exhibit enormous genotypic and serotypic diversity. Consistent with this diversity, each exhibits strain-dependent variations in the types of glycans they recognize for cell attachment. In recent years, using a combination of electron cryomicroscopy, X-ray crystallography, glycan microarray screening, and cell-based functional assays, we have made paradigmshifting discoveries that have led to a better understanding of how these human pathogens exploit polymorphic histo-blood group antigens, which are the determinants of blood type, for the initial cell attachment. This talk will focus on how such strain-dependent variations in glycan recognition by these human pathogens impact host tropism, susceptibility to infection, zoonosis and vaccine development. This work is supported by grants from NIH (R37 AI36040, R01 AI080656, R01 AI105101, PO1 AI057788), and Robert Welch foundation (Q1279).

\section{Measles Rubella surveillance in India}

\section{Lucky Sangal}

Email ID for Correspondence: sangallu@who.int Laboratory Focal Point WHO Country Office for India

India has set the goal for measles elimination and rubella/CRS control by 2020 . As a part of this endeavour, India is implementing strategies to strengthen routine immunization coverage with measles vaccine and laboratory based measles and rubella surveillance system across the country. Laboratory supported measles and rubella surveillance system has been initiated and expanded in phase manner. Since 2015, the MR surveillance system is established across all states and union territories covering the entire population in India. The measles and rubella surveillance is outbreak based where the flagged outbreaks are investigated and line list is generated for all those suspected measles cases that are falling under the outbreak. The robust platform of AFP surveillance system was used to strengthen the measles and rubella surveillance. Modified case cased MR surveillance first becomes functional in Karnataka in 2016. Rapid expansion of modified case based surveillance to other states of India is happening. The MR surveillance data generated from outbreak investigations and laboratory confirmation provides key epidemiological evidence on measles and rubella transmission across different states in the country. The time, place, person epidemiology including vaccination status of confirmed measles and rubella cases could be done for strategic actions. The information has been very useful in driving programmatic policies and decision making regarding measles elimination and rubella control in the country.

\section{Rotavirus vaccine development: the India story}

\section{Sudhanshu Vrati}

Email ID for Correspondence: vrati@nii.res.in Regional Centre for Biotechnology, Faridabad 121001

Rotavirus is the leading cause of diarrhoea-associated hospitalisations and deaths in developing countries, estimated to account for approximately 610,000 deaths annually. It is a particularly important problem in India where nearly one-fourth of these deaths occur and it is estimated that about 1 child in 250 in India will die of this disease. Accordingly, development of vaccines for rotavirus infections has been accorded high priority. As part of the Indo-US Vaccine Action Program, a Department of Biotechnology (DBT), Government of India sponsored activity to promote new vaccine development, two rotavirus strains were identified that demonstrated interesting properties as potential candidate vaccines. These strains were obtained from outbreaks of asymptomatically infected newborns in Delhi (116E) and Bangalore (I321). 116E is a human strain with a single gene segment encoding VP4 derived from a bovine rotavirus. By contrast, strain I321 was a bovine strain with two non-structural gene segments derived from a human strain. Safety and immunogenicity studies of two orally administered human rotavirus AGMK celladapted vaccine candidates $116 \mathrm{E}$ and $\mathrm{I} 321$ were carried out in ninety healthy infants aged 8 weeks who received a single dose of $116 \mathrm{E}$ $\left(10^{5} \mathrm{ffu}\right)$, I321 $\left(10^{5} \mathrm{ffu}\right)$ or placebo. There were no significant 
differences in the number of adverse events. These studies showed that $116 \mathrm{E}$ strain was attenuated, clinically safe and highly immunogenic. The neonatal rotavirus candidate vaccine $116 \mathrm{E}$ was subsequently tested in a double-blind, placebo-controlled dose escalation trial in India. Two doses of the Vero cell-adapted vaccine were evaluated. There were no vaccine-related serious adverse events. A fourfold increase in rotavirus IgA titer was observed in 66.7 and $64.5 \%$ of infants after the first administration and in 62.1 and $89.7 \%$ of infants after 3 administrations of doses of $10^{4} \mathrm{ffu}$ and $10^{5} \mathrm{ffu}$, respectively. Subsequently, a randomised double-blind, placebocontrolled, multicentre trial of $10^{5} \mathrm{ffu} 116 \mathrm{E}$ vaccine was conducted at three sites in Delhi (urban), Pune (rural), and Vellore (urban and rural) that enrolled 6799 infants aged 6-7 weeks. Of these, 4532 infants were assigned to receive the $116 \mathrm{E}$ vaccine and 2267 to receive placebo. The incidence of severe rotavirus gastroenteritis per 100 person-years was 1.5 in the vaccine group and 3.2 in the placebo group. Prevalence of immediate, solicited, and serious adverse events was similar in both groups. These studies demonstrated that the monovalent human-bovine (116E) rotavirus vaccine was effective and well tolerated in Indian infants. Having established the safety and efficacy, the $116 \mathrm{E}$ rotavirus vaccine 'Rotavac' produced by BBIL, Hyderabad was launched by the Prime Minister of India for use in India.

\section{Advancements in the immunopathogenesis of Japanese encephalitis: more to explore}

\section{Ratho R.K.}

Email ID for Correspondence: rathopgi@yahoo.com

Department of Virology, Postgraduate Institute of Medical Education and Research, PGIMER, Chandigarh, India

Japanese encephalitis remained to be an important arboviral disease in India and South East Asian countries being transmitted by Culex group of mosquitoes. Because of neurotropic in nature it is one of the core members within the umbrella of Acute Encephalitis Syndrome (AES). Recent years, JE has posed a public health threat in many states of Indian sub-continent including Haryana, Gorakhpur in Eastern UP etc. JE is an enveloped virus with SS, +sense, RNA of $\sim 11 \mathrm{~kb}$ length. Single serotype circulates in nature and of the 4 genotypes; genotype 3 is the circulating strain in Indian subcontinent. Normally the virus gets transmitted between the Culex mosquitoes and the birds like ponds herons and cattle egrets where pigs serve as the amplifying host and human beings are the accidental host. JE presents with sub-clinical to the life threatening acute meninoencephalitis with cortical damage and cord lesions resulting 10-45\% mortality. Following an outbreak of Japenese encephalitis one-third of patients end with fatal outcome, complete recovery in one-third whereas the other third experience with neurological sequelae like speech impairment, learning, behavioral changes and motor paresis. The pathogenesis in flavivirus encephalitis might be a combination of tissue damage, host antibody response against E, NS1 and NS3 proteins and the cellular response through cytotoxic T-lymphocytes. The differential expression of pro and anti-inflammatory cytokines in blood and CSF has been correlated with disease severity. High levels of interferon- $\gamma$ (IFN- $\gamma$ ), interleukin-6 (IL-6), tumor necrosis factoralpha (TNF- $\alpha$ ) and interleukin-2 (IL-2) have been reported in JE cases. IFN- $\gamma$ mediated antigen presentation through up-regulation of MHC class-I and II and co-stimulatory molecules further leading to the activation and differentiation of Tc cells as well as helper lymphocytes with Th1 cytokine profile. Increased production of TNF- $\alpha$ was associated with neurological complications in JE. TNF- $\alpha$ levels significantly elevated in encephalitis patients as compared to fever group and healthy controls. Among the eight known SNPs known within the TNF promoter region, $-308 \mathrm{~A}$ and $-863 \mathrm{C}$ alleles are more vulnerable to the severe form of JE.E protein often referred to be a genetic determinant of virulence in animal models of flavivirus encephalitis. The region is under constant immune selective pressure and provides sufficient evolutionary and epidemiological relationships. A novel mutation-S227T corresponding the loop region of domain 2 of $\mathrm{E}$ gene of JEV in comparison to Indian and other isolates has been observed. In an independent study, S123R mutation in E protein exhibited a significant increase in virulence in a mice model. Thus, it is very pertinent to explore the interplay between human host, vector mosquitoes, environment and the genetic changes in the virus itself for better understanding the disease pathogenesis of JE.

\section{Following the tracks of kyasanur forest disease (KFD) in India}

\section{Arunkumar G}

Email ID for Correspondence: arun.kumar@manipal.edu Manipal Centre for Virus Research, Manipal University, India

Kyasanur forest diseases (KFD) is a tick-borne flavivirus disease first described in 1957 from Shimoga district of Karnataka, India. KFD detection remained restricted to persons living in forest regions of Shimoga and adjoining districts, until 2012 when it was identified in Chamarajanagar, $400 \mathrm{~km}$ away from its first detection. Characteristically, this disease is described as an acute haemorrhagic febrile illness. After an intense phase of exhaustive studies after the discovery, the research on KFD was abruptly concluded in 1960's due to various reasons. In 2014 we initiated the hospital-based laboratorysupported acute febrile illness (AFI) surveillance at sub-district level sentinel hospitals across several states to map the aetiology of AFI in general and KFD in particular. All admitted AFI patients from June 2014 to July 2017 with fever $<15$ days were enrolled. We recorded the demographic and clinical parameters of all cases, and tested for bacterial, viral and parasitic diseases, including leptospirosis, dengue, influenza, scrub typhus, chikungunya, typhoid, brucellosis, and KFD. Serological and molecular diagnostic assays were performed, including real-time PCR to detect viral RNA in serum for KFD confirmation. We enrolled 20,104 AFI patients from five contiguous Indian states along Western Ghats. Of these, 606 (3\%) were KFDpositive: Karnataka (151/8109), Kerala (46/3689), Tamil Nadu (16/ 4953), Goa (313/2229), and Maharashtra (80/1124). KFD-positive patients ranged from 2 to 65 years (median age 40 years); $56 \%$ were female. Their clinical spectrum included myalgia $(88 \%)$, generalized weakness (84\%), prostration (20\%), nausea/vomiting (50\%), abdominal pain $(30 \%)$, diarrhea $(24 \%)$, hemorrhagic fever $(1 \%)$, and altered sensorium and or seizures (1\%). Of 606 cases, $82 \%$ were living near the forest edge and $81 \%$ reported visiting the forest in the last 2 weeks. This study documents that KFD is not restricted to the Shimoga forest region but is prevalent in other regions along the Western Ghats. The clinical presentation of the disease is more diverse than previously observed. Further, in our surveillance, wehave also recorded cases without confirmed forest incursion. In-depth ecological studies are needed to understand the distribution and maintenance of KFD in nature to develop evidence based cost effective public health measures for control of the disease.

Rotavirus viroplasm structures: the first insights into the organizational assembly of viral and host factors

\section{Durga Rao C}

Email ID for Correspondence: cdrmcb@gmail.com 
Department of Microbiology and Cell Biology, Indian Institute of Science, Bangalore 560012

Rotavirus genome replication and assembly of immature doublelayered particles (DLPs) occur in specialized cytoplasmic inclusions called viroplasms (VMs), the precise composition and structural organization of which is not yet understood. Further, studies on the association of cellular proteins with VM and their role in rotavirus biology remained largely unexplored. Here, employing various methods, we demonstrate rotavirus-induced cytoplasmic relocalization of a large number of nuclear heterogeneous nuclear ribonucleoproteins (hnRNPs), AU-rich element-binding proteins (ARE-BPs), and transport factors. Significantly, while the levels of some cellular proteins are severely reduced, that of some are enhanced in rotavirus infected cells, suggesting that the virus modulates the expression and stability of specific cellular proteins. Majority of the relocalized proteins as well as some cytoplasmic proteins are sequestered by the viroplasmic proteins NSP2 and NSP5 in the VM through direct or indirect interactions, forming inclusions referred here to as viroplasm structures (VSs). High-resolution confocal microscopy revealed that many small unit VMs (u-VMs) consisting of NSP5 and NSP2, formed during 4-5 h post infection, associated with other viral and host proteins to become unit VSs (u-VSs), several of which associate into barrel-like large VSs (1-VSs) in which the viral and several cellular proteins assume ring-like structures that are organized proximal to distal in the order NSP5-NSP2-VP1-VP6, forming a central channel with broader proximal NSP5 end and tapering distal VP6 end. The cellular proteins are organised between NSP5 and NSP2 or NSP2 and VP6. The major findings involving cytoplasmic relocalization of a large number of hnRNPs and AREBPs in infected cells, the positive or negative influence of cellularproteins on virus growth, and the complex composition and spatial organization of viral and host factors in the VS, which remained ununderstood so far, will be discussed.

\section{Molecular mechanism of differential neuroAIDS by HIV-1 B and $\mathbf{C}$ clades}

\section{Shailendra K Saxena ${ }^{1,2}$}

Email ID for Correspondence: shailen@kgmcindia.edu

${ }^{1}$ Center for Advanced Research (CFAR)-Stem Cell/Cell Culture Unit, King George's Medical University (KGMU), Lucknow 226003,

India; ${ }^{2}$ CSIR-Centre for Cellular and Molecular Biology, Uppal Road, Hyderabad 500007, India

Human immunodeficiency virus type 1 (HIV-1) is commonly associated with immune dysfunctions and the suppression of antigenpresenting cells. This results in immune alterations, which could lead to impaired neuronal functions, such as neuroAIDS. The neurotoxic factor kynurenine (KYN), the rate limiting enzyme indoleamine 2,3dioxygenase (IDO), serotonin (5-HT), and serotonin transporter (5HTT) may play a role in tryptophan deficiency and serotogenic dysfunction in neuroAIDS. HIV-1 transactivator regulatory protein (Tat) is known to play a major role in immune dysfunction. Previous studies suggest that HIV-1 B and C clades differentially manifest neuronal dysfunctions in the infected host. In this study we examined the effect of HIV-1 B and C clade-derived Tat on IDO and 5-HTT, to understand molecular mechanism of differential neuroAIDS by HIV$1 \mathrm{~B}$ and $\mathrm{C}$ clades. In the present study, we examined the effect of HIV$1 \mathrm{~B}$ and $\mathrm{C}$ clade-derived Tat on IDO and 5-HTT gene and protein expressions by dendritic cells as studied by quantitative polymerase chain reaction (qPCR) and Western blot. In addition, the intracellular IDO expression, IDO enzyme activity, and the levels of 5-HT and KYN were also measured. Our results indicate that HIV-1 clade B Tat up-regulates IDO and down-regulates 5-HTT gene and protein expressions. Further, HIV-1 clade B Tat caused a reduction of 5-HT with simultaneous increase in KYN levels as compared to HIV-1 clade C Tat. These studies suggest that HIV-1 clade B and C Tat proteins may play a differential role in the neuropathogenesis of HIVassociated dementia (HAD) or HIV-associated neurocognitive disorder (HAND).

\section{Zika infection: public health threat to India}

\section{Gajanan N Sapkal}

Email ID for Correspondence: gajanansapkalniv@gmail.com Diagnostic Virology Group National Institute of Virology, MCC, 130/1, Pashan, Sus Road, Pune - 411021, Maharashtra

Zika virus (ZIKV) is an emerging mosquito-borne pathogen that has been designated a global health threat by the WHO due to its gestational impact on the developing central nervous system. In 1954 National Institute of Virology, Pune (NIV) (formerly known as Virus Research Center) had tested samples from Bharuch district, which showed ZIKV antibody detection in $16.8 \%$ of the samples. However, due to high cross-reactivity of ZIKV with Dengue and other flaviviruses, it was difficult to confirm Zika infection in India based on serology. Laboratory based surveillance for Zika virus was launched in India in February 2016, following World Health Organization (WHO) declaration of Public Health Emergency of International Concern (PHEIC). More than 35,000 serum samples of febrile illness have been tested which yielded three cases identified in Gujarat and one in Chennai did not reveal any travel history to ZIKV endemic region, suggesting that the ZIKV is not a recent introduction into the country and it may have been present as a vector-borne entity albeit in a silent, low key ecological niche. In addition, about 18,000 mosquitoes have been tested, this includes about 500 mosquitoes from the Bapunagar area in Ahmadabad, where two of the Zika cases were reported; but the virus could not be detected. In the context of ZIKV epidemiology, there are still some major concerns in the affected countries viz. travel related ZIKV introductions, the specific symptoms, risks, prevention and clinical management of the individuals, clinical aspects of the affected pregnant women and associated microcephaly.

\section{Enhancing surveillance for acute encephalitis syndrome in India}

V. Ravi ${ }^{1}$, Anita Desai ${ }^{1}$, Reeta Mani', Ravi Yadav', Amita Jain ${ }^{2}$, Lahari Saikia $^{3}$, Borthakur $\mathrm{AK}^{3}$, Daiji Gogoi ${ }^{4}$, Ajanta Sharma ${ }^{4}$, Nemai Bahttacharya ${ }^{5}$, Bhaswati Bandophadhyay, Shafeeque S Hameed ${ }^{1}$, Vijayalakshmi Reddy ${ }^{1}$, Anoop Velayudhan 6 , Sen $\mathbf{P K}^{7}$, Khasnobis $\mathbf{P}^{8}$, Dhariwal $\mathbf{A C}^{8}$, Jagadish Prasad ${ }^{9}$, Kayla Laserson ${ }^{6}$ and Padmini Srikantaiah ${ }^{6}$

Email ID for Correspondence: virusravi@gmail.com ${ }^{1}$ Departments of Neurovirology and Neurology, NIMHANS, Bangalore-560029; ${ }^{2}$ Department of Microbiology King George Medical University, Lucknow; ${ }^{3}$ Department of Microbiology, Assam Medical College, Dibrugarh, Assam ${ }^{4}$ Department of Microbiology, Guwahati Medical College, Assam; ${ }^{5}$ Department of Virology, School of Tropical Medicine, Kolkota; ${ }^{7}$ National Vector Borne Disease Control Program, New Delhi; ${ }^{8}$ National Centre for Disease Control, New Delhi; ${ }^{9}$ Director General of Health Services, Nirman Bhavan, New Delhi; ${ }^{6} \mathrm{CDC}$, India Office, New Delhi

Acute Encephalitis Syndrome (AES) is a major public health problem in India. Although Japanese encephalitis virus (JEV) is the major 
cause of AES identified in India (ranging from 5 to 35\%), no systematic efforts have been made to determine the precise etiology of all AES cases. During the past 3 years, we established a tiered network of quality assured laboratories for JE testing in 20 district hospitals of four states which have been linked to six Apex laboratories in (1-2 per state). These apex labs have the capability for detecting eight Non-JE pathogens (4-bacterial, 4-viral) which are known to be causative agents for AES in India. In addition, an effective sample referral mechanism with defined turnaround time for testing and reporting has been achieved within the network. This has resulted in the etiological investigation of over 10,000 cases of AES. $\mathrm{JE}$ is the commonest cause of AES detected amongst these cases $(16 \%)$, investigated in the network. The major value addition provided by the network established in this project has been the detection of other bacterial/viral etiological agents contributing to AES (24\%). Generation of this vital information would not have been possible in the NVBDCP surveillance system existent in the country prior to the initiation of this project. The detection of treatable bacterial causes of AES (Scrub Typhus, S. pneumoniae and H. influenzae has major implications for the management and outcome of AES cases in the country with respect to mortality and morbidity. The demonstration of dengue virus emerging as an important cause of AES in the country also has significant public health impact on the National Health Program. Above all, the system of surveillance established in this project through a tiered network of laboratories is ideally poised for exploitation in the larger Global Health Security Agenda of prevent, detect and respond to infectious disease threats in the future.

\section{Longevity of immunological memory to hepatitis E virus}

\section{Anuradha Tripathy}

Email ID for Correspondence: anuradhastripathy@hotmail.com ICMR-National Institute of Virology, Pune-411001

Hepatitis E caused by hepatitis E virus (HEV) is a worldwide disease. HEV infection mostly presents as outbreaks and sporadic cases with self-limiting hepatitis, but may lead to fulminant hepatic failure, especially in pregnant women. It is estimated that one third of the world's population is infected with HEV. HEV infections can be prevented by reducing exposure to the virus and/or inducing immunity through vaccination. Whether HEV infection elicits long lasting immunity is a big question. The correlates of protection are not defined in hepatitis E. HEV specific antibody responses following exposure to HEV infection suggested antibody as one of the protective correlates and $\mathrm{T}$ cell responses have been associated with the control of hepatitis E. An in depth attempt to envisage whether natural infection with HEV confers long lasting immunity against re-infection, exhibited persistence of anti-HEV antibodies, detection of antibody secreting plasma cells (ASCs) and participation of memory $\mathrm{T}$ cells up to 26 years post recovery. There is a need of a vaccine that would protect the high risk groups from infection. A vaccine against $\mathrm{HEV}$ is not available worldwide. As a part of vaccine development, we evaluated different preparations and identified liposome encapsulated recombinant neutralizing epitope protein of HEV (rNEp) as the vaccine candidate. This candidate was shown to be immunogenic and yielded sterilizing immunity in rhesus monkeys. In an attempt to link these observations with immune parameters, immune response was assessed in mice post immunization with the vaccine candidate. The vaccine candidate effectively generated persistent and anamnestic antibody response and triggered memory B cells to differentiate into ASCs upon boosting. The presentation would elaborate on the above approaches and findings that could be useful to explore the longevity of HEV-specific memory response in future HEV vaccine trials.
Evolutionary dynamics of the 2009 pandemic influenza A H1N1 virus by viral genome and protein structure modeling analyses

\section{Sarah Cherian}

Email ID for Correspondence: cherian.ss@niv.co.in ICMR - National Institute of Virology, Pune 411001

The pandemic influenza A H1N1 (pH1N1) virus that emerged in 2009 subsequently showed multiple waves of activity globally. The evolutionary dynamics of influenza $\mathrm{A}$ viruses arise from a complex combination of mutations in the gene segments, mainly those coding for the envelope glycoproteins hemagglutinin (HA) and neuraminidase (NA). The viral diversity is further modulated by natural selection, global patterns of virus circulation and host population biology. Understanding the evolutionary dynamics of the pH1N1 virus lineages during their initial emergence and establishment in the human population have thus been the subject of several recent studies. The real-time monitoring of virus genome diversity has several applications. Phylogenetic and molecular clock analyses based on HA or whole genome sequences estimate divergence times and represent transmissions that persist within a country between different waves. The population dynamics of the $\mathrm{pH} 1 \mathrm{~N} 1$ viruses studied through Bayesian skyline plots correspond to the identification of newly evolved sub-clades and reflect the emergence of mutations in the HA antigenic sites having implications to $\mathrm{H} 1 \mathrm{~N} 1$ vaccine strain selection. The detection of marker mutations in the NA that could confer Oseltamivir drug resistance as well as functional significance of mutations getting established in other genes through protein modeling, ligand docking and molecular dynamics simulation studies can throw light on the mechanism of adaptation of the viral strains in the population. This presentation would focus on such studies undertaken in our laboratory that demonstrate how advanced phylogenetic and protein structure modeling analyses provide insights on the evolutionary aspects of the 2009 pH1N1 virus. Further, the understanding of the molecular mechanism involved in host-virus interactions would be useful during the design of the enzyme-specific inhibitors to combat influenza infections.

\section{HPV vaccine: two in one benefit}

\section{Priya Abraham}

Email ID for Correspondence: priyaabraham@cmcvellore.ac.in Department of Clinical Virology, CMC Vellore

There are over 100 different genotypes of human papilloma viruses (HPV) identified. Among the HPV genotypes that infect the anogenital region, there are around 14 oncogenic HPVs. Other genotypes are implicated in causing warts and other cutaneous lesions. The HPV vaccine has been shown to protect against cervical intraepithelial lesions and cervical cancer. However, the current vaccines against HPV protect not just against cervical cancers. It has been shown to protect against orophayngeal cancers, anal cancer and also possibly against penile, vulvar and vaginal cancers. Further, the current quadrivalent vaccines and nonavalent vaccines also protect against disfiguring genital warts which are caused by the non-oncogenic strains of HPV 6 and 11. Children born to vaccinated mothers are protected from early childhood lesions such as benign laryngeal papillomatosis. Hence, based on these findings, HPV vaccine delivers more than the well known benefit of prevention of cervical cancer. The vaccine is now recommended for both genders. Studies in India are needed to demonstrate many of the several benefits of these vaccines. Though originally recommended as a 3-dose course, the WHO currently recommends a 2 - dose course, thereby bringing down 
the cost further. It is hoped that Indigenous HPV vaccines will further reduce vaccine costs and make universal vaccination in India a reality in the not so distant future.

Host innate immune responses in alphavirus pathogenesis: insights into potential therapeutic solutions

\section{Zaid A, Chen W, Sheng KC, Taylor A, Cooper MA, King NCJ,} Ng LFP and Mahalingam S

Email ID for Correspondence: a.zaid@griffith.edu.au Emerging Viruses and Inflammation Research Group, Institute for Glycomics, Griffith University, Queensland, Australia

Alphaviruses are arthropod-borne viruses that include the arthritogenic Chikungunya and Ross River viruses, and for part of a group of emerging pathogens that is causing significant concern worldwide. From explosive outbreaks to localized epidemics, arthritogenic alphaviruses can cause debilitating arthritic disease, accompanied by fever, myalgia and cutaneous rash. There are currently no vaccines for alphaviruses or specific treatments for the inflammatory disease they cause, and recent outbreaks in the Caribbean, Indian Ocean and South-East Asia are a stark reminder of the dire need for prompt therapeutic advances. Experimental animal models have helped unravel some of the key mechanisms at play in inflammatory responses that lead to disease. Specifically, mouse models of CHIKV and RRV disease have shown how aberrant innate immune responses contribute to further inflammation, leading to tissue damage. Here, we will discuss some findings in our laboratory that bring to light the role of innate immune response mechanisms that contribute to alphavirusinduced musculoskeletal inflammation, and show the therapeutic approaches that could be employed to treat patients in the future.

\section{Insight of Zika virus infection in neural cell: the route to brain}

Mutso M, St John JA, Burt FJ, Zaid A, Žusinaite E, Merits A, Ling ZL, Grau GE, Hueston L, King NJC, Ekberg J, Mahalingam S

Email ID for Correspondence: m.mutso@griffith.edu.au Emerging Viruses and Inflammation Research Group, Institute for Glycomics, Griffith University, Queensland, Australia

Zika virus (ZIKV) has recently emerged as an important human pathogen due to the strong evidence that it causes disease of the central nervous system, particularly microcephaly and Guillain-Barré Syndrome. The pathogenesis of disease including mechanisms of neuroinvasion and cellular responses to infection are poorly understood. We characterises the in vitro ZIKV infection of a variety of neural (neuroglial) cells lines, including primary mouse and human olfactory ensheathing cells (OECs) and Schwann cells (SCs), human and mouse OEC immortalized cell lines, (hOECs and mOECs, respectively) and a human brain endothelial cell line (D3 cells). Readouts included infection kinetics, intracellular virus localisation and the host cell response. Although not as high as in non-neural cells, viral titres exceeded $10^{4} \mathrm{PFU} / \mathrm{ml}$ in all neuroglial cell types except hOEC. Despite these substantial titres, only a small proportion of neuroglial cells were infected. Intracellular staining of infected cells revealed localisation of the ZIKV NS3 protein in the cytoplasm and of NS5 primarily in the nucleus. Taken together, these results provide basic insights into ZIKV infection of a variety of neuroglial cells and will form the basis for further study of ZIKV disease mechanisms and the route to the brain.

Mutation of the N-terminal region of chikungunya virus capsid protein: implications for vaccine design

Adam Taylor, Liu X, Zaid A, Goh LY, Hobson-Peters J, Hall R A, Merits, A, Mahalingam S

Email ID for Correspondence: a.taylor1@griffith.edu.au Emerging Viruses and Inflammation Research Group, Institute for Glycomics, Griffith University, Queensland, Australia

Mosquito-transmitted chikungunya virus (CHIKV) is an arthritogenic alphavirus of the Togaviridae family responsible for frequent outbreaks of arthritic disease in humans. Capsid protein, a structural protein encoded by the CHIKV RNA genome, is able to translocate to the host cell nucleolus. In encephalitic alphaviruses, nuclear translocation induces host cell transcriptional shutoff; however, the role of capsid protein nucleolar localization in arthritogenic alphaviruses remains unclear. Using recombinant enhanced green fluorescent protein (EGFP)-tagged expression constructs and CHIKV infectious clones, we describe a nucleolar localization sequence (NoLS) in the $\mathrm{N}$-terminal region of capsid protein, previously uncharacterized in CHIKV. Mutation of the NoLS by site-directed mutagenesis reduced efficiency of nuclear import of CHIKV capsid protein. In the virus, mutation of the capsid protein NoLS (CHIKV-NoLS) attenuated replication in mammalian and mosquito cells, producing a smallplaque phenotype. Attenuation of CHIKV-NoLS is likely due to disruption of the viral replication cycle downstream of viral RNA synthesis. In mice, CHIKV-NoLS infection caused no disease signs compared to wild-type CHIKV (CHIKV-WT)-infected mice; lack of disease signs correlated with significantly reduced viremia and decreased expression of proinflammatory factors. Mice immunized with CHIKV-NoLS, challenged with CHIKV-WT at 30 days postimmunization, develop no disease signs and no detectable viremia. Serum from CHIKV-NoLS-immunized mice is able to efficiently neutralize CHIKV infection in vitro. Additionally, CHIKV-NoLSimmunized mice challenged with the related alphavirus Ross River virus showed reduced early and peak viremia postchallenge, indicating a cross-protective effect. The high degree of CHIKV-NoLS attenuation may improve CHIKV antiviral and rational vaccine design.

Computational resource for phylogenomics and therapeutic analysis of Zika virus

\section{Manoj Kumar}

Email ID for Correspondence: manojk@imtech.res.in Virology Discovery Unit and Bioinformatics Centre, Institute of Microbial Technology, Council of Scientific and Industrial Research (CSIR), Sector 39-A, Chandigarh-160036, India

Zika virus (ZIKV) is a flavivirus of Flaviviridae family and has a single-stranded positive sense RNA genome of about $\sim 11 \mathrm{~kb}$. It was responsible for recent epidemic across Africa, Southeast Asia, and in pacific islands as reported by World Health Organization in early 2016 (http://www.who.int/csr/disease/zika/en/). ZIKV is a mosquitoborne arbovirus transmitted through monkeys and Aedes mosquitoes with humans as occasional hosts. It causes a self-limiting illness with 
fever, headache, rashes, malaise, chills, etc. but may also be associated with congenital anomalies such as Guillain-Barre syndrome and microcephaly in humans. To date there is no approved vaccine or drug to prevent Zika infection and the conventional therapeutics development strategies are tedious and time consuming. Thus, in silico approaches facilitate well-timed systematic understanding of virus biology and are beneficial in revealing putative vaccine and drug candidates. In this endeavor, we have carried out in-depth computational analyses and developed a web-based multi-omics platform, ZikaVR (http://bioinfo.imtech.res.in/manojk/zikavr/) (Scientific Report, 2016). It is devoted towards ZIKV therapeutics, comparative genomics and provides essential tools for further exploration. It integrates whole genome alignments, phylogeny, genomic rearrangement, synteny, $\mathrm{CpG}$ islands, codon context and usage bias, proteins 3D structures, glycosylation sites, diagnostics primers for evolutionary analysis and diagnostics. Based on intensive computational analysis, we have proposed several potential vaccine epitope candidates. Similarly, siRNAs, miRNAs, sgRNAs and repurposed drugs were also advocated. It is anticipated that a unique blend of analyses in ZikaVR will be helpful for evolutionary, diagnostics and therapeutic aspects of ZIKVs.

Human papillomavirus derived novel cell penetrating peptide in conjugation with oligoarginine peptide for intracellular delivery in normal and cancerous cells

Shikha Saxena ${ }^{1}$, Amit Ranjan Sahoo ${ }^{1}$, Sajad Ahmed Wani ${ }^{1}$, Aruna Pandey ${ }^{1}$, Deepika Bisht ${ }^{1}$, Basavaraj Sajjanar ${ }^{1}$, Aditya Prasad Sahoo', Gandham Ravi Kumar', Satish Kumar², Pramod W. Ramteke ${ }^{4}$, Ashok Kumar Tiwari ${ }^{*}$

Email ID for Correspondence: saxena.shikha96@gmail.com ${ }^{1}$ Division of Veterinary Biotechnology, ICAR-IVRI, Izatnagar, Bareilly 243122; ${ }^{2}$ C.I.F. Bioengineering Lab, Division of Veterinary Biotechnology, ICAR-IVRI, Izatnagar, Bareilly 243122; ${ }^{3}$ Division of Standardization, ICAR-IVRI, Izatnagar, Bareilly-243122; ${ }^{4}$ Sam

Higgim Bottom University of Agriculture, Science and Technology, Allahabad, U.P

Cell-penetrating peptides (CPPs) are short cationic amphipathic molecules capable of evading cell membrane barriers. There is a pressing need for innovative therapeutic strategies to address delivery of drugs and DNA across the cell membrane to thwart infections and cellular disorders. In this direction, CPPs represent novel receptor independent, non-viral delivery systems for a large variety of cargo molecules including $\operatorname{drug}(\mathrm{s})$ and therapeutic DNA. In the present study, we have identified a novel CPP from L1 protein of human papillomavirus (HPV) capable of transduction into cells. Spatial analysis revealed that nona-arginine (R9) motif when conjugated to HPV peptide (Pep HPV-R9) doubled the internalization efficacy in $\mathrm{HeLa}$ as well as Vero cells. Time lapse images in confocal microscopy suggested that half time for internalization of Pep HPV-R9 was reduced in comparison to Pep HPV. Interaction of cargo DNA with delivery peptide, essential for internalization, was studied using circular dichroic (CD) spectroscopy and extrinsic fluorescence. Notably, the Pep HPV-R9 peptide protected DNA from enzymatic degradation and facilitated delivery of functional plasmid DNA in the cultured cells. In addition, mechanistic studies revealed that the pep HPV-R9 complex mediated cell entry was through energy independent endocytotic pathway. Furthermore, pep HPV-R9 and pep HPV-R9/DNA complexes were devoid of cytotoxicity to cells at concentrations used in transfection experiments. These findings suggest that pep HPV-R9 can serve as a novel vehicle, ideal for cell based assays and delivery of therapeutic nucleic acids.
Dengue virus circulating serotypes: trends over two decades at a tertiary care centre in New Delhi

\section{Aashish Choudhary, Megha Brijwal, Rojaleen Das, Lalit Dar}

Email ID for Correspondence: aashishpath@yahoo.co.in Virology Section, Department of Microbiology, All India Institute of Medical Sciences (AIIMS), New Delhi, India-110029

Dengue is geographically the most widespread arthropod-borne disease globally, and dengue hemorrhagic fever (DHF) holds the distinction of being the most important viral hemorrhagic fever affecting human health. Serotyping of dengue viruses (labelled as DENV 1 through 4) is important, in not only ascertaining the epidemiology of the disease, but also in predicting the possible occurrence of DHF in a community, since most cases occur in patients with secondary dengue infections. This study is a retrospective analysis to determine the circulating serotypes of DENV in Delhi by two techniques: virus isolation in cell culture, and reverse transcription PCR. This activity has been performed in our cell culture laboratory on a regular basis since 1996. Representative serum samples of patients with suspected dengue with duration of fever $<5$ days were inoculated onto the C6/36 clone of Aedes albopictus cells, and the isolates were identified by indirect immunofluorescence using serotype-specific monoclonal antibodies. Reverse transcription PCR amplifying the envelope gene was also used in selected samples to correlate the results with the other technique. The predominant serotypes identified during these 22 years were DENV 1 (1997-2004), followed by DENV 2 (2005-2007), DENV 1 (2008-2011), DENV 2 (2011-2015), and DENV 3 in 2016 and 2017. Delhi became hyper-endemic for dengue in 2003, with cocirculation of all the 4 serotypes that year. The study shows that the prevailing serotype usually remains for $1-3$ years, to be replaced by a new serotype subsequently. This likely indicates a dynamic interaction between the virus and neutralizing antibodies in the majority of the existing population, with the introduction of a new serotype in the vulnerable population. This information is now even more significant since the recent approval and use of a dengue vaccine in some parts of the world.

Investigating the molecular details of hepatitis $\mathrm{C}$ and enteric viruses of Kolkata

Chayan Bhattacharjee, Sayantan Adhikary, Aparna Mukhopadhyay

Email ID for Correspondence: aparna.dbs@presiuniv.ac.in Department of Life Sciences, Presidency University, Kolkata

Kolkata is a city famous for street side food and people with gastroenteric problems; many due to pathogens such as viruses. Typical enteric viruses spread via contaminated food include Rotavirus, Norovirus, Hepatitis A and E. Currently there are few reports on the common strains of enteric and hepatitis viruses that are predominant in Kolkata. In order to address this issue, we have been dedicated towards screening of common street side food to isolate and identify common enteric viruses that are spread via the fecal-oral route. This is achieved by precipitation of virus particles from surface washes, RNA isolation and RT-PCR using genotype-specific primers. So far preliminary results suggest the presence of Rotavirus and Norovirus in street side salad in Kolkata. Hepatitis $\mathrm{C}$ virus, a blood-borne pathogen is the leading cause of liver disease and hepatocellular carcinoma worldwide. This RNA virus has seven genotypes and over 67 subtypes. So far molecular details about the early events of viral entry remain scanty. Using pseudoparticles, we are currently 
dissecting the molecular details of HCV entry in Huh7 cell lines, particularly its timeline and dependence on microtubules. Furthermore, computational tools have revealed about $30 \%$ differences in the amino acid and nucleotide sequences across HCV genotypes. This has led us to investigate how such changes could be affecting the biology of the virus. As genotypes 1 and 3 are believed to be predominant in Kolkata, we have concentrated on these genotypes to predict how such differences could be affecting its interaction with the entry receptor CD81. Our results indicate that binding sequences, as well as affinity, differs among genotypes. We now wish to concentrate on validating these results via experiments. This work is significant as so far development of therapeutics is based on studies from only a few genotypes.

Role of secretory leucocyte protease inhibitor in viral infections of the oral cavity: a review

\section{Vidya Ajila ${ }^{1}$, Veena Shetty ${ }^{2}$, Subhas Babu $G^{1}$ \\ Email ID for Correspondence: ajila_v@yahoo.com \\ ${ }^{1}$ Department of Oral Medicine and Radiology, A B Shetty Memorial Institute of Dental Sciences, Nitte University, Deralakatte, Mangaluru, Karnataka, India-575018; ${ }^{2}$ Department of Microbiology, Research co-ordinator NUCSReM, K S Hegde Medical Academy, Nitte University, Deralakatte, Mangaluru, Karnataka, India-575018}

Human secretory leukocyte protease inhibitor (SLPI) is an 11.7-kDa cationic protein and a member of the innate immunity-associated proteins. SLPI is considered to be a major factor in preventing HIV-1 transmission through oral secretions due to its antiretroviral action. In the presence of HSV infection, there is downregulation of SLPI which, in turn, increases the risk of HIV infection. Further, SLPI has shown an inverse correlation with HPV-positive tumours. The present review focuses on the role of SLPI in viral conditions affecting the oral cavity with emphasis on its effects on HIV, HPV and HSV.

\section{Identification of circulatory microRNAs in different phases} of hepatitis $B$ virus induced disease progression and its prognostic relevance in hepatocellular carcinoma

\section{Neeti Nadda, Shashi Paul, Anoop Saraya, Shalimar and Baibaswata Nayak}

Email ID for Correspondence: baibaswata@yahoo.com Departments of Gastroenterology and Radiodiagnosis, All India Institute of Medical Sciences, Ansari Nagar, New Delhi, India

Hepatitis B virus (HBV) infection leads to chronic liver disease, cirrhosis, and cancer. Majority of hepatocellular carcinoma (HCC) patients are diagnosed at advanced stages and often associated with poor prognosis and survival. The loco-regional therapy is the mainstay of treatment. Due to the absence of a suitable biomarker, radiological imaging techniques like DPCT or MRI are mostly used for diagnosis and monitoring therapeutic response, which is not cost effective. To identify circulatory miRNAs that are differentially expressed in treatment naïve chronic hepatitis B (CHB), cirrhotics and HCC patients. To evaluate differentially expressed miRNA for monitoring therapeutic response to locoregional therapy. Circulatory miRNA profiling of 84 miRNAs that are differentially expressed in serum was carried out using miScript miRNA PCR Array in healthy and HBV infected treatment naïve, cirrhotic and HCC patients $(n=32)$. Total RNA isolated from serum were polyadenylated, reverse transcribed and amplified by real time PCR and differential miRNA array data was analyzed by comparative $\mathrm{Ct}$ method. The HCC patients of HBV etiology were staged and treated as per BCLC criteria and the therapeutic response was assessed by mRECIST criteria. The correlations of selected miRNA expressions in real time PCR in pre and post-therapy were assessed for therapeutic response. Differential expression profiling of 84 miRNAs in serum of healthy vs $\mathrm{CHB}$, cirrhotic and HCC patient has shown significant up-regulation for 2 (1.4-6.46 fold), 9 (1.02-14.28 fold) and 5 (1.40-74.54) miRNA after normalization with exogenous Cel miR39 and endogenous SNORD95. Differential expression of CHB vs HBV induced cirrhotic and HCC have shown 0 and 3 (1.02-57.08 fold) miRNAs. The miRNAs (miR-16, - 21 and - 221) upregulated in HCC were further evaluated for changes in expression during locoregional therapy at baseline and 1-month post-therapy $(n=62)$. Logistic regression analysis for prediction of the response found significance for miR-221 $(P=0.05)$ but not miRNA-21 and $-16(P=0.231)$. The miRNA$221,-21$ and-16 have potential as a biomarker for the predictor of therapeutic response.

Exploring rotavirus proteins to design $B$ and $T$ cell multi-epitope subunit vaccine using immunoinformatics approach

\section{Nima D. Namsa, Yengkhom Damayanti Devi}

Email ID for Correspondence: namsa@tezu.ernet.in Department of Molecular Biology and Biotechnology, Tezpur University, Napaam 784 028, Tezpur, Assam

Rotavirus (RV) is the leading cause of severe acute gastroenteritis in infants and young children of $<5$ years of age. Substantial evidence suggests that rotavirus vaccines provide heterotypic protection against a wide range of genotypes. However, a small difference in effectiveness against individual strains may lead to the emergence of escape strains over time making continued monitoring of circulating strains important following vaccine introduction. Therefore, a holistic approach is needed to generate $\mathrm{T}$ and $\mathrm{B}$ memory cells to mediate long-term immunity against RV infection. Consequently, a comprehensive immunoinformatics approach was applied to design rotavirus protein based multi-epitope subunit vaccine construct containing B and $\mathrm{T}$ cell epitopes. Further, the allergenicity and antigenicity were also predicted to ensure the safety and immunogenic behavior of final vaccine construct. The MHC-I and MHC-II restricted peptides identified in this study could interact with the alleles of major HLA supertypes that are known to have cumulative $95.71 \%$ population coverage for the five major ethnicities (Black, Caucasian, Hispanic, Native American, and Asian). By using in silico docking techniques the predicted epitope was further assessed for binding against HLA molecules to verify the binding cleft interaction. Based on in silico prediction, we have designed three chimeric genes (SA11-VP6-BT, SA11-VP6-7-4L and SA11-VP6-7-4B) containing the predicted Band T cell epitopes of major capsid protein VP4, VP6 and VP7 for expression and purification in E. coli system. These chimeric antigens will be used in generating in-house polyclonal antibody for possible development of ELISA based diagnostic tool for detection of rotavirus antigen in stool samples. Further, this computational study warrants the experimental validation to ensure the immunogenicity and safety profile of epitope based vaccine construct, which may be further helpful to control rotavirus infection. 
Molecular characterization of Zika virus sequences from Mexico

Rama Adiga ${ }^{1}$, Celia Boukadida ${ }^{2}$, Jesús M. Torres-Flores ${ }^{3}$, Martha Yocupicio-Monroy ${ }^{4}$, Elvira Piten-Isidro², Amarant Y. Rivero-Arrieta ${ }^{2}$, Yara A. Luna-Villalobos ${ }^{2}$, Liliane Martínez-Vargas ${ }^{2}$, Sofía L. Alcaraz-Estrada ${ }^{5}$, Klintsy J. Torres ${ }^{2}$, Rosalia Lira ${ }^{6}$, Gustavo Reyes-Terán², Edgar E. Sevilla-Reyes ${ }^{2}$

Email ID for Correspondence: rama_adiga@nitte.edu.in ${ }^{1}$ Division of Bioinformatics, Nitte University Centre for Science Education and Research, Deralakatte, Mangalore, India 575018; ${ }^{2}$ Departamento de Investigación en Enfermedades Infecciosas, Instituto Nacional de Enfermedades Respiratorias Ismael Cosío Villegas (INER), Ciudad de México, Mexico; ${ }^{3}$ Laboratorio de Virología, Escuela Nacional de Ciencias Biológicas, Instituto Politécnico Nacional, Ciudad de México, Mexico; ${ }^{4}$ Posgrado en Ciencias Genómicas, Universidad Autónoma de la Ciudad de México, Ciudad de México, Mexico; ${ }^{5}$ Centro Médico Nacional 20 de Noviembre, Instituto de Seguridad y Servicios Sociales de los Trabajadores del Estado, Ciudad de México, Mexico; ${ }^{6}$ Unidad de Investigación Médica en Enfermedades Infecciosas y Parasitarias, UMAE Hospital de Pediatría, Instituto Mexicano del Seguro Social (IMSS), Ciudad de México, Mexico)

Zika virus is an arthropod-borne flavivirus associated with congenital malformations with extreme severity and neurological complications. The devastating phenomenon spread globally causing birth defects and Guillain-Barré syndrome. The epidemic turned into a public health emergency as declared by WHO in February 2016 that lasted for 10 months. During this period Zika virus spread across the America with autochthonous transmission reported from 42 countries. The Latin American country of Colombia also reported 42 cases of Zika associated congenital syndrome followed by U.S.A. with 26 cases as on October 2016. The genetic evolution of the virus in the Americas was the objective of the study which helped to throw light on its pathogenicity. NS5 gene showed mutation of a highly conserved threonine to alanine. The hydrophilic amino acid was substituted with hydrophobic amino acid at the conserved amino acid position of 581 and was useful in genotyping the Zika virus. The classification of a Latin American genotype of Zika virus isolated from countries of Colombia, Mexico, Panama, and Martinique. The sequences analyzed were collected from the city of Oaxaca and Chiapas in Mexico where microcephaly was associated with Zika infection. The sequences were made available in public domain. The molecular phylogenetic analysis showed genetic polymorphism in the NS5 sequence from Mexico. Motif analysis and modeling the structure of NS5 by molecular replacement was carried out. Structural superimposition with wild-type virus suggested structural polymorphism. Viruses have been known to use host-like amino acid motifs to interact with host proteins as part of molecular mimicry. It is predicted that the genetic polymorphism at position 581 is one of the viral strategies to promote host modulation and facilitate viral-host interaction.

Chikungunya nsP1 directly interacts with nsP2 and modulates its ATPase activity

Sameer Kumar ${ }^{1}$, Abhishek Kumar ${ }^{1}$, Prabhudutta Mamidi ${ }^{1}$, Sagarika Mudulli ${ }^{1}$, Bharat Bhusan Subudhi ${ }^{2}$, Soma Chattopadhyay ${ }^{*}$

Email ID for Correspondence: sochat.ils@gmail.com

${ }^{1}$ Institute of Life Sciences, Nalco Square, Bhubaneswar-751023, India; ${ }^{2}$ School of Pharmaceutical Sciences, Siksha O Anusandhan University, Bhubaneswar, India

Chikungunya virus (CHIKV) is an RNA virus, which has created an alarming threat in the modern world due to unavailability of vaccine and antiviral compounds. There are four non-structural proteins (nsP1-4) of CHIKV synthesized during infection in host cells. The nsP2 contains ATPase, RTPase, helicase and protease activities, whereas, nsP1 is a viral capping enzyme. In alphaviruses, the four nsPs form the replication complex (RC) in the cytoplasmic side of the cell membrane and thus might interact with each other via RC. In this study, we characterize the interaction between $\mathrm{CHIKV} n \mathrm{nP} 1$ and $\mathrm{nsP} 2$ during infection. It was observed that, both the proteins co-localize in the cytoplasm and interact in the CHIKV infected cells by confocal microscopy and immunoprecipitation assay. Further, it was demonstrated through mutational analysis that, the amino acids 1-95 of nsP2 N-terminus and 170-288 of nsP1 membrane binding domain are responsible for their direct interaction. Additionally, it was noticed that the ATPase activity of nsP2 is enhanced significantly in the presence of nsP1, indicating the synergistic effect of nsP2 and nsP1 due to this interaction. In silico analysis showed close ( $\leq 1.7 \AA$ ) polar interaction (hydrogen bond) between Glu4, Arg7, 96, 225 of nsP2 with Lys256, 206, Val367 and Phe312 of nsP1 respectively. This investigation provides molecular characterization of CHIKV nsP1-nsP2 interaction which might be a useful target for rational designing of antiviral drugs.

Molecular characterization of hemagglutinin gene of Influenza A (H3N2) viruses circulating in India during 2014-2016

Saritha Sivadas ${ }^{1}$, Anitha $\mathbf{J}^{1}$, Sudheesh $\mathbf{N}^{1}$, Anjana $\mathbf{K}^{1}$, VahidRajabali Zeddah ${ }^{1}$, Dr. G Arunkumar ${ }^{1}$

Email ID for Correspondence: 7sarithasivadasnileshwar@gmail.com Manipal Centre for Virus Research, Manipal University

Influenza virus infection remains a major cause of morbidity and mortality worldwide. The burden can be minimized by vaccination. Current Influenza vaccines induce antibodies that target the highly variable immunodominant epitopes of the viral hemagglutinin (HA). In this study, genetic variation of HA gene of Influenza A (H3N2) virus positive samples from different parts of India during the time period of 2014-2016 was analyzed in comparison to the vaccine strains. Influenza A (H3N2) virus positive samples collected from different parts of India as a part of Acute Febrile Illness (AFI) surveillance was used in this study. A total of 22 samples were amplified by conventional PCR using primer targeting H3 gene. The amplified products were sequenced using modified Sanger Sequencing method. Sequences was aligned using Clustal Omega and Phylogenetic tree were constructed using Mega6. Analysis of antigenic site residues was performed in comparison to vaccine strains of 2014, 2015 and 2016 Influenza season. In comparison to 2014-2016 vaccine strain, antigenic site mutations (F175Y and K176T) were detected in 20 isolates. Mutations Q327H (Site C) and N137K (Site D) were detected in 20 and 12 isolates, respectively and these mutations were absent in the vaccine strains. Continuous monitoring of antigenic changes are important as the data can contribute to decision making on Influenza vaccination strategies and also track the emergence of new strains of Influenza.

Molecular characterization of hemagglutinin (HA) gene of influenza A (H1N1 and H3N2) virus

\section{Anitha J, G Arunkumar}

Email ID for Correspondence: anitha_jagadesh@yahoo.com Manipal Centre for Virus Research, Regional Reference Laboratory for Influenza Virus and ICMR Grade-I Virus Diagnostic Laboratory, Manipal University, Manipal- 576104 
Influenza is one of the major threats to human and animal health due to its high transmissibility, severe morbidity and mortality. Influenza virus alters its antigenicity by accumulating mutations on the surface glycoproteins, hemagglutinin (HA), and neuraminidase. HA is the major surface protein and studies on the genetic analysis of HA protein is important to know about the variability in circulating influenza viruses and to design vaccine. Therefore, the objective of this study was to characterize the HA gene of influenza A (H1N1 and H3N2) viruses. Influenza A virus positive samples from the year 2017 were included in the study. In order to check the rate of nucleotide/ aminoacid changes, 7 influenza A (H3N2) and 10 influenza A (H1N1) positive samples were selected by purposive sampling. Polymerase chain reaction was carried out for full length amplification of $\mathrm{H} 1$ and $\mathrm{H} 3$ genes. The amplicons were purified and sequenced. Amino acid sequences were compared with the 2017 vaccine strain sequences. H1 gene sequences showed $98.74-99.28 \%$ identity with 2017 vaccine strain A/Michigan/45/2015 (H1N1). Mutations K180Q and S202T (i.e. K166Q and S188T H1 numbering) in antigenic site of HA protein that might have caused antigenic drift resulting in a change of circulating influenza A (H1N1) in 2015 was found in all isolates. Among the mutations detected T408S, A26S and S551R were new mutations that has not been reported globally to date, but these mutations were in non-antigenic sites. H3 gene sequences showed 96.46-97.70\% identity with 2017 vaccine strain A/HongKong/4801/2014 (H3N2). Mutation K176T and P210L that might affect antibody binding was detected in all the 7 influenza A (H3N2) isolates. Amino acid substitutions in $\mathrm{H} 1$ gene sequences occurred at a slower rate in comparison to $\mathrm{H} 3$ gene sequences. Continuous monitoring of the antigenic variations in HA gene is important for recommending vaccine strain with high similarity with circulating virus.

\section{Alignment-free approach for viral typing}

\section{Urmila Kulkarni-Kale}

Email ID for Correspondence: urmila@bioinfo.net.in; urmila.kulkarni.kale@gmail.com

Bioinformatics Centre, Savitribai Phule Pune University, Pune 411007. India

Characterisation viral isolates for types and subtypes is an essential prerequisite for management, control, and prevention of infectious diseases of humans and live stocks. Currently, both, experimental and computational approaches are used for typing. The computational approaches are based on phylogenetic analyses of genomic and/or proteomic regions, which are recommended by the International Committee of Taxonomy of Viruses (ICTV) and its study groups. The most frequently used methods of phylogeny are alignment-based and are compute intensive. Typing of viruses using phylogenetic analysis is a multi-step process that needs to be repeated every time a new viral isolate is sequenced. Furthermore, uncertainties associated with sequence alignments and their implications on alignment-based phylogeny analyses are known. An alignment-free method for phylogeny, clustering, and typing has been developed in-house (1). The method is based return time distribution, a statistical concept in the field of stochastic processing. The method has been successfully applied for typing of viruses such as Mumps, Dengue, West Nile and Rhino (1-4) and the typing servers are available online. For every virus, input data may vary for the sequence of an individual gene to genome. The method and respective servers are found to work with $100 \%$ accuracy, sensitivity, and specificity. These servers require users to provide sequence(s) of the viral isolate(s) as an input. The methodological modifications to the basic RTD algorithm along with challenges in the application of the method to other viral pathogens will be discussed. The servers are expected to be useful to monitor the diversity of circulating types of viruses.

\section{Poster Presenatation}

\section{Epidemiological dynamics of dengue virus in Shivamogga,} Karnataka

\section{Abdulmajeed Jazeel, Kumar Rajemdra, Jayaram Anup, Sheik Shahin, Rao Chaitra, Govindakarnavar Arunkumar}

Email ID for Correspondence: jazeel.mcvr@manipal.edu Manipal Centre for Virus Research, Manipal University, Manipal, Karnataka, India-576104

Dengue is an acute viral infection with potential fatal complications. The epidemiology of dengue virus and its prevalent serotypes has been ever changing. All the four serotypes of the virus have been in circulation and documented from various parts of the country. In 2014 Manipal Centre for Virus Research initiated the hospital-based laboratory-supported acute febrile illness (AFI) surveillance at subdistrict level sentinel hospitals across several states to map the aetiology of AFI in general including Dengue. From June 2014 through July 2017, we identified a total of 8109 AFI cases admitted to the sentinel sites in Shivamogga district, Karnataka. Of these cases, 398 (5\%) was positive for Dengue Virus. Among the four sentinel sites, JC Hospital, Thirthahalli had 186 (47\%), Taluk Hospital, Soraba 94 (24\%), Taluk Hospital, Sagara 78 (20\%), and Taluk Hospital, Hosanagara $40(10 \%)$ cases were positive for Dengue. The median age was 25 years (IQR 18-35), 257 (65\%) were males. The cases had complaints of myalgia (84\%), headache $(81 \%)$, general weakness $(74 \%)$, arthralgia $(57 \%)$, vomiting $(44 \%)$, retro orbital pain $(29 \%)$, cough (39\%) and rash (2\%). Around $48 \%$ had platelet counts below 1.5 Lakhs cells $/ \mathrm{mm}^{3}$. All the four serotypes of Dengue virus were prevalent in the district; DENV-1 (48\%), DENV-3 (26\%), DENV-2 $(22 \%)$ and DENV-4 (4\%). Dengue was prevalent in the district throughout the year with major peaks observed during the months of May- June of 2015 and 2016. This study describes the occurrence of Dengue in Shivamogga district of Karnataka and its characteristics. Real-time laboratory supported disease surveillance will aid in early detection of cases, and provide specific insights to the epidemiology and dynamics of the disease, which will act as a strong baseline for planning effective public health measures for control of the disease.

\section{Comparative genomics of flaviviruses to identify conserved drug} targets

\section{Akanksha Rajput and Manoj Kumar}

Email ID for Correspondence: akanksha@imtech.res.in Virology Discovery Unit and Bioinformatics Centre, Institute of Microbial Technology, Council of Scientific and Industrial Research (CSIR), Sector 39-A, Chandigarh-160036, India

Flaviviruses genus has arboviruses that are enveloped and posititve sense, single stranded RNA viruses. Geographically they are confined to tropical and sub-tropical regions, mainly in Southeast Asia and Africa. This genus consists of dengue virus (DENV), Japanese encephalitis virus (JEV), West Nile virus (WNV), Zika virus (ZIKV), yellow fever virus (YFV), etc. These viruses cause serious outbeaks/ epidemics globally and lack specific therapeutics. Therefore, there is a need to accelerate efforts towards discovery of drugs and computational approach becomes highly useful in identifying potential candidates. In this study, we employed various comparative genomics approaches to decipher the potential conserved drug targets. We used reference genomes of flaviviruses along with Indian strains and carried out the whole genome comparative alignment using progressive MAUVE, GenomeRing; phylogeny by MEGA7.0 and recombinant 
analysis by RDP4 package. Further, protein sequence and structure alignment of RNA dependent RNA polymerses (RdRP), one of the consensus and potential drug target, was done by Clustal Omega, LSQMAN and PyMol. The synteny analysis identified a few conserved regions with varied patterns. These conserved regions were within the genomes namely structural (core, premembrane, envelop) and non-structural (NS1-NS5) regions. The phylogenetic analyses grouped them into major clades i.e. ZIKV and Tembusu virus; JEV and Spondweni virus; DENV1-4 with Uganda S virus, etc. with good bootstrap support. Further, a few recombination events were also found among the flaviviruses e.g. YFV, Entebbe bat virus and Sokoluk virus; JEV, Murray valley encephalitis virus and Chaoyang virus. One of conserved block derived from comparative genomics approach is RdRP which catalyzes RNA replication from RNA template. The percent identity of RdRP protein varied from $\sim 60$ to $80 \%$ among reference strains. Moreover, the 3D alignment revealed that they are structurally more conserved at key residues along with Indian strains. This comparative genomics analyses is helpful to decipher potential drug targets against viruses.

\section{Coxsackievirus A6 (CV-A6) encephalomyelitis: a case report}

Aswathyraj S ${ }^{1}$, Sasidharanpillai Sabeena ${ }^{1}$, Kamalakshi G Bhat ${ }^{2}$, Kiranchandrabharani $^{1}$, Govindakarnavar Arunkumar ${ }^{1}$

Email ID for Correspondence: aswathyraj2587@gmail.com ${ }^{1}$ Department of Virus Research, Manipal University, Karnataka Manipal, India-576104; ${ }^{2}$ Department of Paediatrics, Kasturba Medical College, Mangalore

Coxsackievirus A6 (CV-A6) has recently emerged as the predominant circulating enterovirus strain causing Hand Foot and Mouth Disease (HFMD) worldwide. CV-A6 is a single stranded RNA virus; belonging to the family Picornaviridae and genus Enterovirus. Enteroviruses such as EV71 and CVA16 are more often associated with neurological manifestations mainly among children under 5 years of age. Here we report a case of Coxsackievirus A6 (CV-A6) associated encephalomyelitis in a 4-year old pre-school child from North Kerala. The child was brought to emergency department of Paediatrics, Kasturba Medical College, Mangalore when he developed altered sensorium after 3 days of fever, coryza, conjunctival congestion and myalgia. The Cerebrospinal fluid (CSF) was tested positive for Pan-enterovirus by real-time PCR. A conventional RTPCR using specific primers targeting CV-A6 Viral Protein 1 (VP1) gene was used for genotyping. Usually, EV71 and Coxsackievirus A16 are the main neurotropic enteroviruses and the current vaccine research is focussing on these subtypes. Though CVA6 is commonly present in hand foot, mouth disease in the paediatric age group, there are reports of CVA6 associated encephalomyelitis from China. Laboratory-based surveillance is essential to identify the currently circulating strains and based on the epidemiological information, multivalent enterovirus vaccines should be develop.

Improved Japanese encephalitis virus vaccine using recombinant Newcastle disease virus as a vector

\section{Barnali Nath, Sachin Kumar}

Email ID for Correspondence: barnalinath2014@gmail.com Department of Biosciences and Bioengineering, Indian Institute of Technology Guwahati, Guwahati, Assam 781039, India

Japanese encephalitis (JE) is a mosquito-borne viral disease. It is a global public health concern since it causes an acute encephalitis syndrome (AES). A large number of JE/AES cases are reported to occur in areas with established or developing JE vaccination program. Partial vaccine coverage and emergence of new variants of Japanese encephalitis virus (JEV) might be playing an important role. Newcastle disease virus (NDV) is known to be an attractive vaccine vector candidate for both human and animal pathogens. In the present study, a recombinant NDV was generated entirely from cloned cDNA. Furthermore, the recombinant NDV was used to express the envelope protein (E) of JEV. The recombinant NDV expressing E protein of JEV was characterized in cell culture, embryonated chicken eggs and 1-day-old chickens. The recovered recombinant virus could be a potential tool for the development of diagnostics and vaccine against JEV infection. We are trying to explore the possibility of using mice as a model to study the immunogenicity of the recombinant vaccine.

\section{Human metapneumovirus (hMPV) in acute respiratory illness cases-India}

Chandra Bharani Kiran, Devadiga Santhosha, Rao Chaitra, Jayaram Anup, Sushama Ashwathyraj, Govindakarnavar Arunkumar

Email ID for Correspondence: kiran.chandra@manipal.edu Manipal Centre for Virus Research, Manipal University, Manipal, Karnataka, India-576104

Human metapneumovirus (hMPV) can cause upper and lower respiratory disease in people of all ages, especially among young children, older adults, and people with weakened immune system. Human metapneumovirus has been recently identified as the second leading viral cause of paediatric acute respiratory tract infections (ARTIs) after RSV. Human metapneumovirus (hMPV) is a single negativestranded RNA-enveloped virus classified in the Pneumovirinae subfamily of the Paramyxoviridae family. In 2014 Manipal Centre for Virus Research initiated the hospital-based acute febrile illness (AFI) surveillance at District and sub-district level sentinel hospitals across several states to map the aetiology of AFI cases. A total number of 27,586 acute febrile illness cases were enrolled in the surveillance during June 2014 to July 2017, of which 18,415 cases had acute respiratory tract illness. Out of these ARTI cases, $70(0.4 \%)$ were positive for human metapneumovirus. The median age of the confirmed cases was 9 (IQR 5-25.5), though majority of the cases were children below 18 years of age $(48,68.5 \%)$. Sex ratio was $1: 1.06$. The confirmed cases had clinical symptoms such as cough (90\%), coryza $(63 \%)$, sorethroat $(37 \%)$, breathlessness $(12.9 \%)$, myalgia $(57.1 \%)$, nausea $(24.3 \%)$, vomiting $(25.7 \%)$. Human metapneumovirus seen throughout the year with peak activity during November to March. Thoughthe prevalence observed to be less from the study; it gave insight into the role of human metapneumovirus in ARTIs among children. The study details from the baseline data for further research on epidemiology and disease burden of human metapneumovirus in India.

\section{Binding of CD81 varies among HCV genotypes: an in silico} prediction

Chayan Bhattacharjee ${ }^{1}$, Suman Nandy ${ }^{2,3}$, Aparna Mukhoapdhyay ${ }^{1}$

Email ID for Correspondence: chayan.rs@presiuniv.ac.in ${ }^{1}$ Department of Life Sciences, Presidency University, Kolkata; ${ }^{2}$ Bioinformatics Infrastructure Facility, North-Eastern Hill University (NEHU), Tura Campus, Tura Meghalaya; ${ }^{3}$ Department of 
Biochemistry and Biophysics, University of Kalyani, Kalyani, West Bengal

Hepatitis $\mathrm{C}$ virus (HCV) infection is a leading cause for chronic liver disease and hepatocellular carcinoma. As a single stranded RNA virus, HCV shows a high level of genetic diversity which results in viral quasispecies and evolution into 7 different $\mathrm{HCV}$ genotypes and more than 67 subtypes. Genotypes 3 and 1, however, have been found to be the predominant genotypes in the Indian sub-continent. Viral entry is via different hepato-cellular receptors, especially tetraspanin CD81. Interaction between CD81 and the viral glycoprotein E2 is followed by clathrin mediated endocytosis in a time dependent manner. In our present work using HCV pseudoparticles, we investigated the time line of HCV entry. Furthermore, the structural and functional differences between genotypes 1 and 3 were analysed using computational tools. The sequential diversity of the surface glycoproteins of HCV genotypes $1 \mathrm{a}, 1 \mathrm{~b}, 3 \mathrm{a}$ and $3 \mathrm{~b}$ were studied at nuclear and protein level. Due to unavailability of appropriate template structures in Protein Data Bank, the 3D structure of the viral and the receptor proteins were modelled by template-based modelling using remote template, fold recognition, and ab initio approaches. In order to predict the binding site of E2, structure and sequence based prediction servers were utilized. ZDOCK, the rigid body docking algorithm, was employed dually, once to predict binding interface residues of respective E2 $\mathrm{s}$ against the known binding residues of CD81 and later to model the final HCV E2-CD81 complexes with the binding sites of both proteins. Our results indicated a clear difference in the binding region among the $\mathrm{HCV}$ glycoproteins as well as distinct differences in binding affinity towards CD81. This study suggests that the wide sequence variation observed among HCV genotypes has a predicted outcome in HCV's interaction with its entry receptor. This when verified with wet lab, could have implications in the development of therapies and disease management.

\section{Hospital-based surveillance of rotavirus diarrhea among under- five children in RIMS, Imphal, Manipur}

\section{Damayanti Yengkhom ${ }^{1}$, Mimi Adhikary ${ }^{1}$, Shyamsunder Singh ${ }^{2}$, Nima D. Namsa}

Email ID for Correspondence: dama23@tezu.ernet.in

${ }^{1}$ Department of Molecular Biology and Biotechnology, Tezpur University, Napaam 784 028, Tezpur, Assam; ${ }^{2}$ Department of Pediatrics, Regional Institute of Medical Sciences (RIMS), Imphal 795 004, Manipur

Rotavirus infection is the common cause of severe diarrhea among under five children. This study was undertaken with an objective to carry out a hospital-based surveillance of rotavirus gastroenteritis among under-five children to estimate the disease burden and to identify the prevalent strains of rotavirus in the population under surveillance in Manipur, North-East India. The study was conducted in both outpatient and indoor patient admitted with acute gastroenteritis during October 2015 to June 2016 at the Regional Institute of Medical Sciences (RIMS), Imphal. Of the total 204 stool samples collected, 144 (71.0\%) samples were tested positive for rotavirus VP6 antigen using ELISA. VP4 and VP7 genes from a subset of samples were sequenced for phylogenetic analysis. A peak increase in the occurrence of rotavirus-led diarrhoea was observed mainly in the winter months during the study period. Of the 144 rotavirus-positive stool samples, 117 samples could be genotyped based on sequencing of VP7 gene. The findings of this study revealed the presence of commonly detected $\mathrm{G}$ genotypes such as $\mathrm{G} 1$ (45.8\%), G9 (30.2\%), G3 $(25.0 \%), \mathrm{G} 2(13.54 \%), \mathrm{G} 4(9.37 \%)$ and G12 (1.04\%). The most common $\mathrm{P}$ genotypes found in the clinical samples were $\mathrm{P}[8], \mathrm{P}[6]$ and P[4]. In conclusions, the present surveillance study shows the high prevalence of rotavirus-led diarrhoea among under five children's visiting RIMS, Imphal and this hospital-based surveillance study had relatively a smaller sample size, hence the results may not give the true picture of the disease burden in the community. This study also highlights the most commonly circulating rotavirus strains and emerging G12 strains in this region and the need for continuous monitoring of rotavirus strain evolution during post-rotavirus vaccination.

Computational docking studies of the 2009 pandemic influenza A H1N1 virus polymerase acidic (PA) endonuclease as an antiviral target

\section{Dipali Bhoye, Megha Agrawal, Abhisek Behera, Dev Jha, Sarah S. Cherian}

Email ID for Correspondence: d.bhoye24@gmail.com Bioinformatics Group, National Institute of Virology, Pune, India 411001

With the continued global circulation and evolution of the 2009 pandemic influenza A H1N1 (pH1N1) virus, the need for novel antiviral drugs which are less prone to resistance becomes obvious. The influenza polymerase acidic (PA) endonuclease, which is a dinuclear metalloenzyme that plays a crucial role in initiating virus replication, is one such promising target and several efforts are being made to develop small-molecule inhibitors against this polymerase subunit. The structure-based development of inhibitors that target PA endonuclease is now possible with the recent crystal structure of the 2009 pandemic influenza A H1N1 (pH1N1) virus PA catalytic domain. In this study, we sought to understand the molecular mechanism of inhibition by several derivatives of $\mathrm{C}_{60}$ fullerene compounds that were reported to have good anti-influenza activity experimentally. Computational docking simulations were performed of different fullerene $\left(\mathrm{C}_{60}\right)$ derivatives with $\mathrm{PA}$ endonuclease domain of $\mathrm{pH} 1 \mathrm{~N} 1$ Influenza A virus (PDB ID: 4LN7) using AutoDock Vina, an automated module of PyRx software. The interactions of PA with fullerene were compared with the interactions of $\mathrm{PA}$ with its mononucleotide substrate (PDB ID: 4AWH) using Discovery studio 2017 client. Docking simulation studies, suggested that fullerene derivatives bind into the active pocket of PA endonuclease with good occupancy. The fullerene derivatives bind with better binding affinity as compared to the basic fullerene structure $\left(\mathrm{C}_{60}\right)$ in terms of additional non-covalent and strong pi-interactions. The most conserved interaction, which was observed among all fullerene derivatives, is between amino acid Tyr24 and the closest aromatic ring of the fullerene structures. This implies that fullerene structures could block access to the active site region and hence could be very good competitive inhibitors over the natural ligands of the PA protein, thus demonstrating the possibility of developing fullerene derivatives into novel anti-influenza A drugs in the future.

\section{Essential oils: a medicated gargle against H1N1}

\section{Sweta Kothari, Pavankumar Todkar, Durga Bethala, Surabhi Rode}

Email ID for Correspondence: durga.bethala@gmail.com Haffkine Institute for Training, Research and Testing, Parel, Mumbai $-400012$

pdm 09 H1N1 Influenza A is a continuously evolving form of viral infection, prevalent in South Eastern Asia, and a rise in morbidity and 
mortality has been observed inspite of presence of a vaccine regimen. There arises a need to develop a safe, affordable, and potent anti-viral agent against pdm 09 Influenza A H1N1. Some essential oils have demonstrated effective antimicrobial and antiviral properties in experimental conditions. However, most of these studies tested the liquid oil phases, which are generally less practical and are potentially toxic for oral applications. With this rationale thyme, tea tree, lemon and lavender essential oils were made into stable formulations in aqueous medium. In order to target the upper respiratory tract a potent $\mathrm{o} / \mathrm{w}$ (oil in water) emulsion based gargle formulation complied under British Pharmaceutical Codex (BPC) was developed by homogenization. In vitro cytotoxicity analysis of essential oils were carried out on MDCK cell line using MTT assay and virucidal activity was determined by amido black staining. CC50 values of the formulation of lemon oil, lavender oil, thyme and tea tree oil was found to be $0.063,0.03,0.015$ and $0.007 \% \mathrm{v} / \mathrm{v}$ respectively. The reduction of viral infectivity by these essential oils was measured by identifying the reduction in cytopathic effects using Spearman Karber method. A comparative study of antiviral activity of these emulsion based gargle formulations against pdm 09 Influenza A H1N1 was evaluated on real-time PCR. From the present study, it could be concluded that lemon oil and lavender oil based gargles had a higher log reduction values 2.37 and 2.34 respectively, than thyme oil and tea tree based gargles showing 1.67 and $1.63 \log$ reduction respectively. Our study lays the foundation for larger and more intensive pharmaceutical studies in order to develop a target specific gargle formulation.

Sentinel influenza surveillance among inpatient cases in a district level hospital in Kerala, India from 2015 to 2017

\section{Jayaram Anup, Abdulmajeed Jazeel, S Robin, Devadiga Santhosh, Chaitra Rao, Sushama Aswathyraj, Govindakarnavar Arunkumar}

Email ID for Correspondence: anup.jayaram@manipal.edu Manipal Center for Virus Research, Manipal University, Manipal, Karnataka, India-576104

Influenza virus is a moving target causing severe illness in approximately 3-5 million cases and 2,50,000-5,00,000 deaths annually in the world. Though India has an Influenza surveillance programme, data generated is scanty and mostly urban based. Here we describe the findings from a hospital-based active surveillance of Acute Febrile Illness in a district level hospital in southIndia. As part of the Hospital-Based Surveillance of Acute Febrile Illness (AFI) in India study under Global Health Security (GHSA), we had established a sentinels it in District Hospital Mananthawady, Wayanad district, Kerala in June2015. All admitted cases off ever with $<15$ days duration were recruited. All the cases satisfying SARI case definition was included for this analysis. The throat/nasal swabs collected from SARI cases were tested for Influenza viruses A (H1N1) pdm09 and Influenza A (H3N2) and Influenza B using Real Time-PCR. Data was analyzed on demographic characteristics, mortality, and seasonality. From June 2015 through July 2017, we have recruited 2372 AFI cases including 1242 (52\%) SARI cases. Of that 319 (25.7\%) tested positive for Influenza Viruses. While $153(48 \%)$ were positive for Influenza A (H1N1) pdm09, 89 (28\%) were Influenza A (H3N2) and 77 (24\%) Influenza B virus. The median age and the inter-quartile range was 34 (21-48), with little variation among the three viruses. $174(55 \%)$ were females. Their clinical spectrum included coryza (59\%), sore throat $(44 \%)$, vomiting $(25 \%)$, breathlessness $(11 \%)$, myalgia $(11 \%)$ and diarrhoea $(1 \%)$. Around $8(1 \%)$ were referred to tertiary care hospitals. No deaths were reported during this study period. A major variation in the seasonality was noticed among the viruses. The activity of Influenza A (H1N1) pdm09 peaked during June-
September in 2015 while 2017 had a distinct summer (February to April) peak. However, activity of Influenza A (H3N2) peaked in varied months of May to December in the year 2015 and 2017. The Influenza B virus was predominant from August-November in 2015 and 2016. This study documents that influenza is prevalent throughout the year. There was no mortality associated with Influenza reported in this hospital. Early case detection and prompt case management might have contributed to the zero mortality.

Molecular detection of alphaviruses in acute febrile illness cases in south west India: a retrospective study

Josmi Joseph, Santhosha Devadiga, Aswathyraj S, Rajendra Kumar M G, Sanjay E R, Sabeena S, Shilpa C, Kavitha K, Sudheesh N, Govindakarnavar Arunkumar

Email ID for Correspondence: josmimariyett91@gmail.com Manipal Centre for Virus Research, Manipal University, Manipal, Karnataka, India-576104

In the recent years, the incidence of chikungunya cases has increased in India and other tropical countries with high attack rate. However, the evidence of other alphaviruses in India is unknown. So, the rationale of the study is to detect circulating alphaviruses in India. A retrospective cross-sectional study was conducted for the molecular detection of alphaviruses in acute febrile illness cases in India during the year 2015. A total of 902 serum samples archived at Manipal Center for Virus Research from Acute Febrile Illness (AFI) cases during the period, January 2015 to December 2015 were selected. Among the 902 AFI cases, 453 cases $(48.6 \%)$ were from Thirthahalli, 174 cases $(18.7 \%)$ from Mananthawady, 121 cases (13\%) from Sultan Bathery, followed by Hosanagara 82 cases $(8.8 \%)$, Soraba 22 cases $(2.4 \%)$, Sagara 33 cases $(3.5 \%)$, Valpoi 12 cases $(1.3 \%)$ and Mapusa 5 cases $(0.5 \%)$. Serum samples were extracted and the nucleic acid was screened for alphavirus using conventional reverse transcriptase polymerase chain reaction (RT-PCR). Further chikungunya real-time reverse transcriptase PCR was performed for all the alphavirus PCR positive cases. Out of 902 samples tested, 3 cases were tested positive for alphaviruses and the prevalence of alphaviruses among AFI cases were $0.32 \%$. Confirmation of all the positive cases was done by sequencing. This study documents that chikungunya virus is the circulating alphavirus among the selected AFI study population during 2015. It can provide valuable information to policy-makers for taking future decisions on alphaviruses in India.

An encounter with fractures among HIV infected individuals: a series of 13 cases from Coastal Karnataka, India

\section{Jutang Babat Ain Tiewsoh ${ }^{1}$, Beena Antony ${ }^{2}$, Rekha Boloor ${ }^{3}$}

Email ID for Correspondence: jbatiewsoh@ rediffmail.com

${ }^{1}$ Post Graduate Resident, Department of Medical Microbiology, Father Muller Medical College, Mangalore; ${ }^{2}$ Professor, Department of Medical Microbiology, Father Muller Medical College, Mangalore; ${ }^{3}$ Professor and Head of department, Department of Medical Microbiology, Father Muller Medical College, Mangalore

Human immunodeficiency virus (HIV) is no longer what it was 2-3 decades ago with the availability of anti-retroviral therapy. Mortality and morbidity have improved, but non-communicable diseases have become a common norm. Here, we describe 13 cases of fractures in HIV infected individuals. This retrospective study was carried out on HIV infected patients hospitalized for fractures in Father Muller Medical College Hospital, Mangalore from August 2013 to July 2015. 
13 cases were identified with a Male: Female ratio of 10:3, with 40-50 years age group the most commonly affected. 4 were known cases of HIV out of which 2 were on ART. The most common cause of fracture was road traffic accident $(n=7)$ followed by accidental fall $(n=6)$, long bones were more commonly affected with femur being the commonest. Treatment was done either by surgery or conservatively. 2 developed complication of osteomyelitis where Staphylococcus aureus was isolated while HBV infection was prevalent in 2 cases (15.38\%). We conclude that fractures in HIV are more common in older age group predominantly males where long bones are most commonly fractured. Hence, clinicians should keep in mind that fractures do occur in HIV infected individuals.

\section{Seroprevalence of HBV infection in HIV/TB co-infected individuals attending a tertiary care teaching hospital in South India}

\section{Jutang Babat Ain Tiewsoh ${ }^{1}$, Beena Antony ${ }^{2}$, Rekha Boloor ${ }^{3}$ \\ Email ID for Correspondence: jbatiewsoh@rediffmail.com ${ }^{1}$ Post Graduate Resident, Department of Medical Microbiology, Father Muller Medical College, Mangalore; ${ }^{2}$ Professor, Department of Medical Microbiology, Father Muller Medical College, Mangalore; ${ }^{3}$ Professor and Head of department, Department of Medical Microbiology, Father Muller Medical College, Mangalore}

According to the World Health Organization estimates, the human immunodeficiency virus (HIV) pandemic after nearly four decades of existence affected 36.7 million people by 2015 end. Tuberculosis being one of the commonest opportunistic infection resulting in many HIV deaths but hepatitis B virus (HBV) infection can also occur in these individuals as the general modes of transmission are similar to HIV. To investigate the seroprevalence of HBV in HIV-TB co-infected individuals. This descriptive cross-sectional study included 58 confirmed HIV-TB co-infected individuals. The serum samples from these patients were screened and confirmed for HBV following standard operative procedures. HIV-TB co-infection is most common in 41-50 years age group, with a male to female ratio 3.46:1. The seroprevalence of HBV in HIV-TB co-infected individuals is found to be $5.17 \%(\mathrm{n}=3)$ with all the three cases being males, married and above 35 years of age. The most common chief complaints in these patients is fever and loss of appetite. Extra-pulmonary TB was more common than pulmonary TB in a ratio of $2: 1$. We conclude that the seroprevalence of HBV in HIV-TB co-infected individuals was high. Hence, in a country like India where these three diseases are rampant, creating awareness and understanding about them is the need of the hour.

Clinical profile and genetic characterization of dengue viruses circulates in Chennai, India during 2011-2017

\section{Jeevan Malaiyan', Kamalraj Mohan', Sowmya Nasimuddin", Ananthan Balakrishnan ${ }^{2}$}

Email ID for Correspondence: kamalraj2019@gmail.com

${ }^{1}$ Department of Microbiology, Sri Muthukumaran Medical College Hospital and Research Institute, Chikkarayapuram, Chennai-600 069, India; ${ }^{2}$ Department of Human Genetics, Dr. ALM PG Institute of Basic Medical Sciences, University of Madras, Taramani, Chennai600 113, India

Dengue, which is one of the most prevalent mosquito borne infectious diseases, has shown rapid increase in Tamilnadu, India over the last 5 years. Worldwide, 390 million new cases are recorded annually. In
India, Tamilnadu and Kerala account for $70 \%$ of the cases with as many as seven out of every 10 dengue cases reported in the country are from these two states. The present study was done to investigate the trend of clinical profile and genetic characterization of dengue viruses (DENV) circulating in Chennai, India. Serum samples were collected from patients with clinically suspected cases of dengue fever over a period of 5 years (2011-2017). These samples were screened for hematological parameters, presence of dengue $\mathrm{NS}_{1}$ antigen, IgM and IgG antibodies, and amplification of specific genes by reverse transcriptase polymerase chain reaction (RT-PCR), followed by sequencing and phylogenetic tree construction. A total of 2299 samples were collected, of which 438 (19\%), were positive for acute Dengue fever. Most of common clinical features were fever with thrombocytopenia, which is observed in $90 \%$ of patients followed by myalgia, vomiting, headache, rash, abdominal pain, arthralgia and hemorrhagic manifestations with atypical manifestations were seen in the study. Among 438 positive cases, we did RTPCR only for 205 (47\%) samples (2015-2017), of which 186 (91\%) were positive for C-prM gene. Following sequencing and phylogeny, it was found that $35 \%$ of the samples belonged to serotype 1 whereas 23,4 , and $31 \%$ of serotypes belonged to serotypes 2,3 and 4 respectively. The consensus amino acid sequences of present study strains were compared with standard strains and it was found that few amino acid changes between strains. To conclude, atypical manifestations are increases and C-prM gene sequences revealed an active circulation of all serotypes in this region of India with few amino acid changes. Continuous epidemiological surveillance to monitor the incursion and spread of dengue virus genotypes in this region of India is needed.

Understanding the role of Staufen proteins in APOBEC3G stress granule-mediated regulation of $\mathrm{HIV}-1$ propagation

\section{Kannan Balakrishnan ${ }^{1,2}$, Ananda Ayyappan Jaguva Vasudevan²,} Carsten Münk ${ }^{2}$, Sharmistha Banerjee ${ }^{1}$

Email ID for Correspondence: bkannan7690@gmail.com ${ }^{1}$ Department of Biochemistry, School of Life Sciences, University of Hyderabad, India-500046; ${ }^{2}$ Heinrich-Heine-University Düsseldorf, Medical Faculty, Clinic for Gastroenterology, Hepatology and Infectiology, Düsseldorf, Germany

HIV-1 infectivity and propagation relies on host proteins. Staufen- 2 is one of such crucial host factors, we recently reported that Staufen-2 interacts with Rev of HIV and promotes RNA export activity of Rev. The Staufen proteins are main components of mRNA ribonucleoproteins (RNPs) complexes and play distinct roles in mRNA transport, translation and decay. Both Staufen-1 and Staufen-2 are recruited as RNA-protein complexes in stress granules in a mutually exclusive manner. APOBEC3G (A3G) is the best studied A3 DNA cytidine deaminase and the most potent restrictor factor of Vif deficient HIV-1. A3G encapsidated into HIV-1 particles exerts the antiviral function in the target cells upon infection by destabilizing viral genome. A3G exists in two major forms in cells, enzymatically active low molecular mass (LMM) form and catalytically inactive high molecular mass (HMM) form. A3G has also been reported to be associated with $\mathrm{P}$ bodies which contain mRNA processing proteins (polysomes). Earlier reports have shown that Staufen-1 containing RNA stress granules are a part of high molecular mass (HMM) complexes of cytoplasmic nucleoproteins A3G. Here, we have explored the interaction between A3G-Staufen-1 and Staufen- 2 in the cells by using co-immunoprecipitations. The interaction between Staufen-2 and A3G is found to be RNA-independent. In addition, like A3G, we found that Staufen-1 and Staufen-2 can incorporate into the viral particles. Further, we have made CRISPR/Cas9 based knockout 
cell lines to study the physiological role of Staufen protein in HIV-1 propagation. We have isolated RNP complexes of Staufen and A3G and analyzed for their interaction in HMM complexes. Ongoing experiments target the biological relevance of Staufen interaction with $\mathrm{A} 3 \mathrm{G}$ and HIV particles.

Measles virus infection among acute febrile illness cases with rash in wayanad district, Kerala during January 2015-July 2017

Karunakaran Kavitha, C Shilpa, Rao Chaitra, Jayaram Anup, Abdulmajeed Jazeel, S Aswathyraj, Devadiga Santhosha, Govindakaranavar Arunkumar

Email ID for Correspondence: kavisouth33@gmail.com Manipal Centre for Virus Research, Manipal University, Manipal, Karnataka, India-576104

Measles (rubella) is a highly contagious disease and is one of the leading cause of death in young children. Measles infection is usually associated with high grade fever, cough, coryza, conjunctival congestion and rash (Koplik Spots). Infection with measles virus can also lead to lifelong disabilities including brain damage, blindness and deafness in pediatric age groups. As per WHO, 8 clades designated A-H is recognized with 23 genotypes. In 2014, Manipal Centre for Virus Research initiated a hospital based laboratory supported acute febrile illness (AFI) surveillance that coordinated hospitals at the district and sub-district level in different states of India. A total number of 3689 AFI cases were enrolled in the surveillance from the 2 sentinel hospitals in Wayanad district, Kerala. Among these AFI cases, 121 cases had presented with rash, out of which 48 (39.6\%) cases presented with maculopapular rash were tested positive for measles virus. The median age of the confirmed cases was 17 years (IQR 9-23). The confirmed cases had clinical symptoms such as cough $(97.9 \%)$, coryza $(60.4 \%)$, and sore throat $(58.3 \%)$, breathlessness (4.2\%), conjunctival congestion (54.2\%), diarrhea $(29.2 \%)$, vomiting $(72.9 \%)$ and myalgia $(45.8 \%)$. Measles virus showed its peak activity during January to May. The study provides an outline on the prevalence of measles virus in Wayanad district of Kerala during January 2015 to July 2017. The findings of this study can attribute to understanding the epidemiology and the vaccine coverage which can contribute to the nation's goal of measles elimination.

\section{Involvement of RTK in the enhanced uptake of human adenovirus serotype 5 into THP1 cells}

\section{Lathika Valliyott, Ranjeet Dungdung, Rajendra Pilankatta}

Email ID for Correspondence: lathikav@cukerala.ac.in Department of Biochemistry and Molecular Biology, School of Biological Sciences, Central University of Kerala, Kasargod, Kerala, India-671314

Human adenovirus serotype 5 (AdV5) is non enveloped double stranded DNA viruses which are explored for vaccination as well as gene therapeutics. A major barrier that significantly impede the effectiveness of AdV5 mediated gene transfer is the seroprevalence of pre-existing anti-adenovirus neutralizing antibodies. Adenovirus serotype 5 infects a variety of human cells and tissues except the cells, which doesn't carry Coxsackievirus and Adenovirus Receptor (CAR). Meantime, it was reported that pre-existing anti-AdV5 antibodies enhance the uptake of AdV5 in CAR negative human monocytic cell lines such as U937 and THP1. In the current study, the involvement of host cell kinases in the antibody dependent uptake of AdV5 into CAR negative/Fc gamma RI and RII positive monocytes (THP1 cells) was investigated. A replication defective recombinant adenovirus 5 expressing GFP (AdV5-GFP) based screening system was used to map anti-AdV5 antibody in human sera using HeLa cells. All the tested normal healthy adult human sera showed the presence of varying titers of anti-AdV5 antibody. Also, there was an enhancement in uptake of AdV5-GFP complexed with the Ad5 immune sera into THP1 cells, possibly mediated through $\mathrm{F}_{\mathrm{C}}$ gamma RI and RII. Small molecule inhibitor based screening was done using src and tyrosine kinases (RTK) inhibitors against the uptake of AdV5 GFP into THP1 cells. Interestingly, a significant increase in the uptake of AdV5 GFP immune complex was observed in THP1 cells which were pre-incubated with the kinase inhibitor. These observations have implications in the development of Ad5 vector based therapeutics.

Seroprevalence of dengue and chikungunya co-infection in a tertiary care hospital, Mysuru

Manasa. P. Kumari, Dr. Amrutha Kumari B, Dr. Neetha Murthy. S, Shivaranjini. D. S, Dr. Anuradha K

Email ID for Correspondence: manasavinay22@gmail.com Viral Research and Diagnostic Laboratory, Department of Microbiology, MMC and RI Mysuru 570001

Arboviral infections like dengue and chikungunya are endemic in tropical and sub-tropical regions. Both infections share typical clinical manifestations and mode of transmission. Since the transmission is by common mosquito vector (Ae. aegypti) co-circulation of both viruses are prevalent in many geographical areas. In India, the history of coinfection dates back to 1964 . The present study was aimed to determine the seroprevalence of dengue and chikungunya co-infections. Hundred and eighty one patients presenting with high grade fever, headache, arthralgia, myalgia, rashes, etc. were included in the study. Serum samples were collected from June 2017 to September 2017 and tested for dengue and chikungunya by IgM ELISA. Among 181 samples tested; 61 (33.7\%) were positive for dengue, 36 (19.8\%) were positive for chikungunya and $19(10.4 \%)$ were positive for both. As the clinical features are same in both the cases, majority of CHIK infections may go undiagnosed in dengue endemic areas and vice versa in CHIK endemic areas. Therefore, it is advisable to test for both viruses if patients are presenting with high grade fever, headache, arthralgia, myalgia, rashes etc.

Vitamin D3 attenuates HSV-1 induced anterior uveitis: an experimental study

Mini P Singh ${ }^{1}$, Archit Kumar', Uma Nahar Saikia ${ }^{2}$, Tripti Rungta ${ }^{1}$, Shefali Saini ${ }^{1}$, Amod Gupta ${ }^{3}$, Reema Bansal ${ }^{3}$, R K Ratho 1

Email ID for Correspondence: minipsingh@gmail.com Departments of Virology ${ }^{1}$, Histopathology ${ }^{2}$ and Ophthalmology ${ }^{3}$ Post Graduate Institute of Medical Education and Research, Chandigarh 160012, India

The eye is an immune privileged site and protects itself from inflammation by regulating immunosuppressive factors. The activation of pattern recognition receptors (PRRs) by Herpes simplex virus (HSV) breaks this privilege and results in inflammation. These inflammatory responses can lead to blindness by damaging the ocular tissue. The immunomodulatory role of vitamin $\mathrm{D}$ has been seen in various diseases. In the present study, we investigated the role of 25-hydroxyvitamin D3 supplementation against HSV-1 induced anterior uveitis in rabbit animal model. The inoculation of HSV-1 into 
the anterior chamber of rabbit eye resulted in severe inflammation with significant upregulation of mRNA levels of various PRRs (TLR2, TLR3, TLR4, RIG-1, MDA5, STING) on day 1 post-infection (p.i.) and neutrophilic influx was observed. There was a significant production of type I interferon (IFNs) as well as pro-inflammatory cytokines. However, on day 5 p.i., upregulation of TLR4, NOD2, VDR and ILs-(6, 17A and 23A) was observed with lymphocytic predominance. The 25D3 supplementation significantly downregulated the mRNA levels of TLR2, RIG-1, MDA5, STING and proinflammatory cytokines. Further, the 25D3 supplementation downregulated the mRNA levels of ILs- $(6,17 \mathrm{~A}$ and $23 \mathrm{~A})$ on day 5 p.i. However, no change in the mRNA levels of TLR3, IFN $\alpha$, IRF7 was observed. Also, the 25D3 supplementation resulted in the decline in HSV-1 load in the aqueous humor. Hence, the present study postulates that 25D3 supplementation may have an immunomodulatory role in reducing the inflammatory responses produced after HSV-1 anterior chamber inoculation in experimentally induced HSV-1 anterior uveitis.

\section{Identification of $B$ and $T$ cell epitope on structural proteins of Crimean-Congo hemorrhagic fever virus}

\section{Neha Shrivastava, P. K. Dash}

Email ID for Correspondence: nehashrivastava.bt@gmail.com Division of Virology, Defence Research and Development Establishment, Jhansi Road, Gwalior-474002, India

Crimean-Congo hemorrhagic fever (CCHF) orthonairovirus is a tick borne viral disease of humans belonging to the family Nairoviridae, genus Orthonairovirus. It causes sporadic cases of severe illness across a huge geographic area. Despite the high mortality associated with CCHF, the biology and pathogenesis of the disease remain poorly understood. Thus, early diagnosis and vaccine development are critical for both patient survival and for the prevention of potential nosocomial infection and transmission. One of the indispensible and significant steps of vaccine development is the identification of possible epitopes, which assist the production of effective subunit vaccine. The recognition of exceedingly competent $B$ cell linear (continuous) or conformational (discontinuous) and cytotoxic T-lymphocytes (CTL) epitopes is the critical step in vaccine development. The present study was initiated to map the immunodominant epitopes present in the nucleoprotein and glycoprotein regions of the CCHF virus using different in silico approaches such a Immune Epitope Database (IEDB) and Ellipro. In the present study, a number of potential epitopes were identified which could serve as potential vaccine candidates. In addition, such an approach could also help in customizing novel peptide vaccines according to population genetics.

\section{DENV-2 infection and DENV-2 rNS1 treatment induces} alterations in vWF in endothelial cells in vitro

\section{Nitali Tadkalkar, Shobha Gangodkar, Sharda Prasad, Atanu Basu}

Email ID for Correspondence: nitali04@gmail.com

Electron Microscopy and Histopathology group, National Institute of Virology, 20-A, Dr. Ambedkar Road, Pune - 411001, India

Dengue is a major disease caused by the Dengue virus (DENV), with approximately 390 million new infections and 2 million severe (DHF/ DSS) cases every year. Dysfunction of vascular endothelial cells is a major event during DHF/DSS pathogenesis, but the mechanisms of these endothelial events are poorly understood. The DENV non- structural protein-1 (NS1), in its soluble form is known to be released during DENV infection and found in patient sera; its levels often correlating with disease severity. However, the mechanism by which NS1 contributes to disease severity is not known. In this study, we used correlative microscopy and biochemical techniques to characterize the alterations caused in key molecules essential for maintaining homeostasis of endothelial physiology; by DENV-2 infection and DENV-2 NS1 exogenous treatment, in primary human umbilical vein endothelial cells (HUVECs). We found evidence that the expression of $\mathrm{vWF}$, an important endothelial marker, is significantly altered in DENV infected and DENV NS1 treated endothelial cells; thus giving clues to underlying signaling cascades which may be significant towards the development for endothelial dysfunction in DHF/DSS.

\section{Human coronaviruses infection among acute respiratory illness} cases in India

Prabhu G Suresha, Devadiga Santhosha, Maity Hindol, Ramachandran Sanjay, Rao Chaitra, Govindakarnavar Arunkumar

Email ID for Correspondence: suresh.prabhu@manipal.edu Manipal Centre for Virus Research, Manipal University, Manipal, Karnataka, India-576104

Acute respiratory illness (ARI) is are major health problem in people of all age groups according to world health organization. Majority of the ARI is caused by viruses mainly Influenza, Rhinoviruses, Respiratory syncytial virus etc. Human coronaviruses also identified as group of viruses which has ability to infect humans and animals. Six human coronaviruses have been identified till date, which are $\mathrm{HCoV}$ 229E, HCoV-HKU1, HCoV-NL63, HCoV-OC43, Severe Acute Respiratory Syndrome coronavirus (SARS-CoV) and Middle East Respiratory Syndrome coronavirus (MERS-CoV). In 2014 Manipal Centre for Virus Research initiated the hospital-based laboratorysupported acute febrile illness (AFI) surveillance at District and subdistrict level sentinel hospitals across several states to map the aetiology of AFI cases. A total number of 27,586 acute febrile illness cases were enrolled in the surveillance during June 2014 to July 2017, of which 18,415 cases had acute respiratory illness. Out of these ARI cases, $144(0.8 \%)$ were positive for human coronaviruses. The median age of the confirmed cases was 25 (IQR 16-40). Among the confirmed cases 64 were positive for $\mathrm{HCoV}-\mathrm{OC} 43,21$ positive for HCoV-NL63, 27 were HCoV-229E and 32 cases were positive for HCoV-HKU1. The confirmed cases had clinical symptoms such as cough $(68.8 \%)$, coryza $(60.4 \%)$, sorethroat $(34.7 \%)$, breathlessness $(16.7 \%)$, myalgia $(77.8 \%)$, nausea $(39.6 \%)$, vomiting $(32.6 \%)$. Coronaviruses were seen throughout the year with peak activity during January to March. Though the prevalence observed in this study was less, it gave an insight into the role of human coronaviruses in acute respiratory illness. The findings from this study will form a baseline data for the future research on disease burden and epidemiology of human coronaviruses in India.

Human papillomavirus down-regulates transcription factor Nrf2 and depletes GSH levels: a possible mechanism for enhanced sensitivity to therapy in HPV-positive cancers

\section{Pushkal S R, Devanand Devegowda, Rajesh Thimmulappa}

Email ID for Correspondence: pushkal.sr@jssuni.edu.in 
Center for Excellence in Molecular Biology and Regenerative Medicine, Department of Biochemistry, JSS Medical College, JSS University, Mysuru-15

Human papillomavirus (HPV) is a known cause of $98 \%$ of cervical cancers, $50 \%$ of Head and Neck cancers and $25-60 \%$ of other anogenital cancers. Elevated oxidative stress is a notable characteristic feature of HPV infected cancer cells partly due to activation of NADPH oxidase and glutathione (GSH) depletion. To counteract the oxidative stress, cell upregulates transcription factor NRF2, which transcribes nearly all the cellular antioxidants including GSH biosynthesis and phase II detoxification enzymes. Elevated Nrf2 signalling is a mediator of radio resistance and chemo resistance in human cancers whereas down-regulation of Nrf2 is shown to sensitize cancer cells to treatment. When compared to HPV - ve, HPV +ve cancer patients show better responses to radio- and chemotherapy, however the mechanisms are unclear. We hypothesize that HPV infection down-regulates Nrf2 signalling that depletes cellular antioxidants and sensitizes to radio- and chemotherapy. To test the hypothesis, we evaluated Nrf2 signaling in HPV + ve ( $\mathrm{SiHa}$ and $\mathrm{HeLa}$ ) and HPV - ve (C33A) cervical cancer cell lines by assessing i) expression of Nrf2, Keap1 and selected Nrf2 regulated antioxidants genes (NRF2, NQO1, GCLC and GCLM and HO-1) by quantitative PCR ii) Nqo1 enzyme activity, iii) GSH levels. QPCR data analysis revealed marked decrease in expression of NRF2, NQO1, GCLC and GCLM genes while elevated expression of KEAP1 (Nrf2 inhibitor) gene in HPV + ve cells as compared to HPV -ve cells. In agreement with $N Q O 1$ gene expression, NQO1 enzyme activity was markedly depleted in HPV + ve as compared to HPV - ve cells. Similarly, we observed marked reduction in levels of GSH in HPV + ve cell as compared to HPV - ve cells. In conclusion, this study suggests that HPV infection inhibits Nrf2 regulated antioxidant defences and enhance sensitivity to therapy in HPV associated cancers. Our ongoing studies are focused on delineating underlying mechanism how HPV modulates Nrf2 signaling.

\section{Dengue 2 virus NS4A protein interacts with GIV}

\section{Ranjeet Dungdung, M Nageswar Reddy, KV Sharath Rao, Aswathi KS, Lathika Valliyott, Rajendra Pilankatta}

Email ID for Correspondence: ranjeet777@cukerala.ac.in Department of Biochemistry and Molecular Biology, School of Biological Sciences, Central University of Kerala, Kasargod, Kerala, India-671314

Dengue is caused by mosquito-borne viral infection transmitted by Aedes aegypti, which poses a global health threat to humans, affecting almost 2.5 billion people due to its high adaptability to urban communities. Dengue virus (DV) contains a positive sense $11 \mathrm{~kb}$ RNA which is replicating in the membranous compartments of the cytoplasm of the infected cells. The genome is translated into a single polyprotein that is cleaved into three structural proteins (capsid [C], envelope $[\mathrm{E}]$, and membrane $[\mathrm{prM} / \mathrm{M}]$ ) and seven non-structural proteins (NS1, NS2a, NS2b, NS3, NS4a, NS4b, NS5). The nonstructural protein (NS) 4a is highly hydrophobic and associates with membranes via its internal hydrophobic regions. Recently, it was reported that NS4a plays an important role in the induction of autophagy in Dengue 2 virus infected cells. It is also important to note that $\mathrm{G} \alpha$-interacting, membrane vesicle-associated protein (GIV, a.k.a. Girdin), a non-receptor guanine nucleotide exchange factor for Gai3, plays a key role in regulating autophagy. In this context, the current study was carried out in BHK21 cells containing packaging deficient stably replicating DV2 genome (DV2-BHK-Replicon cells) and DV2 infected BHK21 cells, to understand the interaction between NS4a and Girdin protein. Western blot analysis showed the significant enhancement in the expression level of GIV in the DV2-BHKReplicon cells as compared normal BHK21 cells. The upregulation was hampered by the addition of Mycophenolic acid (MPA), indicating the involvement of GIV in the replication of DV2 genome. Immunostaining study showed enhanced level of perinuclear distribution of GIV in the DV2-BHK-Replicon cells and DV2 infected cells. Importantly, the immuno-precipitation experiment revealed the interaction of GIV with NS4a. Therefore, further studies are warranted to understand the regulation of DV2 induced autophagy mediated through GIV.

\section{Case report: detection of Zika virus infection in South India}

\section{Govindakarnavar Arunkumar, Jazeel Abdulmajeed,} Anup Jayaram, Santhosha Devadiga, Aswathyraj S, Robin S

Email ID for Correspondence: robin.s@manipal.edu Manipal Center for Virus Research, Manipal University, Manipal, Karnataka, India-576104

Zika virus (ZIKV) is an emerging pathogen. The presence of competent mosquito vectors (Aedes aegypti and A. albopictus) makes India vulnerable to human Zika infection. However, the magnitude of this virus circulation and geographic spread is notevident due to the lack of epidemiological records and serological evidence. In May 2017, MoHFW reported three cases of Zika from Bapunagar of Gujarat. Laboratory confirmation (RT-PCR) was done at B.J. Medical College, Ahmedabad and re-confirmed at National Institute of Virology (NIV), Pune. In this case report, we like to demonstrate the first laboratory-confirmed indigenous case of Zika in the state of Tamil Nadu. The emergence of Zika outside of its previously known geographic range should prompt awareness of the potential for Zika to transmit to new areas. Zika in Tamil Nadu was detectedthrough one of the 33 Acute Febrile Illness (AFI) sentinel surveillance sites established by Manipal Center for Virus Research (MCVR), Manipal University, in 10 Indian states. The patient was a 27 -year-old, male, presented with symptoms of fever, headache, photophobia, retro-orbital pain, red eye, myalgia and general weakness admitted to Anchetty PHC, Krishnagiri. The patient had no history of travel to Zika affected countries. On investigation, blood and urine samples were found positive for Zika virus by Trioplex real-time RT-PCR (CDC Atlanta USA). Sequencing using ABI 3500 XL genetic sequenceanalyzerconfirmed circulating virus belongingto the Asian lineage (Gene accession number: MCVR-MG287146/India/2017). Investigation suggests evidence of low-level transmission of Zika in Krishnagiri and the probability for the incidence of new cases. This Zika threat can be mitigated by strengthening the surveillance activities and strategic approaches for mosquitocontrol in the affected area.

\section{Investigating host response to Chandipura virus infection}

\section{Sachendra S. Bais, Yashika Ratra, Payel Roy, Soumen Basak}

Email ID for Correspondence: sachendrabais@nii.ac.in Systems Immunology Laboratory, National Institute of Immunology, New Delhi, India

Chandipura virus (CHPV) is an emerging human pathogen responsible for several of the recent encephalitis outbreaks in India. CHPV belongs to Rhabdoviridae family and vesiculovirus genera and possesses a single-strand negative sense RNA genome. An understanding of the cellular signaling pathways underlying the anti-viral immune 
response is important to comprehend fully viral-meditated pathogenesis. In general, endosomal TLR3 as well as cytosolic RIG-I sense invading RNA viruses and transmit signals for activating RelA/NF$\kappa \mathrm{B}$ and IRF3 transcription factors in the nucleus. These factors collaborate to induce the expression of a diverse set of anti-viral genes, including that encoding Interferon- $\beta$. However, dynamical control and amplitude of cellular responses often vary for different RNA viruses owing to virus-specific signaling mechanisms. Despite human health relevance, the specific molecular mechanism underlying hostresponses to CHPV infection remains elusive. More so, genetic dissections of the role of individual host components in the immune response to RNA viruses are wanting. Here, we utilized genetically tractable and biochemically amenable mouse embryonic fibroblast (MEFs) based system for analyzing host-responses to CHPV. Corroborating findings involving other RNA viruses, CHPV induced the RelA activity in MEFs via the canonical NF- $\kappa B$ pathway and also triggered the IRF3 activity in a TBK1/IKK $\varepsilon$ dependent manner. A lack of Interferon- $\beta$ production upon CHPV infection of Rela $^{-1-}$ cells substantiated a necessary role of the NF- $\kappa \mathrm{B}$ pathway in antiviral hostresponse. Unexpectedly we also observed that infection of RelA-deficient MEFs led to a decreased yield of progeny CHPV particles despite abrogated IFN- $\beta$ expressions. Our mechanistic study indicated that this pro-viral NF- $\kappa \mathrm{B}$ function was linked with the ability of the RelA factors in suppressing cellular death. In sum, multifunctional transcription factors, known for their involvement in the anti-viral host responses, are also exploited by RNA viruses for their own benefit.

\section{Detection and identification of prevalent Rotavirus and Norovirus strain from raw street food in Kolkata}

\section{Sayantan Adhikary, Ahana Bhattacharya, Bagisha Maitra Sulagna Mandal, Trishita Banerjee, Aparna Mukhopadhyay}

Email ID for Correspondence: sayantan.rs@presiuniv.ac.in Department of Life Sciences, Presidency University, Kolkata, West Bengal, India-700073

Although, the general awareness about bacterial gastroenteritis is well established, people lack information about virus mediated gastroenteritis and its consequences. Rotavirus and Norovirus infection remains a significant clinical problem throughout the world, infecting children and adults, despite socio-economic status or environmental conditions. These viruses are transmitted mainly via the feco-oral route and sources include raw, minimally processed food such as fruits and vegetables, salads, shellfish or contaminated water. An attempt was made to isolate the common food-borne Rotavirus and Norovirus from vegetables generally eaten raw like coriander leaves, salads, cut-fruits, dates, grapes from susceptible areas mainly from busy street-sides and unhygienic, highly polluted, crowded and commonly visited market places in Kolkata where they are sold in an exposed state. Virus isolation was performed by surface washing of collected raw fruit/vegetable samples with Tris-glycine, then precipitating virus particles (if present) with Polyethylene Glycol (PEG) followed by purification, concentration using $0.2 \mu \mathrm{m}$ filter and viral RNA isolation using Trizol. Genetic analysis of the RNA genome was carried out by denaturing UREA Polyacrylamide Gel Electrophoresis (PAGE) to characterize the electrophoretic patterns. Strain specific primers were designed and genotyping was carried out by RT-PCR techniques of specific sequences of the viral genome. Preliminary results showed the presence of G9 Rotavirus and Genotype II Norovirus strains. From these results we can conclude that common roadside raw food particles are often infected with Rotavirus and Norovirus and such food particles must be avoided for leading a healthy life. Further studies are being carried on, which includes sequencing of the viral genome, and cloning techniques for authentication and confirmation of the obtained results.

Hepatitis A outbreak traced back to ice used in fruit juice in Nellikuzhi panchayat, Ernakulam district, Kerala state

Sheik Shahin ${ }^{1}$, HossainSheik Shah ${ }^{2}$, AbdulmajeedJazeel ${ }^{1}$, JayaramAnup ${ }^{1}$, Robin $S^{1}$, Chandra BharaniKiran ${ }^{1}$, DevadigaSathosha $^{1}$, NitturSudheesh ${ }^{1}$, Govindakarnavar Arunkumar ${ }^{1}$

Email ID for Correspondence: shahin.sheik@manipal.edu ${ }^{1}$ Manipal Centre for Virus Research, Manipal University, Manipal, Karnataka, India -576104; ${ }^{2}$ Prasanna School of Public Health, Manipal University, Manipal, Karnataka, India - 576104

Viral hepatitis affects 400 million people globally and the annual burden of Hepatitis A is estimated to be 1.4 million cases. In the year 2015, Integrated Disease Surveillance Program (IDSP) reported 86 outbreaks of viral hepatitis (Excluding Hepatitis-C) of which 20 (23\%) outbreaks occurred in Kerala. HAV has a clinical spectrum ranging from mild disease to severe illness that lasts up to 2 weeks. In September 2016, a clustering of HAV was reported from Nellikuzhi Panchayat, Ernakulam districtof Kerala to the district health office by a private hospital that lead to this outbreak investigation. Manipal Centre for Virus Research investigated the outbreak during 16th-23rd December 2016. A total of 289 suspected cases were reported with an attack rate of 1.20 per thousand populations. The highest number of suspected cases was found in Company pady, 35 cases (12\%), Nellikuzhi 33 cases $(11.5 \%)$ and Edanadu, 22 cases $(8 \%)$. The first case reported has an onset history of 14 November 2016. The last case was reported with onset history of 14 December 2016. Blood and Stool specimens, environmental samples (Well water) were obtained from the suspected cases and tested for HAV Real Time PCR. Confirmed Hepatitis-A cases $(\mathrm{N}=41)$ reported fever $(88 \%)$, vomiting $(51 \%)$ and jaundice $(66 \%)$. All the confirmed cases had a history of consuming at least a single serving of any food, water and beverage in a newly opened restaurant (Odds ratio: 14.23, CI 4.09, 49.46). We hypothesized the possible exposure of the outbreak is due to any of the food, water or any beverage served at the restaurant. To prove the hypothesis, we conducted a case control study and matched human specimens with environmental samples. Multivariable regression analysis identified consumption of fresh juice (OR 22.5, CI 6.34, $79.65)$ as the major exposure. The Hepatitis A viruses recovered from cases belonged to genotype IIIA and were phylogenetically $100 \%$ similar to the Hepatitis A virus recovered from ice collected from the freezer of the juice station of the restaurant; and this ice was prepared from the well water. The investigation concluded that the newly opened restaurant serving water and beverages with ice prepared from water from the nearby well as the source of HAV outbreak.

\section{Zika: an emergent threat to human health}

\section{Shambhavi Rao}

Email ID for Correspondence: shambhavi2536@gmail.com Amity University, Uttar Pradesh

Zika was first discovered in 1947, belong to family Flaviviridae; genus Flavivirus in Zika forest of Uganda, so it's not an old but a reemergent virus. The first human infection reported dates back in 1952, in Nigeria. Since then the virus has caused many sporadic infections but abominably came into limelight when it caused three major outbreaks: in Yap islands 2007, in French Polynesia 2013 and the most 
recent major outbreak in the Americas 2015; after which it was declared as a Global Health Emergency, by WHO. The strains isolated from American as well as Pacific Island were relatively closer to Asian lineage strains. The prevalent Asian strain caused high mortality and morbidity rates since then around the world, especially the incidences of microencephaly in the new borns caught major health and governmental concerns. As a result the research engrossing new techniques, diagnostics and therapeutics targeting the re-emergent infection became the need of hour. It's important to study and develop combating measures against Zika in India as, firstly country shares similar geographic and climatic conditions as of those where Zika Virus is prevalent, secondly the main vector carrier Aedes spp. of the virus is in abundance in India and lastly return of athletes from Rio, Brazil 2016 that could possibly be the source of infection in the country. Till today, in India its presence has not been reported, but, several points need to be addressed on a priority as country preparedness. To date there are no commercially available kits (Approved or validated) for serological determination of Zika. Also, the flavivirus cross reactivity is well documented and hence there is an urgent need for development of specific and sensitive sero-diagnostic reagents and study of epidemiology to identify recent infection in patients and also to design proper management of disease.

\section{Human adenovirus infection among acute respiratory illness cases} in India during June 2014-July 2017

\section{Shilpa C, Karunakaran Kavitha, Rao Chaitra, Jayaram Anup, Sheik Shahin Auti Milind Amogh, Govindakarnavar Arunkumar}

Email ID for Correspondence: shilpapast@gmail.com

Manipal Centre for Virus Research, Manipal University, Manipal, Karnataka, India-576104

Human adenoviruses can cause wide range of diseases including respiratory illness, such as common cold, croup, bronchitis or pneumonia, gastroenteritis and conjunctivitis (pink eye). The adenoviruses includes a large group of agents that are isolated from both human and animals. So far, a total of 68 types of HAdVs have been identified and has been classified into seven species or subgroups A-G. Manipal Centre for Virus Research initiated a hospital- based laboratory supported acute febrile illness (AFI) surveillance study in 2014, at district and sub-district level sentinel hospitals in different states of India, to monitor and map the aetiologies of AFI cases. A total number of 27,586 acute febrile illness cases were enrolled in the surveillance during June 2014 to July 2017, out of which 18,415 cases had acute respiratory illness. Out of these ARI cases, $154(0.84 \%)$ were positive for human adenovirus. The infection was primarily confined to paediatric age group. The median age of the confirmed cases was 10 years (IQR 5-18). Out of the 154 positive cases, 87 (57\%) were male and 67 (44\%) female. Among the confirmed cases, 41 cases $(27 \%)$ were from Karnataka. The confirmed cases had clinical symptoms such as cough $(53 \%)$, coryza $(42.8 \%)$, sore throat $(41 \%)$, breathlessness $(6.5 \%)$, myalgia $(67.5 \%)$, vomiting $(40.2 \%)$, conjunctivitis (12.9\%), and diarrhoea (11\%). Adenoviruses were circulating in the community throughout the year, with its peak activity during January to May. A low prevalence of HAdVs were observed in the study, which gave an overview about the infection caused by HAdVs. This study can help in further research on the epidemiology and disease burden associated with adenoviruses especially in the paediatric age in India.

\section{Persistence of specific IgM antibodies in chikungunya}

\section{Shweta Chelluboina, Robin S, Aswathyraj, G. Arunkumar}

Email ID for Correspondence: shwetachelluboina@gmail.com Manipal Centre for Virus Research, Manipal University, Manipal576104

Chikungunya virus (CHIKV) disease is a mosquito-borne viral illness caused by an arthritogenic alphavirus. It is often associated with chronic arthritic symptoms that persist for months. Since its reemergence, Chikungunya has become one of the major public health problems in India. During the year 2010, around 48,176 cases were reported in India. Since then, there was a slight decline in the number of reported cases. However, this number has increased drastically since 2015. In general, IgM is the first antibody to appear within a week post any infection and declines within 2-3 months. Contrary to this common belief, there are reports in the literature documenting the presence of IgM against chikungunya virus for a longer duration. There is no such data on the same in Indian population. We have conducted a study to document the presence of specific IgM antibody among Chikungunya cases. These cases were enrolled on confirmed lab diagnosis by Real-Time PCR. Serum samples were collected from the time of diagnosis, at $1,8,10$ and 12 months post infection. The samples were tested using IgM capture ELISA technique (NIV Pune). We were able to document the presence of anti-chikungunya IgM in a majority of patients $(76 \%)$ for up to 10 months post infection. Further research is required to understand the reason behind such persistence. Hence, detection of Chikungunya IgM in acute febrile illness for Chikungunya infection in endemic areas should be interpreted cautiously as it may lead to misdiagnosis of cases. Real-Time PCR is more reliable for detection of acute Chikungunya cases in endemic areas while IgM detection may be useful in identifying exposure to the disease.

Co-infection of dengue and chikungunya: a study from a tertiary care centre in South India

\section{Shantala GB, Sathyanarayan MS, Shwetha JV and Ambica R}

Email ID for Correspondence: shwethanadig2000@gmail.com Bangalore medical college and research institute, Bangalore

Dengue and Chikungunya shares common transmitting vectors and shows similar clinical signs during the early stages of infection. Coinfection of Dengue and Chikungunya may occur in susceptible population where the diseases are endemic. To detect co-infection of dengue and chikungunya in patients presenting with acute febrile illness to a tertiary care centre. In the present study, 56 patients presenting with acute febrile illness were included. The serum samples were collected after obtaining the informed consent. They were tested for the detection of chikungunya infection by serum IgM ELISA and tested for the presence of antibodies against dengue by IgM ELISA. The serum samples are under process for detection of co infection by molecular methods. Out of 56 samples, 28 (50\%) showed IgM antibodies against chikungunya and $17(30.35 \%)$ were positive for dengue IgM antibodies, of which 10 (17.86\%) samples showed positive for both chikungunya and dengue IgM antibodies. The serum samples are under process for detection of co-infection by molecular methods. Co-infection of dengue and chikungunya were detected in nearly $1 / 3$ of patients presenting with acute febrile illness. Heightened awareness of co-infection among the clinicians is required to investigate for both simultaneously which has an impact on patient management. 
Development of a real-time RT-PCR assay that distinguish between alkhurma hemorrhagic fever virus and kyasanur forest disease virus

Sudheesh. $\mathbf{N}^{1}$, Babak Afrough ${ }^{2}$, Kewin Bewley ${ }^{2}$, Roger Hewson ${ }^{2}$, G. Arunkumar ${ }^{1}$

Email ID for Correspondence: sudheesh.n@manipal.edu ${ }^{1}$ Manipal Centre for Virus Research, Manipal University, Manipal, Karnataka, India; ${ }^{2}$ Microbiology Services, Public Health England, Porton Down, Wiltshire, United Kingdom

Kyasanur forest disease virus (KFDV) and alkhurma hemorrhagic fever virus (AHFV) are closely related tick-borne viruses of the genus Flavivirus. Both cause significant morbidity and mortality with a mortality rate of $2-10 \%$. KFDV and AHFV share high sequence homology ( $>92 \%$ nucleotide similarity) but maintained distinct geographical locations India and Saudi Arabia. Human cases of KFD have been reported in over five states across the Western Ghats region of India and AHF in across Saudi Arabia. The principal tool for the diagnosis of these diseases is by Real Time RT- PCR assay, which is sensitive and relatively specific but fails to distinguish these viruses. Since they are genetically very similar, there could be a possibility that these viruses were prevalent in other areas for years but was never detected due to the absence of a proper diagnostic tool. Therefore, the objective of the study is to develop a rapid and sensitive $\mathrm{qPCR}$ assay that differentiate between these two viruses. Multiple sequence alignment of KFDV and AHFV was done, and melt curve analysis of NS5 gene was performed using the uMelt software. Based on this findings, primers and probes were synthesised and analysed by PCR using AHFV and KFDV standard strains obtained from Public Health England, UK. The assay is capable of differentiating between KFDV and AHFV. The limit of detection of the assay is six copies of target RNA, and the assay shows no crossreactivity with other tick-borne viruses or viruses causing similar human disease. To best of our knowledge, this is the first probe based real-time PCR assay capable of differentiating AHFV and KFDV infection, and offers a simple, cost- effective diagnostic assay for the detection of these viruses.

\section{Genotypic characterization of dengue virus-2 from Meghalaya, India}

\section{Sumi Chetry, Siraj Ahmed Khan, Prafulla Dutta}

Email ID for Correspondence: sumichetry@gmail.com Arbovirology group, Entomology Division, Regional Medical Research Centre, North East Region (ICMR), Dibrugarh- 786001

The Northeastern region of India has been experiencing dengue almost every year since a decade. In 2016, alongside Chikungunya, a Dengue outbreak also occurred in Tura, Meghalaya. Information about Dengue virus (DENV) and its genetic make-up is not known from this region. Thus, we attempted to identify the serotype and genotype of DENV circulated during the outbreak. A total of 62 Dengue/Chikungunya suspected cases reporting at the Tura Civil Hospital, Meghalaya was investigated for Dengue specific IgM and/or NS1 ELISA. Further, all the serum samples positive for NS1 ELISA were processed for serotyping analysis and C-prM gene junction amplification by RT-PCR using specific primers. The amplified products were sequenced and the obtained sequences were used for phylogenetic analysis to determine the genotype of the virus. A phylogenetic tree was constructed by Maximum Likelihood method based on General Time Reversible model using gamma distribution with an invariant site in MEGA version 6.0. Out of the 62 samples processed, 13 were positive for Dengue (IgM ELISA: 5; NS1 ELISA: 8). Among the NS1 positive samples processed for serotyping, 7 samples showed amplification and all were positive for DENV-2. Genotypic characterization of the C-prM gene junction revealed circulation of Genotype-IV of DENV-2 during the outbreak. Knowledge of the circulating genotypes within serotypes of DENV is helpful for planning an effective clinical management of the infection where more than one serotype and genotype exists. This is the first serotyping and genotyping report of DENV from the Meghalaya state of India. Further research to characterize the different DENV serotypes and genotypes prevalent in the other Northeastern states are within the scope of this study.

\section{In-vitro evaluation of essential oils and their blend formulation} against influenza A H1N1

\section{Sweta T. Kothari, Pavankumar Todkar, Surabhi Rode, Rashmi Yadav, Durga Bethala}

Email ID for Correspondence: surabhirode@gmail.com Haffkine Institute for Training, Research and Testing, Parel, Mumbai400012

Influenza is one of the major causes of high global mortality and morbidity due to acute respiratory infections. Antigenic shifts and drifts in Influenza result in devastating pandemics and epidemics, due to the absence of pre-existing immunity against the new viral subtypes. Therefore, in conjunction with the available antiviral drugs there is a need to come up with preventive measures in order to circumvent the recurring influenza epidemics. Essential oils have distinctive antimicrobial, anti-mycotic and antiviral properties which have recently gained importance in the pharmaceutical field as a green alternative. As influenza mainly targets the upper respiratory tract of the host, the aim of the study was to prepare essential oils based emulsions and study their antiH1N1 properties. The preparation may be then used to prepare stable formulation as a gargle. Influenza A H1N1 virus (lab isolate) was propagated in Madin Darby Canine Kidney (MDCK) cells and viral titre was determined using RT-PCR method. Evaluation of cytotoxicity of clove oil, eucalyptus oil and cinnamon oil formulation and blend of these essential oils was carried out on MDCK cells using MTT assay and virucidal activity was determined using EN14476 standard protocol which was compared with the antiviral drug Oseltamavir. CC50 values of the formulation of Eucalyptus oil, Cinanamon oil, Clove oil and blend was found to be $0.09 \mathrm{mg} / \mathrm{ml}, 0.195 \mathrm{mg} / \mathrm{ml}, 0.163 \mathrm{mg} / \mathrm{ml}$ and $0.89 \mathrm{mg} / \mathrm{ml}$. respectively. Clove oil showed highest log reduction (3.625) followed by blend formulation of the three oils (2.375), eucalyptus oil (1.70) and cinnamon oil (0.875) being minimum. To focus on complete product based formulation, it is essential to further elucidate the active constituents of the essential oils with their mechanism of action.

A mathematical approach to the incidence of dengue: a skew hart analysis in dengue pattern recognition

\section{Tabasum Begum M, Sumana M.N.}

Email ID for Correspondence: tabasumbegum2013@gmail.com Department of Microbiology, JSS Medical College and Hospital, JSS University, Mysore

Dengue infection is an emerging disease and is a major public health problem in India. Globally the incidence of dengue has increased in recent years in terms of morbidity and mortality. The present study aimed to understand the suspected dengue pattern for the year 2015, 
2016 and 2017 in Mysore as Mysore being one of the dengue endemic districts of India. A total of 22,949 dengue suspected cases were collected from a tertiary care hospital, Mysore, South India. Based on the year of occurrence, the data were separated into 3 years and were analyzed using Skew hart technique, Poisson tests, Phase technique and c-chart. Suspected dengue in 2015 was in control with the upper limit at 164.45. In 2016, there was an upward shift with process mean 225.145 cases per inspection unit. In 2017, there was a highly significant upward shift in process mean and most of the points were above the revised control limits. Skew hart chart is currently one of the most popular statistical quality control charts in practice. The purpose of control chart is to estimate the process average and variation. It has an advantage of plotting data time to understand the process. The technique can be useful in monitoring dengue infection in future. In conclusion, Dengue is a rapidly emerging viral disease increasingly reported from many countries particularly from India. Dengue affected predominantly males and active adult population. A seasonal trend was observed for dengue infections with maximum cases in post monsoon and late monsoon months, which coincide with, increased breeding of mosquitoes during these seasons. Therefore, control of the vector population is the key to prevent the dengue outbreaks.

Trend of dengue and chikungunya virus co-infection in Odisha, India from 2013 to 2016

\section{Tanuja Saswat $^{1}$, Sagarika Muduli ${ }^{1}$, Nalini Sahoo ${ }^{2}$, Nagen Kumar Debata ${ }^{2}$, Soma Chattopadhyay ${ }^{1}$}

\section{Email ID for Correspondence: tanusaswat@gmail.com}

${ }^{1}$ Infectious Disease Biology, Institute of Life Sciences, Nalco Square, Bhubaneswar 751 023, Odisha, India; ${ }^{2}$ Department of Microbiology, IMS and Sum Hospital, Kalinga Nagar, Bhubaneswar 751003, Odisha, India

Dengue virus (DENV) infection is endemic in different parts of India and because of similar primary signs and symptoms Chikungunya virus (CHIKV) is mostly undiagnosed. Hence, we investigated 1318 Dengue suspected cases in a hospital based cross-sectional study in Odisha to evaluate single infection of DENV or co-infection of DENV and CHIKV from 2013 to 2016 by serology, RT-PCR and sequencing.

After screening, it was observed that $301(22.8 \%)$ samples were positive for DENV infection only, $62(4.7 \%)$ were positive for CHIKV infection only and interestingly, 41 (3.1\%) patients were coinfected with both DENV and CHIKV. After sequence analysis, it was noticed that DENV serotypes 1,2 and 3 were present and East Central South African type of CHIKV strain was found in studied population. Further, mutational analysis of E1 gene of CHIKV strains revealed that two consistent mutations (M269 V, D284E) were present in the circulated strains. Similarly, the comparative analysis of CprM gene of DENV serotype 2 strains showed the presence of four consistent mutations (M104 V, V112A, K166 N, and F169L). Additionally, it was noticed that male (age group between 16 and 30) were more infected with DENV and CHIKV than female and the disease occurrence was predominant mostly in the month of August and September. During this study, a significantly high number of DENV infected patients $(35.8 \%)$ were co-infected with CHIKV in 2013 but the co-infection was decreased from 7.5 to $3.4 \%$ and then $1.6 \%$ in 2014, 2015 and 2016 respectively. Thus, this study provides clear epidemiological picture of the above mentioned arbovirus which emphasizes the need of a routine diagnosis of CHIKV along with DENV febrile patients for early and proper recognition of infecting pathogen, correlate the clinical symptoms and proper patient care.
Molecular detection of human papillomavirus (HPV) in females attending OBG OPD and assessment of risk factors for HPV infection: a study from coastal Karnataka

\section{Varsha Saxena ${ }^{1}$, Abdulla Mehnaz ${ }^{2}$, Vidya Pai ${ }^{1}$, Rajagopal $\mathbf{K}^{2}$}

Email ID for Correspondence: varsha.saxena87@gmail.com ${ }^{1}$ Department of Microbiology, Yenepoya Medical College, Mangalore, Karnataka; ${ }^{2}$ Department of OBG, Yenepoya Medical College, Mangalore, Karnataka

Cervical cancer is the fourth common malignancies in world and Human papillomavirus (HPV) infection may lead to the development of the precancerous and cancerous lesions of the cervix. Therefore, the aim of the study was to evaluate the presence of HPV in women attending Gynecology OPD for any reason. A prospective study was conducted from November 2016 to September 2017. A complete clinical history and demographic status of 90 subjects was recorded of which total 50 tissue biopsies and 40 Cytobrush samples were collected. Results of histopathology and cytology (PAP smear) for biopsies and cytobrushes respectively were recorded from the Medical Record Department. Cytobrush samples were taken from the patients before Pap smear sampling during the routine examination. Further PCR was performed for the presence or absence of HPV in all samples. Early sexual debut, number of parity, hygiene, no regular screenings, use of cotton cloth vs. sanitary pads were the most important risk factors for the HPV infection in the present study. Out of 90 suspected cases, 50 were tissue biopsies, of which 15 had cervical carcinoma, 1 had CIN-II and 34 had chronic cervicitis. Whereas 40 were cytobrushes, of which 32 were negative for intraepithelial lesions/malignancy, 2 had ASCUS, 3 had inflammatory smear with reactive atypia and 3 had inflammatory smear. PCR results confirmed only $11 \mathrm{HPV}$ positives among 50 tissue biopsies and among 40 cytobrush samples only 6 were positive for HPV. In our locality the prevalence of HPV is low as compared to western countries and there is absolutely no awareness regarding HPV infection. Overall, these findings could have important implications for the prevention of cervical cancer.

RIG-1 inflammasome mediating HEV pathogenesis in acute viral hepatitis patients by interleukin-1 $\beta$ inflammatory cytokines

\section{Vikram Thakur', Radha Kanta Ratho', Mini P Singh", Y K Chawla ${ }^{2}$}

Email ID for Correspondence: vik5atif@gmail.com Departments of Virology ${ }^{1}$ and Hepatology ${ }^{2}$, Post Graduate Institute of Medical Education and Research, Chandigarh, 160012, India

Viral hepatitis $\mathrm{E}$ is known for its extensive epidemic potential in developing countries. One third of the global population is infected with Hepatitis E virus (HEV). Clinically the disease ranges from acute self-limiting hepatitis to acute liver failure (ALF) in pregnant women with $30 \%$ mortality to chronic infection in transplant patients, although the pathogenetic mechanisms of disease are not fully understood. Recently, the innate immune inflammasome signaling cascade shown to mediates the production of proinflammatory cytokines interleukin-1beta (IL-1 $\beta$ ) and IL-18 in other viral infection. The goal of this study was to determine the key inflammasomes mediating HEV infection. Blood samples from 85 clinically suspected viral hepatitis patients were screened for anti-HEV IgM, of which only 14 were positive for both anti-HEV IgM and HEV RNA and categorized as acute viral hepatitis (AVH). 11 individuals negative for all the viral markers with normal CRP were enrolled as healthy controls. Peripheral blood mononuclear cells (PBMCs) were isolated 
from $6 \mathrm{ml}$ blood using Ficoll-hypque density gradient centrifugation method. Approximately $2 \times 105$ PBMCs were processed for RNA extraction using TRIzol method and RNA integrity, quality and quantity were assessed by denatured gel and nano-drop respectively. Relative gene expression for NLRs (NLRP1, NLRP3 and NLRC4), RLRs (IFIH-1, DHX58 and RIG-1), cytokines (IL-1 $\beta$ and IL-18) and associated molecules (CASP-1 and PYCARD)were carried out using semi-quantitative SYBR Green Real Time PCR to identify the key inflammasomes mediating the Hepatitis E pathogenesis. Statistical analysis was performed using Mann-Whitney test. HEV positive patients presented with abdominal pain $(35.7 \%)$, yellow eyes $(35.7 \%)$, nausea $(35.7 \%)$, anorexia $(28.5 \%)$, fever $(28.5 \%)$, diarrhea $(28.5 \%)$, arthralgia (14.2\%), hepatomegaly (14.2\%), fatigue (14.2\%) and splenomegaly $(7.14 \%)$. Serum biochemical parameters shown ALT: $292.25 \pm 253.70$, AST: $260.62 \pm 180.04$, ALP: $230.5 \pm 143.6$ and Bilirubin (mg/dl) $3.39 \pm 1.28$. Relative gene expression in PBMCs of $14 \mathrm{AVH}$ and 11 healthy controls showed significant fold up-regulation of RIG-1 and IL-1 $\beta$ ( $5 \times$ and $14 \times$ respectively) with respective normalization values $(0.061 \pm 0.203$ Vs $0.002 \pm 0.002 ; P=0.026)$ and $(0.022 \pm 0.054 \mathrm{Vs} 0.001 \pm 0.007 ; P=0.005)$. Inflammasomes are very important components in HEV pathogenesis where RIG-1 appears to play a pivotal role through up-regulation of IL-1 $\beta$ cytokines genes. The phenotypic expression in terms of secretory cytokines in the extracellular milieu will reinforce this finding.

\section{Plant Virology}

\section{Oral Presentation}

\section{Structural and molecular insights into tospovirus-host interactions}

\section{H.R. Pappu, C. Olaya, S.V. Ramesh, and Y. Zhai}

Email ID for Correspondence: hrp@wsu.edu Department of Plant Pathology, Washington State University, Pullman, WA 99164, USA

Tospoviruses (family Bunyaviridae) cause economically important diseases in a wide range of crops. Tomato spotted wilt virus (TSWV) is the most prolific and cosmopolitan virus of the group. The genome of TSWV consists of three RNAs and the non- structural protein (NSs), coded by the S RNA, functions as a RNA silencing suppressor. Two conserved regions were identified in the NSs and mutational analysis showed that these motifs are critical for the suppressor activity. To better understand the structure-function relationships of the nucleocapsid gene $(\mathrm{N})$, and the NSs, we used 3D modeling algorithms, MULTICOM-CLUSTER, MULTICOMCONSTRUCT, MULTICOM-NOVEL, ITASSER, ROSETTA and CONFOLD to predict the secondary and tertiary structures of the $\mathrm{N}$ and the NSs proteins, and to determine the potential locations of several conserved amino acid motifs in these proteins. Using TSWV NSs as the reference sequence, nine conserved residues were identified among 27 tospoviral species and their locations within NSs were predicted. The TSWV-tomato interactions were studied at the transcriptome level using next generation sequencing. Virus specific small RNAs (vsiRNA) and their propensity for host mRNAs were determined. A subset of highly expressed viral vsiRNAs derived from the TSWV genome was analysed for their propensity to down regulate the tomato transcriptome using one TSWV resistant and one susceptible cultivar. Unique vsiRNAs were found to have potential cross-reacting capability with the tomato transcriptome. VsiRNAs were found to potentially target a gamut of host genes involved in basal cellular activities including enzymes, transcription factors, membrane transporters, and cytoskeletal proteins. The TSWV-tomato interaction at the sRNA interface points to the ability of tomato cultivars to overcome vsiRNA-mediated targeting of NBS-LRR class $R$ genes. Our findings suggest the prevalence of vsiRNA-induced RNA silencing of host transcriptome and provide a deeper understanding of the role of vsiRNAs in pathogenicity and in perturbing host machinery.

\section{Virus propagation in host: a trade off to host's fitness}

\section{Shelly Praveen}

Email ID for Correspondence: shellypraveen@hotmail.com Division of Biochemistry, Indian Agricultural Research Institute, New Delhi, India

The impact of viral infections on host fitness is a less studied phenomenon in plants. The manifestation of different symptoms in host plants during viral infections is likely to be, as one of trade off consequence. Since, host and virus have to co-exist, their interactions lead to mutualism. During virus propagation in the host cell, the change in cellular dynamics result in aberrations. Some of the viral proteins are crucial in altering cellular metabolism in favour of virus. Successful virus propagation in host requires, diversion of host resources towards viral multiplication needs and quenching of host defense. Interference of viral proteins in organelles specific biochemical events results in dysfunctional cellular machinery. While studying three different host virus combinations; papaya-papaya ringspot virus, tomato-groundnut bud necrosis and tomato-tomato leaf curl virus, cross talk of viral proteins with cellular pathways was observed. Localization of viral proteins in different cellular organelles signifies their possible role in regulating cellular metabolism. Proteosomal functions get affected in presence of viral protein (PRSVHcPro). The intensity of trade off in host fitness at infection and systemic site was observed during Groundnut bud necrosis viral infection, which can be explained in terms of stress signaling. The involvement of another viral protein (GBNV-NSs) in initiating programmed cell death may have role in developing necrotic symptoms. The possible role of viral protein (ToLCNDV-AC4) in interfering epigenetic modifications resulting in aberrant phenotype has been discussed. The intensity of trade off in host fitness while coping with viral infections is one of the determining factors of development of varying severity of symptoms.

\section{Role of quarantine in biosecuring crops from transboundary} plant viruses: the case study of quarantine of transgenic soybean germplasm

\section{Celia Chalam, AK Maurya, Chanda Priyadarshini,} Ruchi Sharma, Shashi Bhalla and SC Dubey

Email ID for Correspondence: celia.chalam@icar.gov.in; mailcelia@gmail.com

Division of Plant Quarantine, ICAR-National Bureau of Plant Genetic Resources, Pusa Campus, New Delhi, India-110 012

Soybean [Glycine max (L.) Merr.] is an important oilseed crop grown widely in India and is infected by 44 viruses under natural conditions, out of which, 30 are seed-transmitted. The germplasm of transgenic soybean with desirable traits is being imported into the country for various research purposes. The pest risk analysis revealed that 14 viruses are of quarantine significance for India, out of which, 12 are not reported from India and can be introduced through import of soybean seeds. This calls for stringent quarantine processing of 
imports. As per the Plant Quarantine (Regulation of Import into India) Order, 2003 (PQ Order, 2003), the ICAR-National Bureau of Plant Genetic Resources is empowered for quarantine processing of germplasm including transgenics imported for research purposes into the country. The PQ Order, 2003 requires Additional Declarations to be included in Phytosanitary Certificate for soybean seeds as free from viruses. During 1997-2017, a total of 366 seed samples of transgenic soybean from USA were imported and processed for quarantine clearance. These were grown in National Containment Facility of level-4 (CL-4) since its inception to ensure that no viable biological material/pollen/pathogen enters or leaves the facility during quarantine processing of transgenics. In addition, post-entry quarantine inspections of the transgenic soybean grown by indenters are also undertaken. The plants of the accessions showing virus-like symptoms and representative healthy-looking samples were further subjected to electron microscopy, ELISA and RT-PCR. The interceptions include nine destructive viruses including five viruses yet not reported from India viz., Bean mild mosaic virus, Cherry leaf roll virus, Cowpea severe mosaic virus, Raspberry ringspot virus and Tomato ringspot virus and one virus i.e., Arabis mosaic virus, not known to occur on soybean in India, hence, quarantine significance for India. The infected plants were uprooted and incinerated. The harvest from only virus-free plants was released for use in crop improvement programmes. The present findings highlight the importance of pest risk analysis and adopting the reliable techniques for virus detection in minimizing/eliminating the risk of introducing destructive exotic viruses, along with imported seeds. Since viruses are not easy to be controlled by chemical or physical means, their detection in imported material and their interception will ensure biosecurity from plant viruses through quarantine.

Epigenetic regulation of geminivirus transcription by replication initiator protein

\section{Nirbhay K Kushwaha, Mansi Bhardwaj and Supriya Chakraborty}

Email ID for Correspondence: supriyachakrasls@yahoo.com Molecular Virology Laboratory, School of Life Sciences, Jawaharlal Nehru University, New Delhi -110 067

Geminiviruses, largest group of pant viruses, consists of ssDNA genome and require the replication initiator protein (Rep) for replication to occur. In the nucleus of infected cells, in association with histone proteins the viral genome forms minichromosome like structure with 9-12 nucleosomes that act as potential substrate for histone post translational modifications. However, molecular mechanism of recruitment of post translational modification machineries onto the viral promoter was not known until present study. Here we demonstrate Rep mediated recruitment of monoubiquitination machineries onto the viral promoter which promotes transcription of the viral genome. We isolated $N b U B C 2$ (E2) and NbHUB1 (E3) two enzymes of monoubiquitination machinery from Nicotiana benthamiana a permissive host and implicated its role in Chilli leaf curl virus (ChiLCV) pathogenesis. Silencing of $N b U B C 2$ and $N b H U B 1$ in $N$. benthamiana leads to attenuated symptoms accompanied by reduced H2B-ub, H3K4-me3 and RNA polII occupancy on the ChiLCV promoter leading to reduced viral promoter activity. Our study has shown interaction of Rep with NbUBC2 and NbHUB1. Furthermore localization and co-localization studies suggested redirection of NbUBC2 from cytoplasm to nucleus. Interestingly viral Rep protein is organized as 'beads on string' like structure in presence of NbHUB1 and ChiLCV genome. Specific binding of Rep with viral genome facilitates recruitment of monoubiquitination machinery on viral promoter. Taken together the current study demonstrated novel role of geminivirus Rep protein in the epigenetic regulation of viral transcription. The identification of cellular and viral proteins necessary for stimulation of transcription of viral genes will be useful for identification of many regulatory circuits in the future.

\section{Emerging plant viruses: a threat for future}

\section{Krishna Reddy}

Email ID for Correspondence: mkreddy@iihr.res.in, mkreddy60@gmail.com

Division of Plant Pathology, ICAR-Indian Institute of Horticultural Research, Hessaraghatta Lake PO, Bengaluru-560089

During last decades, worldwide agriculture production has been compromised by a series of epidemics caused by new viruses that spilled over from reservoir species or by new variants of classic viruses that show new pathogenic and epidemiological properties. Plant virus diseases are emerging as a serious constraint in improving productivity and a limiting factor in many crop production systems in India. Plant viruses are a significant threat to global food security, and the majority of viruses that infect plants are naturally spread through three main transmission pathways: pollen, seed, and vector. Molecular ecology, within 10 years, has unravelled the factors responsible for the emergence of several of the economically most important tropical plant viruses. Emerging viruses have appeared in India through different routes or mechanisms, and their effects on hosts have also varied. Most of these viruses belong to tobamovirus (1.8\%), polerovirus $(1.7 \%)$, closterovirus $(3.9 \%)$, ilarvirus $(4.0 \%)$, badnavirus $(6.1 \%)$, tospovirus $(6.6 \%)$, cucumovirus $(7.0 \%)$, potyvirus $(22.7 \%)$ and begomovirus $46.2 \%$ ). Globalization and the development of international trade in agricultural and horticultural produce are breaking down traditional geographical barriers to pathogen movement. As trade becomes more international, the potential for importation of nonindigenous plant viruses grows significantly. In addition, changing climate conditions can contribute to a successful spread of newly introduced viruses or their vectors and establishment of these organisms in areas that were previously unfavourable. Major contributory factors for the emergence and spread of new viruses include evolution of variants of viruses, changes in vector biology, changes in the cropping systems, introduction of new crops, the movement of infected planting materials and introduction of susceptible host genes through exchange of germplasm. A large range of mechanisms most unsuspected until recently, were involved: recombination and synergism between virus species, new vector biotypes, genome integration of the virus, host adaptation, and long-distance dispersal. A complex chain of molecular and ecological events resulted in novel virus-vector-plant-environment interactions that led to virus emergence. It invariably involved a major agricultural change: crop introduction, cultural intensification, germplasm movement, and new genotypes. A current challenge is now to complement the analysis of the causes by an assessment of the risks of emergence. In light of growing international concern for crop biosecurity, new, emerging and threatening plant viral diseases are receiving greater attention. Improved and coordinated viral disease surveillance systems, enhanced global response capacity, and education and training of a multidisciplinary workforce are needed to adequately address new and emerging viral diseases will be discussed. 
Exploring the defence and counter-defence strategies in plantvirus interactions

\section{Ravi Kant, Madhvi Naresh, Fauzia Zarreen, Rashmi Rishishwar and Indranil Dasgupta}

Email ID for Correspondence: indranil58@yahoo.co.in Department of Plant Molecular Biology, University of Delhi South Campus, Benito Juarez Road, New Delhi-110021

Following virus infection, plants launch a defence response, named RNA-interference (RNAi), which is targeted to specifically degrade viral transcripts and bring about epigenetic changes in viral DNA. Viruses, on their part, suppress the above defence response using viral proteins, which are termed RNAi suppressors. We have explored this interplay in two DNA viruses; one, a member of family Caulimoviridae, named Rice tungro bacilliform virus (RTBV) and another, of family Geminiviridae, namely, Bhendi yellow vein mosaic virus (BYVMV). RTBV is part of a two-component virus system responsible for tungro disease in rice, prevalent in South and Southeast Asia, whereas BYVMV, along with a satellite DNA causes the yellow vein disease of okra, the most important constraint to okra porduction in the tropics. Using small RNA analysis of RTBV-infected rice plants, it was determined that the viral transcripts were targeted uniformly for degradation and to predict which of the components of the RNAi partricipated in the process. To check the potentail RNAi suppressor activities, tobacco plants, where transgenically expressed GFP gene was silenced using a gfp hairpin construct, were checked for the extent of gfp silencing following RTBV gene co-infiltration. Two RTBV gene products showed such RNAi suppressor activities. Interestingly, when checked for such activity in emerging leaves, one of them showed RNAi enhancement as well. This could be a strategy of the virus to bring about a basal level of infection to ensure long-term survival of the host plant. One of them interacted with the plant RNAi machinery in vivo. This indicated the probable mechanism of action of this novel supprerssor. A new gene of BYVMV was also found to possess RNAi suppressor activity using the tobacco system. Investigations are ongoing to determine its interacting partners.

\section{Tomato leaf curl New Delhi virus-emergence as invasive pathogen}

\section{V.G. Malathi, P. Renukadevi}

Email ID for Correspondence: vgmalathi@rediffmail.com Department of Plant Pathology, Tamil Nadu Agricultural University, Coimbatore-641003

The virus species Tomato leaf curl New Delhi virus (ToLCNDV) a bipartite begomovirus characterized from New Delhi and Lucknow in 1995 is the emerging devastating pathogen infecting wide range of solanaceuous and cucurbitaceous vegetable crops. ToLCNDV is the only begomovirus which has no trans-boundary limitations in movement across different countries in Asia and Europe. At present, it appears that ToLCNDV is well spread in all these Asian, North African and Southern Europe countries. Typical symptoms of disease are curling, puckering of leaves, veinal yellowing, stunting, excessive branching, pale yellowing to deep yellowing of leaves. In severely stunted plants, flowers may drop off. In some genotypes, green vein banding, twisting, green enation are also seen on the under surface of the leaf. ToLCNDV infection in okra has been identified in 2004, in majority of cucurbitaceous hosts between 2004 and 2007 and later, in other vegetables during 2009-2012. It is interesting to note that in Southern India ToLCNDV is still confined to cucurbits and has not yet been met with in tomato. The sequence identity of ToLCNDV with the monopartite begomoviruses ranged from 69 to $73 \%$. Interestingly with tomato leaf curl Rajasthan virus it was $86 \%$. According to ICTV, there are only two strains of ToLCNDV. An analysis performed including all the isolates (the present study) clearly showed that there is no association between host species and clustering of ToLCNDV isolates. The ToLCNDV isolates which showed $<90 \%$ identity branched off independently akin to other monopartite viruses. ToLCNDV though bipartite was found associated with both alpha and betasatellites. Plants co-inoculated with betasatellites showed enhanced symptom severity in both $N$. benthamiana and tomato and increase in helper viral DNA A and DNA B levels. ToLCNDV DNA $\mathrm{B}$ is an interesting molecule which shares high identity with DNA B of at least three viruses, ToLCGV, BYVMV and PepLCLaV. In all these cases identity in DNA A is only $65 \%$ but DNA B shows 85-89\% identity with ToLCNDV- DNA B. Infectivity and transreplication of these DNA B by ToLCNDV DNA A resulted in severe symptom expression. It is possible that these monopartite begomoviruses occurred in mixed infection with ToLCNDV and captured DNA B. Expansion in host range perhaps could be due to capturing of satellite DNAs and the ability of DNA B to complement different DNA A. Added to these features, in our recent studies we found seed borne nature of ToLCNDV in cucurbitaceous hosts like ash gourd and chayote. Importance of these observations in understanding emergence of ToLCNDV as invasive pathogen will be discussed.

\section{Piper yellow mottle virus: characterization and management}

\section{A. I. Bhat ${ }^{1}$, V. Srinivasan ${ }^{2}$, Anke Gowda ${ }^{2}$, K. S. Krishnamurthy ${ }^{2}$} and C. N. Biju ${ }^{1}$

Email ID for Correspondence: aib65@yahoo.co.in

${ }^{1}$ Division of Crop Protection, ${ }^{2}$ Division of Crop Production, ICARIndian Institute of Spices Research, Kozhikode 673012, Kerala

A viral disease also known as stunted disease, has been reported in black pepper growing in countries such as Brazil, India, Indonesia, Malaysia, Philippines, Sri Lanka and Thailand. In India, high incidence and severity of the disease was reported from black pepper plantations located at high altitudes such as Idukki and Wayanad districts of Kerala; Kodagu and Hassan district of Karnataka. Mosaic, mottling and formation of malformed as well as small sized leaves are the most obvious symptoms for identifying the disease in the field. In severe cases, the leaves become narrow; internodes of vines become abnormally short, leading to stunting of whole vine. The causal virus associated with the disease was identified as Piper yellow mottle virus (PYMoV) (genus: Badnavirus). The major means of spread of the virus is through the use of infected stem cuttings, which are used widely as planting materials. In addition, the virus can also spread through mealybugs and seeds. In view of the systemic nature and lack of chemicals to control the virus, identification and propagation of virus-free planting materials of elite genotypes is the most viable and important strategy in disease management. In this direction, we have developed nucleic acid-based sensitive methods like Polymerase chain reaction (PCR), real-time PCR and loop-mediated isothermal amplification (LAMP) and validated the detection of PYMoV in black pepper plants. Protocols for elimination of the virus from infected black pepper through somatic embryogenesis and meristem tip culture have also been developed. Our recent studies have indicated that severity of the disease increases when virus infected plants are subjected to abiotic stresses such as temperature and nutrients. In view of this we have developed a package involving application of site specific nutrients based on the soil test and providing irrigation during summer for the effective management of the disease infected black pepper plantations. 
Variability studies of pigeonpea sterility mosaic emaraviruses in India provides evidence of segment reassortment and recombination

\section{Basavaprabhu L. Patil, Meenakshi Dangwal and Ritesh Mishra}

Email ID for Correspondence: blpati12046@gmail.com, basavaprabhu.patil@icar.gov.in

ICAR-National Research Centre on Plant Biotechnology, Pusa, New Delhi, 110012, India

Sterility mosaic disease (SMD) of pigeonpea is a serious constraint for cultivation of pigeonpea in India and other South Asian countries. SMD of pigeonpea is associated with two distinct emaravirus species, Pigeonpea sterility mosaic virus 1 (PPSMV-1) and Pigeonpea sterility mosaic virus 2 (PPSMV-2), with genomes consisting of five and six negative-sense RNA segments, respectively. The recently published genome sequences of both PPSMV-1 and PPSMV-2 are from a single location, Patancheru from the state of Telangana in India. However, here we present the first report of sequence variability among 23 isolates of PPSMV-1 and PPSMV-2, collected from ten locations representing six states of India. Both PPSMV-1 and PPSMV-2 are shown to be present across India and to exhibit considerable sequence variability. Variability of RNA3 sequences was higher than the RNA4 sequences for both PPSMV-1 and PPSMV-2. Additionally, the sixth RNA segment (RNA6), previously reported to be associated with only PPSMV-2, is also associated with isolates of PPSMV-1. Multiplex reverse transcription PCR (RT-PCR) analyses show that PPSMV-1 and PPSMV-2 frequently occur as mixed infections. Further sequence analyses indicated the presence of recombination and reassortment of RNA4 between isolates of PPSMV-1 and PPSMV-2.

\section{Evolution of diverse papaya ringspot virus population: hosts} and geo-climatic locations are the key strikers

\section{Basavaraj $^{1}$, Ashwini Kumar ${ }^{2}$, Parameshwari, B. ${ }^{3}$, Jai Prakash ${ }^{4}$, Rakesh Kumar Jain ${ }^{1}$}

Email ID for Correspondence: rajayb@gmail.com ${ }^{1}$ Advance Centre for Plant Virology, Division of Plant Pathology, ICAR-Indian Agricultural Research Institute, New Delhi-110012, India; ${ }^{2}$ Department of Agicultural Biotechnology, Sardar Vallabh Bhai Patel University of Agriculture and Technology, Modipuram, Meerut, Uttar Pradesh-250 110, India; ${ }^{3}$ ICAR-Sugarcane Breeding Institute, Regional Centre, Karnal-132 001, India; ${ }^{4}$ Division of Fruit Science and Technology, ICAR-Indian Agricultural Research Institute, New Delhi-110012, India

Papaya ringspot virus (PRSV) encodes 10 proteins, of which only the coat protein $(\mathrm{CP})$ has been studied extensively from papaya-originated PRSV-P isolates. In this study, besides CP, the helper component proteinase (HC-pro) and nuclear inclusion protein-a (NIapro) coding regions from 19 PRSV-P and $-\mathrm{W}$ isolates originating from different hosts and geo-climatic locations were analyzed. Unlike CP (846-876 bp), both HC-pro (1370 bp) and NIa-pro (723 bp) did not exhibit heterogeneity in length, but, in sequence identity. HC-pro and NIa-pro coding regions exhibited up to 5 and $9 \%$ aa diversity respectively within Indian population and up to 6 and 10\% when compared with rest of the world. In contrast, the $\mathrm{CP}$ was highly variable (up to $20 \%$ aa diversity) within Indian population and rest of the world. Surprisingly, the highest variability in sequence length (846-876 bp) and identities (up to $20 \%$ aa diversity) was noticed among the cucurbit-originated Indian PRSV-P isolates, representing Warm semi-arid (WSA) climate. However, the papaya-originated-
PRSV-P isolates representing Oceanic $(O c)$ climate were quite conserved and less variable (up to 5\% aa diversity). The phylogenetic clustering of PRSV isolates was largely based on geo-climatic locations and hosts of their origin. Three recombinant isolates each based on NIa-pro and CP were detected revealing that they possessed cucurbit-originated PRSV (either -P or -W) isolates as one of their major/minor parents. In relation to the recombination events observed, all the three protein coding sequences were used further to generate the patterns of natural selection at codon level. It was observed that $\mathrm{CP}$ is under highest selection pressure as six sites were detected under positive selection, followed by NIa-pro with one site, whereas, HC-pro with none. These results indicated that the genetic recombination between the isolates of different host origin and the selection pressure by host and geo-climate are the significant contributors for the evolution of diverse PRSV population.

Sequence diversity of Begomovirus infecting papaya (Carica Papaya) and in silico prediction of papaya encoded microRNAs targets

\section{Abhinav Kumar}

Email ID for Correspondence: abhinav.kumar@iilmcet.ac.in Dept of Biotechnology, IILM college of Engineering and technology, Greater Noida-201306

Leaf curl disease of papaya is a dreaded disease caused by Begomovirus, family Geminiviridae. The leaf curl disease of papaya is transmitted by whitefly (Bemisia tabaci), and is single stranded monopartite in nature; however bipartite genome is also reported. The disease is characterized by downward curling and cupping of leaves followed by vein thickening. Every year, it causes tremendous loss to the papaya growing areas in India. A comparative study considering complete nucleotide sequence and the betasatellite sequence showed various groups of this virus. This study throws light on the similarity and dissimilarity between the different isolates of papaya leaf curl virus that has infected papaya plant in various regions of India and sub continents. MicroRNAs (miRNAs) belong to class of endogenous small RNAs which suppress expression of genes following cleavage or translational inhibition of target messenger RNAs. They are involved in a number of plant processes such as development, biotic and abiotic stresses. In this study, in silico approach is employed, high scoring miRNA-target pairs satisfying rules of minimum free energy and maximum complementarities were selected to investigate, if they have the potential to bind the genome of Begomovirus infecting papaya. The results indicated while cpa-miR $8139 \mathrm{a} / \mathrm{b} / \mathrm{c} / \mathrm{d} / \mathrm{e}$ can target all viral genes; cpa-miR166d can target $\mathrm{CP}$ and $\mathrm{AC} 1$ and AC4 gene. This study shows predicted papaya miRNAs which have the potential to target different genes, including AC4 region, which involve in gene silencing suppression.

\section{Diversity in plant virus populations and emerging challenges in India}

\section{K Subramanya Sastry}

Email ID for Correspondence: ksubramanyasastry1944@gmail.com; kssastry1944@yahoo.com

Retired Principal Scientist (Plant Pathology), Bangalore

Plant virus diversity is generally recorded based on the changes in the nucleotide and amino acid sequences of the virus and changes in symptom phenotype or symptom severity (either mild or severe), host range, vector behavior etc. However, in some cases, virus may infect 
plants without causing symptoms. When changes in severity of symptoms, host range, virus nucleotide and amino acid composition are significantly different as compared to previously recognized plant virus species, a new virus species is proposed as per norms of ICTV. The ICTV is reporting the increasing number of plant virus species year after year and in the 10th report of ICTV classification (2017), the number of plant virus and viroid species are listed nearly 1390. In this presentation, the information on the newly identified and recognized plant viruses are discussed. The species of Tomato leaf curl virus, Tomato yellow leaf curl virus, Cassava mosaic virus, Cotton leaf curl virus, Okra yellow vein virus (transmitted by whitefly vector); Maize streak virus (Leafhopper vector); Barley yellow dwarf virus, Banana bunchy top virus, Banana streak virus (aphid vectors) are listed as having number of new strains and some newly identified virus species, based primarily on levels of nucleotide identity (as other properties are same). The ICTV in recent years is recognizing and classifying the viruses solely based on metagenomic sequence data and are incorporated into the ICTV approved list (2017), based on the sequence relationships to existing taxa, even though the biological information of the plant viruses is lacking. Large numbers of virus species of the genus Begomovirus have been reported. For example, in India, tomato leaf curl New Delhi virus has very wide host range and causes economic yield losses. Most important factor for begomoviruses spread is the whitefly (Bemisia tabaci) which exists as number of biotypes due to genetic changes induced by ecological pressures which may result in wider spread of begomoviruses and concomitant threat to agriculture. The diversity of reported RNA viruses has also been gradually increasing for the past two decades. Further, thrips transmitted tospoviruses and aphid transmitted viruses diversity has also been recorded by the 10th ICTV. Recombination within and between species of other viruses with RNA genomes has also been frequently documented, especially among potyviruses. It is also established that diversity is also recorded in insect vectors, by which further increased spread of the plant viruses will take place. In order to achieve food security for the rapidly growing human population, it is desirable to use the latest molecular diagnostic techniques and develop suitable integrated management measures against the well-established and the newly emerging viruses causing heavy yield losses.

\section{Plant virus genome based vectors for genome engineering of crop} plants

\section{Anirban Roy, A. Abdul Kader Jailani, Bikash Mandal}

Email ID for Correspondence: anirbanroy75@yahoo.com Advanced Centre for Plant Virology, Division of Plant Pathology, Indian Agricultural Research Institute, New Delhi - 110012

Genome engineering (GE) have recently evolved as promising technology for improvement of a wide range of organisms, including plants. CRISPR/Cas9 DNA editing system can accomplish genome engineering through target-specific mutagenesis by inducing double stranded DNA breaks at a specific location followed by an error-prone non-homologous end joining and thus knocking out the specific gene function. It has been demonstrated that the CRISPR/Cas9 system is able to achieve efficient genome editing in plants through either transient or transgenic means. It has been shown that genome of a plant virus can be modified in a way so that it can efficiently replicate in the plant without producing symptoms or producing mild symptom. Hence, such modified virus genome based replicon can be used as a vector to carry a gene/gene fragment and deliver transiently into plant. The advantage for plant virus vector based delivery of genome editing tools is of two folds, (1) being transient in nature it overcomes difficulties associated with transgenic development and (2) with the movement and rapid replication of the virus throughout the plant, the DNA construct, which are being delivered will also spreads throughout the plants. Recently, autonomously replicating virus-based vectors have been demonstrated as efficient means of delivering GE reagents in plants. Both DNA viruses (bean yellow dwarf virus, wheat dwarf virus and cabbage leaf curl virus) and RNA virus (Tobacco rattle virus) have demonstrated efficient gene targeting frequencies in model plants (Nicotiana benthamiana) and crops (potato, tomato, rice, and wheat). We have developed croton yellow vein mosaic virus based replicon which can express a foreign gene in plant. Such vector is being employed to deliver transiently a Cas9 cassette and a gRNA construct against phytoene desaturase gene of host to evaluate their efficacy in genome editing of solanaceous plants.

Rapid detection of Sri Lankan cassava mosaic virus through loopmediated isothermal amplification assay

\section{Arutselvan R, T. Makeshkumar}

Email ID for Correspondence: rarutselvan@gmail.com ICAR- Central Tuber Crops Research Institute, Sreekariyam, Thiruvananthapuram, Kerala 695017

Loop-mediated isothermal amplification (LAMP) is a technique which can be very effective in detecting viruses of DNA genome. A one step LAMP assay was developed for detection of Sri Lankan cassava mosaic virus (SLCMV). A set of six primers were designed, based on the Coat protein gene sequence of the SLCMV virus. The assay was optimised to amplify SLCMV DNA under isothermal condition at $63{ }^{\circ} \mathrm{C}$ for $60 \mathrm{~min}$. LAMP amplification products were detected by visual examination using Hydroxynaphthol blue nucleic acid stain and had a ladder-like appearance on a $2 \%$ agarose gel. The detection limit of the LAMP assay was found to be 100 times sensitive than conventional PCR method. The assay was evaluated using diseased samples and the results indicated the reliability and simplicity of the assay as a quick field diagnostic tool for SLCMV. This assay is technically advantageous because it can all be done quickly in one step. In contrast with conventional PCR and real-time PCR assays, no need for expensive thermocycling equipment which makes LAMP much more efficient and cost effective.

\section{Rolling circle amplification (RCA)-PCR assay is more effective than traditional PCR for detection of begomoviruses infecting greengram (Vigna radiata)}

\section{B. V. Bhaskara Reddy, Chaithanya, B. H, Prasanthi, L}

Email ID for Correspondence: bvbreddy68@gmail.com Regional Agricultural Research Station, Acharya N G Ranga Agricultural University, Tirupati-517502

Rapid and accurate detection of the causal virus in plants and vector is a prerequisite for monitoring plant virus epidemics and specific diagnosis of causal virus associated with disease. The yellow mosaic disease of greengram in Andhra Pradesh is caused by two species of begomoviruses (MYMV and MYMIV). Hence there is a need to develop a reliable and effective technique to detect a causal virus associated with yellow mosaic disease of greengram. In the present study nine symptomless greengram samples were subjected to traditional PCR with virus specific primers to DNA-A and DNA-B components of Mungbean yellow mosaic virus (MYMV) and DNA-A of Mungbean yellow mosaic India virus (MYMIV). Out of nine samples tested, three samples were showed positive results with MYMV-A specific primers and two samples were amplified with 
MYMV-B specific primers. PCR amplification with MYMIV-A specific primers yielded five samples positive. The RCA-PCR techniques involves amplification of circular single stranded viral DNA with $\$ 29$ DNA polymerase followed by PCR with specific primers. The samples which are shown negative in PCR were subjected to RCA-PCR assay for further confirmation. In RCA-PCR assay all nine samples were found positive with DNA-A and DNA-B of MYMV and DNA-A of MYMIV. Hence it is proved that RCA-PCR assay is more sensitive than traditional PCR assay to detect begomoviruses associated yellow mosaic disease of greengram.

Detection of endogenous and episomal banana streak viruses from local banana mats prevalent in North East India

Saurabh Kumar Dubey ${ }^{1}$, Y. Rupert Anand ${ }^{2}$, S. Monteshori ${ }^{2}$, Richa Rai ${ }^{1}$, V. K. Baranwal ${ }^{1}$ and Susheel Kumar Sharma ${ }^{2}$

Email ID for Correspondence: sauravdubey12179@gmail.com ${ }^{1}$ Advanced Centre for Plant Virology, Division of Plant Pathology, ICAR-Indian Agricultural Research institute, New Delhi-110012;

${ }^{2}$ ICAR Research Complex for NEH Region, Manipur Centre, Imphal795004

Banana streak disease is caused by a cryptic virus species complex of the genus Badnavirus in the family Caulimoviridae, collectively known as banana streak viruses (BSV). Virus exists in both episomal and endogenous forms. In the present study, surveys were carried out in different banana growing pockets of Manipur, Tripura and Nagaland. Infection of episomal BSV was detected using antigen coated plate trapped-ELISA (ACP-ELISA), duplex-immunocapture-PCR (DIC-PCR) and rolling circle amplification (RCA). ACP-ELISA and D-IC-PCR was performed with antibodies against BSMYV. Since banana infecting badnaviruses shows high level of serological heterogeneity and no serological cross-reactivity; the infection of other Badnavirus species (BSV species other than BSMYV) may go undetected. RCA amplifies the minichromosomal form of Badnavirus DNA in sequence independent manner, hence, results based on RCA were considered confirmatory. RCA coupled with restriction fragment length polymorphism (RCA-RFLP) yielded linear RCA monomers of the size $7.5-8.0 \mathrm{~kb}$ confirming their episomal origin. Out of 58 samples tested, 14 were found to be virus positive in RCA/RFLP for the infection of episomal BSVs. These samples originated from Leimatak, (Bishnupur District, Manipur), Longmai, (Noney District, Manipur) and Sovema and Jharnapani (Dimapur District, Nagaland). The viral identity of RCA-RFLP fragments was confirmed by sequencing. Presence of endogenous BSV sequences was confirmed using allele specific eBSV primer pairs in normal PCR. All the tested samples gave a PCR amplicon of $580 \mathrm{bp}$ and $600 \mathrm{bp}$ (RT/RNase H region of viral genome) indicating the presence of endogenous Badnavirus sequences. The sequences of representative endogenous Badnavirus showed genetic similarity to the Melicytus ramiflorus isolate ramWC5A endogenous virus Badnavirus, thus representing prevalence of unique endogenous florendoviruses in the local banana mats of NE India. Present study conclusively proved widespread occurrence of endogenous and episomal badnaviruses infecting local banana mats in NE India.
In-silico interaction of RNA silencing suppressors of velvet bean severe mosaic virus with DICER domains

\section{Aruna Jangid, Vanita Chandel, Ashish Srivastava and Narayan Rishi}

Email ID for Correspondence: anu_jangid25@yahoo.in Amity Institute of Virology and Immunology, Amity University, Noida, India

Velvet bean severe mosaic virus (VbSMV), a bipartite begomovirus causes severe mosaic, downward curling of the leaves, stunting of the plants in velvet beans (Mucuna pruriens). VbSMV contains two potential viral suppressors of RNA silencing, AC2 and AV2. To understand the mechanism of suppression of host defence and interaction of VSRs with host DICER, the in silico interaction study was performed. Evidence of interaction of the AC2 protein with the type III restriction enzyme domain, helicase superfamily c-terminal domain and transcription repair coupling factor, whereas AV2 protein with ribonuclease III C terminal domain, ERCC4-related helicase domain, ribonuclease III domain, Dicer dimerization domain, type III restriction enzyme RES subunit and transcription repair coupling factor were found. This is the first in silico evidence of interaction of any begomoviral VSRs (viral suppressors of RNA silencing) with Dicer of host.

\section{Molecular characterization of Taro bacilliform virus infecting} taro (Colocasia esculenta) in India

Aarthy, M.B., Arutselvan, R., Asha Devi, A., Jeeva, M.L. Makeshkumar, T

Email ID for Correspondence: makeshkumar.t@icar.gov.in; makeshctcri@gmail.com

ICAR-Central Tuber Crops Research Institute, Thiruvananthapuram, Kerala

Taro is one of the world's oldest food crops maintained by farmers for millennia and it has continued to spread throughout the world, as an important crop especially in Asia, Pacific, Africa and the Caribbean. Viral pathogens are one of the important factors threatening the production of this vegetatively propagated crop. Taro viruses are poorly characterized, which is a hindrance for safe movement of germplasm. The aim of this study was to detect the Taro bacilliform virus infecting taro and characterize the virus at molecular level. During the study 55 samples from different fields of ICAR-CTCRI were selected to identify the different symptoms of Taro bacilliform virus $(\mathrm{TaBV})$ infection. The symptoms and occurrence of TaBV could not be correlated even though the samples either showed different types of symptoms or asymptomatic. Molecular analysis with $\mathrm{Bad}$ navirus specific primers and further confirmation with PNG Badna F/R revealed the presence of Taro bacilliform $\mathrm{CH}$ virus (TaBCHV newly described virus in taro). Sequence and phylogenetic analysis confirmed that the sequences were more closely related to TaBCHV and exhibited $100 \%$ similarity to TaBCHV isolates TaBCHV-1 and TaBCHV-2. From the seven pair of primers, specifically designed in order to characterize the whole genome of Taro bacilliform $\mathrm{CH}$ virus, three pair of primers were used to successfully characterize the virus partially. Sequence and phylogenetic analysis confirmed the close association the virus with TaBCHV -1 and 2. 
Molecular analysis of Mungbean yellow mosaic India virus (MYMIV) Infecting Soybean in MP, India

\author{
Neeraj Verma, Jyoti Pandey \\ Email ID for Correspondence: neerajverma.2008@yahoo.com \\ Dept. of Agricultural Science AKS University, Satna (M.P.)
}

Yellow mosaic disease is a serious problem of soybean crop in MP India. It is one of the most important viral disease infecting soybean which causes severe yield reduction and economic loss. Soybean (Glycine $\max$ L. Merril) is one of the world's most important seed legume, contributing $25 \%$ of the global edible oil, about two-thirds of the world's protein concentrate for livestock feeding. Partial coat protein (CP) gene of Mungbean yellow mosaic India virus (MYMIV) infecting soybean in MP, India was analyzed to study molecular diversity using gene specific primers. Out of 10 samples, 08 samples were found to be positive for MYMIV, while all were negative for Mungbean yellow mosaic virus (MYMV). The nucleotide and amino acid homology of 93-100 and 99-100\%, respectively of MYMIV from MP, India was observed with other published sequences in GenBank. To distinguish MYMIV and MYMV, PCR based assay was found to be an effective method.

\section{On-site detection of plant viruses with special reference to banana viruses using lateral-flow devices}

\section{Ramasamy Selvarajan}

Email ID for Correspondence: selvarajanr@gmail.com/

Selvarajan.R@icar.gov.in

ICAR-National Research Centre for Banana, Tiruchirapalli,

Tamilnadu, India -620102

Plant viruses cause serious diseases that lead to significant production and economic losses in the agriculture and horticulture industries worldwide. Bananas are affected by four knowns, relatively wellcharacterized viruses such as banana bunchy top virus, banana streak virus, cucumber mosaic virus and banana bract mosaic virus. Currently, detection of viruses in banana is accomplished by ELISA and PCR. However, these methods are time-consuming, special equipment's are required, and qualified personnel are needed which limits the wider adoption. For routine monitoring of viruses in plants, a simple, reliable, highly specific, and sensitive rapid method to be used on-site by the ordinary user without the need of exceptional skills and equipment's was an urgent need. One-step lateral-flow tests have been developed for the on-site detection and identification of several plant viruses. They utilize specific monoclonal and polyclonal antibodies in an immunochromatographic format, incorporating antibody-bound nano-gold or latex particles. These tests procedures are user-friendly to allow disease diagnosis in the field within 2-5 min. At present, Lateral Flow immune-strips assays are not available, for on-site or infield use by the stakeholders of banana industry. We have developed lateral-flow devices for two banana viruses namely BBrMV and CMV. These tests are highly accurate, when compared with traditional microplate enzyme-linked immunosorbent assays. In this talk, the development of on-site detection tools for the plant viruses with more emphasis on banana viruses and other important clonally propagated horticultural crops shall be presented. The present status and the future perspectives of dip-stick technology for the virus disease diagnostics will be discussed in detail.
One step multiplex RT-PCR for simultaneous amplification of tripartite genome of Prunus necrotic ringspot virus

\section{Md Salik Noorani, Jawaid A. Khan}

Email ID for Correspondence: saliknoorani@gmail.com Plant virus Laboratory, Department of Biosciences, Jamia Millia Islamia (A Central University), New Delhi, India-110025

Prunus necrotic ringspot virus (PNRSV) belongs to the genus Ilarvirus of the family Bromorividae. It is a serious pathogen of the family Rosaceae and causes considerable economic losses to the stone fruit trees worldwide. The genome of PNRSV consists of a tripartite, positive-sense, single strand RNA genome. RNA1 and RNA2 are monocistronic and encode the replicase proteins, while RNA3 encodes two polypeptides, a putative movement protein and coat protein. In this study, a one step Multiplex RT-PCR was developed for the simultaneous amplification of all three fragments of the PNSRV genome in a single PCR tube. For these, the primers were designed after alignment of conserved region of the complete genome of PNRSV sequences available in the GenBank of NCBI. Fragments of approximately 3300, 2500 and 1900 bp were amplified successfully by one step Multiplex RT-PCR using designed primers from the infected plant samples. After amplification the identity of the fragments was confirmed by Nested and Hemi-nested RT-PCR and also by sequencing. To the best of our knowledge, this is the first report of amplification of all three fragments of PNRSV by Multiplex RT-PCR in one go.

\section{Structural studies on capsid protein of banana bunchy top virus}

\section{Sangita $V^{1}$, Sundaram $S^{1}$, Savithri $\mathbf{H S}^{2}$, Selvarajan $\mathbf{R}^{3}$}

Email ID for Correspondence: svenkataraman@ggn.amity.edu/ svenkataraman@ggn.amity.edu

${ }^{1}$ Amity University Haryana, Gurgaon 122413; ${ }^{2}$ Indian Institute of Science, Bengaluru, India - 560012; ${ }^{3}$ ICAR-National Research Centre for Banana, Tiruchirapalli - 620102

X-ray diffraction studies on single crystals of viruses enable visualization of the structures of intact virus particles at near-atomic resolution. These studies provide detailed information regarding the coat protein folding, capsid architecture, molecular interactions and plausible sites of receptor recognition. Such learning is pivotal in designing strategies for combating infections due to viruses in plants, animals and humans. Banana is the major staple food crop for approximately 400 million people. Of 114 million tonnes of banana that was produced globally in 2014, India accounted for 24.5 per cent of the global production. Bunchy Top disease of Banana is one of the most devastating diseases caused by Banana Bunchy Top Virus (BBTV) that results in a significant loss of yield, stunting and bunchy appearance of leaves. While many isolates of BBTV from various regions of India have been characterized by different groups, no structural study exists for this important virus.

In order to pursue structural studies, the coat protein gene from BBTV isolate of Hill Banana grown in lower Pulney Hills of Tamilnadu cloned in pET28a vector was expressed in BL21 (DE3) pLysS. Purification of the CP was done using Ni-NTA affinity chromatography. In vitro capsid assembly was studied using sucrose density gradient centrifugation which suggested that the CP does not assemble as virus like particle but stays as smaller oligomeric forms. Studies using dynamic light scattering (DLS) indicates that the purified protein is poly-dispersed represented majorly as tetramers. Studies using both homology modelling and ab initio structure determination gave useful insights into the probable fold of the $\mathrm{CP}$ 
and in silico capsid reconstruction aided understanding of the quaternary organization of subunits in the capsid.

\section{A potential alternate production platform for plant biologics: chikungunya vaccine candidates}

\author{
Sathishkumar. $\mathrm{R}^{1,2}$, Bala Murugan. $\mathrm{S}^{2}$, Gowtham. $\mathrm{I}^{1}$ and \\ Julian K-C $\mathrm{Ma}^{3}$
}

Email ID for Correspondence: rsathish@buc.edu.in

${ }^{1}$ Plant Molecular Farming Laboratory, DRDO-BU Centre for Life Sciences, Bharathiar University campus, Coimbatore - 641046. India; ${ }^{2}$ Plant Genetic Engineering Laboratory, Department of Biotechnology, Bharathiar University, Coimbatore - 641046, India; ${ }^{3}$ Institute of Infection and Immunity, St. George's, University of London, London SW17 ORE, United Kingdom

Conventional protein production hosts (bacterial/yeast/mammalian system) are well-recognized systems, that are currently in commercial practice. Final product obtained from these systems fail in commercialization aspects in the cost effectiveness and this made the researchers to look for the alternate cost-effective production platforms. Plant system is emerging now due to its several advantages like, glycosylation and established genetic and glycoengineering methods, adoptability, scalability and affordability. As a proof of concept study, we have demonstrated the expression of Chikungunya vaccine antigen candidates E1 and E2 in plant system. Super plant expression vector constructs like, pEAQ HT DEST and pTRAK was used in this study. The level of expression, glycosylation, purification and immunogenicity were analyzed and the results strongly suggests that the plants can be used for the production of CHIKV vaccine candidates. This study clearly proved that plant is a potential alternate expression system for the large-scale production of commercial proteins, a concept known as Plant Molecular Farming. This project was supported by UK-Indian Education and Research Initiative (UKIERI).

\section{Molecular characterization of sida yellow vein virus DNA A and sida leaf curl virus alphasatellite from Sida cordata, a commonly occurring weed in India}

\section{Sravya. $\mathbf{G}^{1}$, Bikash Mandal ${ }^{2}$, Selvarajan. $\mathbf{R}^{3}$, A. Swapna Geetanjali ${ }^{1}$}

Email ID for Correspondence: mithra.cuit@gmail.com ${ }^{1}$ Department of Genetic Engineering, SRM University, Kattankulattur, Tamil Nadu, India; ${ }^{2}$ Advanced Centre for Plant Virology, Division of Plant Pathology, Indian Agricultural Research Institute, New Delhi, India ${ }^{3}$ National Research Centre for Banana, Trichy, Tamil Nadu, India

Sida cordata is a commonly occurring weed in most of the economically important crop fields in India. In the present study we aimed to characterize the begomoviruses present in the weeds that are widely occurring in chilli fields. S. cordata leaf samples showing yellow vein symptoms were collected from the chilli fields of Thuvarankurichi near Trichy, Tamil Nadu during the month of August 2016. The genomic DNA isolated from the weed samples was subjected to Rolling Circle Amplification (RCA). The RCA product digested by BamHI and HindIII, resulted in 2.7 and $1.4 \mathrm{~kb}$ unit lengths of begomovirus fragments were cloned in pUC19 and sequenced. The sequence of DNA A clone contained $2754 \mathrm{bp}$ and it has shown $96 \%$ similarity with sida yellow vein Madurai virus. An alphasatellite containing $1388 \mathrm{bp}$, obtained from this sample has shown $91 \%$ similarity with sida leaf curl virus alphasatellite.
Polymerase chain reaction performed for the DNA sample by universal beta primers (Beta $\mathrm{F}$ and Beta $\mathrm{R}$ ) had given a positive amplicon of length $1.4 \mathrm{~kb}$, it was further cloned in TA vector and sent for sequencing. The study further provides the information about the association of betasatellite and sida leaf curl virus alphasatellite with sida yellow vein virus DNA A in $S$. cordata.

Identification and characterization of RNA silencing suppressor gene(s) of Sugarcane streak mosaic virus (SCSMV) through Agrobacterium mediated transient expression assay

\section{K. Bagyalakshmi ${ }^{1}$, K. Lakhsmi ${ }^{1}$, B. Parameswari ${ }^{2}$, V. G. Malathi ${ }^{3}$, R. Viswanathan ${ }^{1}$}

Email ID for Correspondence: rasaviswanathan@yahoo.co.in ${ }^{1}$ ICAR-Sugarcane Breeding Institute, Coimbatore 641007; ${ }^{2} \mathrm{SBI}$ Regional Centre, Karnal 132001; ${ }^{3}$ TNAU, Coimbatore 641003

RNA silencing is a natural defense mechanism against the entry of viral pathogens in any eukaryotic biological system. To counteract this defense, many viruses encode RNA silencing suppressors (RSS) which delay the defense response exerted against them. Sugarcane streak mosaic virus (SCSMV) is one of the causative viruses of mosaic in sugarcane in many Asian countries. We have sequenced and characterized complete genome sequences of the virus from India. Further studies were carried out to characterize RSS activity of P1 and HC-Pro genes of the virus. Initially signatures for the RSS activity were identified in P1 gene rather than HC-Pro through in silico analysis. However, their functional validation as RSS is not yet reported so far. Hence we have taken up detailed studies on the functional activity of P1 or HC-Pro cistrons in SCSMV as RSS through transient expression assays with respective gene constructs on the model plant Nicotiana tabacum. The plasmid constructs of both the cistrons were co-infiltrated with the reporter green fluorescent protein (GFP) and the suppressor activity was measured. Further, the expression level of the target mRNA was validated through semi quantitative and qRT-PCR. In the P1 co-infiltrated GFP leaves, suppression in the RNA silencing mechanism was observed that allowed a long term expression of GFP. However, GFP co-infiltrated with HCPro did not sustain the GFP expression level for a prolonged period and the expression level was close to GFP control. Our study concluded that unlike in other members of Potyviridae genera, P1 gene of SCSMV is playing the role of RNA silencing suppressor for the first time. Since P1 gene is directly countering host defence against SCSMV, targeting it through RNA silencing approach will be a viable strategy to develop mosaic resistant transgenic sugarcane.

\section{Mycoviral diversity in Rhizoctonia solani AG-1-IA infecting rice} in India

\section{Subha Das, Sampa Das}

Email ID for Correspondence: subhadas1981@gmail.com Division of Plant Biology, Bose Institute, Centenary Campus, P 1/12, C. I. T. Road, Scheme - VIIM, Kolkata 700054, West Bengal, India

Mycoviruses are omnipresent in different major fungal taxa. Genomes of majority of the mycoviruses consist of either double-stranded (ds) or single-stranded (ss) RNA. Mycoviruses usually infect their hosts in a cryptic manner, however some viruses have the ability to attenuate host virulence upon infection. Such viruses could be exploited as biocontrol agents to manage economically important crop diseases. Rhizoctonia solani AG-1-IA is the major pathogen of sheath blight diseases of rice. So far, no studies have provided a comprehensive 
picture of virus diversity in this pathogen. In this study, we extracted and profiled total dsRNAs from 32 AG-1-IA isolates collected from 10 different rice-growing states in India. All the isolates except one contained dsRNA elements. The number of dsRNA elements within individual isolates varied between one and seven, with approximate sizes ranging from 0.52 to $18.1 \mathrm{~kb}$. Total dsRNAs were randomly amplified, cloned and multiple clones were sequenced from eight individual AG-1-IA isolates with different dsRNA banding patterns. Sequences were assembled into contigs and subjected to BLASTX search, which identified the presence of viruses related to at least five known virus families: Endornaviridae, Partitiviridae, Megabirnaviridae, Narnaviridae and Rhabdoviridae. In addition, several unclassified dsRNA viruses were also detected. To characterise the virome in AG-1-IA by RNA-seq approach, dsRNA-rich fractions pooled from 20 different AG-1-IA isolates were sequenced using illumina NextSeq 500 platform, and sequence reads were assembled de novo. Assembled contigs sharing similarities with previously reported viruses showed affinity towards additional five virus families: Podoviridae, Retroviridae, Caulimoviridae, Microviridae and Reoviridae. A considerable number of contigs showed no BLASTX hit, suggesting the presence of novel viruses in the population that are yet to be characterised. This study for the first time provides an insight into the mycoviral diversity in $R$. solani AG-1-IA population infecting rice in India.

Geminivirus bidirectional promoters: a new toolkit to manipulate plant genomes

\section{Zainul A. Khan ${ }^{1,2}$, Jawaid A. Khan ${ }^{1}$}

Email ID for Correspondence: zainulbiotechnology@gmail.com ${ }^{1}$ Plant Virus Laboratory, Department of Biosciences, Jamia Millia Islamia (Central University), New Delhi-110025, India; ${ }^{2}$ Department of Plant Molecular Biology, University of Delhi South Campus, New Delhi-110021, India

Cotton leaf curl Kokhran virus- Burewala (CLCuKoV-Bu), previously known as Cotton leaf curl Burewala virus (CLCuBuV) belongs to the genus Begomovirus of family Geminiviridae and transmitted by whitefly (Bemisia tabaci). The intergenic region of begomoviruses possess bidirectional promoter. Bidirectional promoters possess the capability of expressing two genes simultaneously, thereby making them superior and functionally more efficient than the normal unidirectional promoter. In this study the bidirectional promoter representing complementary sense (Rep) and virion sense $(C P)$ gene of $\mathrm{CLCuBuV}$ were isolated and characterized. The transcription strength of these promoters was assayed both in stable and transient expression systems in tobacco as well as cotton plants, and was compared with $35 \mathrm{~S}$ promoter of Cauliflower mosaic virus (CaMV), which is more frequently used in plant genetic engineering. The bidirectional promoter of CLCuBuV and CaMV 35S promoter were fused with GFP and GUS reporter genes and was quantified using reverse transcription quantitative real-time PCR, fluorometric GUS assay, and confocal laser scanning microscopy (CLSM). The expression level of GUS driven by CLCuBuV complementary sense promoter in the transformed Nicotiana tabacum plants was shown to be four fold higher than that of CaMV 35S promoter, while the expression driven by $\mathrm{CLCuBuV}$ virion sense promoter was slightly lower than that of CaMV $35 \mathrm{~S}$ promoter. Further, the expression of GFP was monitored and compared in agro-infiltrated leaves of $N$. benthamiana, N. tabacum and Gossypium hirsutum plants using CLSM. CLCuBuV complementary sense promoter showed strong consistent transient expression in tobacco and cotton leaves as compared to CaMV $35 \mathrm{~S}$ promoter. In addition, the Rep::GFP promoter construct was used for the stable transformation of cotton and
Catharanthus roseus plants. The strong constitutive CLCuBuV bidirectional promoter developed in this study could be very useful for high level constitutive expression of transgenes in a wide variety of plant cells.

Virus indexing for certification of tissue culture raised banana using indigenously developed detection kits has attributed for the improved production of banana in India

\section{Velusamy Balasubramanian, Ramasamy Selvarajan}

Email ID for Correspondence: nvbalu75@gmail.com ICAR-National Research Centre for Banana, Tiruchirapalli, Tamilnadu, India -620102

Use of quality planting material in banana is very important for enhanced production and productivity. Viruses are the major problem. Four viruses viz., banana bunchy top virus, banana streak Mysore virus, cucumber mosaic virus and banana bract mosaic virus cause serious loss in production. Infected planting materials act as reservoir for viral pathogens and aggravate the incidence in field. National Certification System for Tissue Culture Raised Plants (NCS-TCP) implemented by Department of Biotechnology, Govt. of India has played a vital role in producing quality planting material. The success of certification is dependent on the availability of low cost diagnostics with more sensitivity and specificity. ICAR-NRC Banana has developed serological and PCR based detection techniques. In 2007, ICARNRCB has been accredited by DBT, Govt. of India. Till date, 220 million TC plants were certified from our facility. Both mother-plant and tissue-culture raised plants were tested against four banana infecting viruses. The percentage of positive plants for BBTV, BSMYV, CMV and BBrMV were 1.00, 0.38, 0.26 and 0.15 respectively. Based on our virus testing record the percentage of positives to virus in mother culture in TC banana has reduced from 12.76 to 0.01 significantly. The virus disease incidence has come down over the years after the implementation of certification programme. The banana production in India was 18.90 million tonnes in 2005 and has increased to 30.20 million tonnes in 2016. In this paper, the problems and prospects of virus indexing of TC banana in India is dealt in detail.

Zucchini yellow mosaic virus: an emerging threat to cucurbitaceous vegetables cultivation

\section{G. Karthikeyan ${ }^{1}$, S. K. Manoranjitham ${ }^{1}$ and K. Nagendran ${ }^{2}$}

Email ID for Correspondence: gandhikarthikeyan@tnau.ac.in ${ }^{1}$ Department of Plant Pathology, Centre for Plant Protection Studies, Tamil Nadu Agricultural University, Coimbatore 641003 Tamil Nadu; ${ }^{2}$ ICAR - Indian Institute of Vegetable Research, Varanasi 221 305 Uttar Pradesh

Zucchini yellow mosaic virus (ZYMV, genus Potyvirus, family Potyviridae) infects several cucurbitaceous vegetable crops and causes severe losses in their production worldwide. The infected plant exhibits severe mosaic, stunting with deformation of leaves and fruits. ZYMV is successfully transmitted to healthy plants by aphids and to some extent through seeds and mechanical means. A systematic field survey was conducted in major cucurbitaceous crops growing areas covering all the seven agroclimatic zones of Tamil Nadu. The samples were collected across thirteen cucurbitaceous crops. Initially samples were tested for the presence of ZYMV through DAS-ELISA using the commercial kit (DSMZ, Germany) and ZYMV—-specific immunostrips (Agdia, USA). Around 8 per cent of the samples were 
found to be infected with ZYMV. The total RNA was isolated from the symptomatic plant samples and was subjected to RT-PCR assay using the potyvirus universal primer pair (pNIbF1/pCPR1) spanning $3^{\prime}$ end of NIb region and $5^{\prime}$ portion of coat protein. The samples which were positive in DAS-ELISA and immunostrip assay, were found to be positive in RT-PCR also, by amplifying an amplicon of around $1100 \mathrm{bp}$. It was further confirmed through RT-PCR analysis by designing a gene specific primer pair (GKZYMV F: $5^{\prime}$-ATAGCTGAGACAGCACT- $3^{\prime}$ and GK ZYMV R: 5'CGGCAGCRAAAC GATAACCT- $3^{\prime}$ ) corresponding to the coat protein gene of ZYMV. All over again the results revealed that the samples (8\%) showed positive reaction in serological assays and RT-PCR with potyvirus universal primer was found to be positive for ZYMV coat protein. The infection of ZYMV was observed in pumpkin, cucumber and snake gourd. The distribution of the virus was seen in High Rainfall, Southern, Western and North-Eastern Zones of Tamil Nadu. Selective samples across the crops and zones were sequenced and analyzed. The sequence analysis indicated that the ZYMV isolates of Tamil Nadu have maximum identities with the Israel and Syrian isolates of ZYMV. The occurrence, distribution, genetic diversity and phylogeography will be discussed in the presentation.

\section{Poster Presenatation}

Detection and characterization of a virus causing leaf curl disease in Capsicum annuum in Himachal Pradesh

\section{Abhishek Bhardwaj, Saurabh Kulshrestha}

Email ID for Correspondence: abhishekbhardwaj1486@gmail.com Faculty of Applied Sciences and Biotechnology, Shoolini University of Biotechnology and Management Sciences, Solan-173212 (H.P.), India

Geminiviruses are one of the most destructive pathogens of Capsicum аппиит L. (bell pepper) causing considerable economic losses in capsicum production worldwide. Geminiviruses are characterized by a circular ssDNA genome and twinned-icosahedral particles. India contributes one-fourth of overall world production of capsicum, wherein Himachal Pradesh has recently been reported as second largest producer of capsicum after Karnataka in the country. The leaf curl disease in pepper is caused by Pepper/Chilli leaf curl virus (PepLCV/ChiLCV) and Tomato leaf curl virus (ToLCV) those belong to family Geminiviridae and genus Begomovirus. Hence, the aim was to detect and characterize the virus causing leaf curl disease in capsicum in Himachal. The characteristic symptoms of the disease includes curling, yellowing, puckering of leaves and stunted growth of the plants. 105 symptomatic leaf samples were collected from diseased plants from Solan, Sirmour, Shimla, Bilaspur and Kangra districts of the state and total DNA was extracted. Three sets of specific primers were used for PCR amplification of coat-protein (CP) gene (AV1), DNA-A and DNA-B components of the virus genome which further produced amplicons of $\sim 800 \mathrm{bp}, \sim 2600 \mathrm{bp}$ and $\sim$ $1500 \mathrm{bp}$ size respectively, in all infected samples confirming the presence of geminivirus infection. The amplicons for CP gene, DNAA and DNA-B segments obtained from SPUR1, SPC3, KPP4, BPP7 and SPP1 isolates were ligated into pGEM-T easy vector and transformed to DH5 $\alpha$ strain of E.coli. Upon sequencing the CP gene from the SPUR1 isolate was found to be 814 bp long showing $98 \%$ identity with the 'ToLCJV segment DNA- A complete sequence'; while the amplicon of DNA-B segment was found to be $1404 \mathrm{bp}$ long showing 90\% identity with 'ToLCBV betasatellite isolate Chhapra clone BamH 1-2 C1 gene'. The study provides molecular confirmation for the thriving geminivirus infection in capsicum crop around all of the five selected districts of Himachal Pradesh.
Vector and seed transmission study of bean common mosaic virus infecting cowpea [Vigna unguiculata L. (Walp.)] in Tamil Nadu

\author{
Abirami, $\mathbf{R}^{1}$, Renkudevi, $\mathbf{P}^{1}$, Malathi, V. $\mathbf{G}^{\mathbf{1}}$, Kumaresan, $D^{2}$, \\ Eraivan, $\mathbf{K}^{\mathbf{3}}$
}

Email ID for Correspondence: agriabirami@gmail.com

${ }^{1}$ Department of Plant Pathology, Center for Plant Protection Studies, Tamil Nadu Agricultural University, Coimbatore, Tamil Nadu, India641003; ${ }^{2}$ Department of Genetics and Plant Breeding, Hybrid Rice Evaluation Center, Gudalur, Tamil Nadu, India-643221; ${ }^{3}$ Department of Plant Pathology, Agricultural College and Research Institute, Madurai, Tamil Nadu, India-625104

Bean common mosaic virus (BCMV), a Potyvirus genus was found to be infecting cowpea in Tamil Nadu. BCMV was an ssRNA, long flexuous rods of about $10 \mathrm{~kb}$ size was transmitted by both vector and seeds. The vector associated with transmission of BCMV was morphologically identified as Aphis crassivora. Adult A. crassivora were collected from cowpea fields of TNAU, Coimbatore and reared on cowpea (Co (Cp-7)) for transmission studies. Different acquisition access periods (AAP) viz, $10,20,30,45,60$ and $120 \mathrm{~min}(\mathrm{~min})$ and different starvation periods (SP) viz, 0, 10, 30, 45, 60 and 120 min were carried out. Among different treatments of AAP, 45 min of AAP showed mosaic mottling symptom after 12 days with $20 \%$ transmission and in SP experiment maximum transmission was recorded in 120 min with $60 \%$ transmission followed by 33.3 and $40 \%$ transmission in 45 and 60 min respectively by producing mosaic mottling symptoms within 10-12 days at 45 min AAP. Seeds collected from BCMV infected cowpea field and BCMV sap inoculated plants exhibited seed transmission rate of 53 and $47 \%$ in 14 and 7 days respectively by producing mosaic mottling symptoms through grow-out test. Reverse Transcriptase Polymerase chain reaction (RT-PCR) using coat protein gene specific primer from 3' end of Nib to 3' end of CP gene of BCMV was performed for viruliferous aphids and symptomatic leaves of both vector transmission and grow-out test experiments which produced an amplicon size of $\sim 850 \mathrm{bp}$ confirming the presence of BCMV. To our knowledge this is the first report of aphid and seed transmission of BCMV in cowpea in Tamil Nadu.

Complete genome sequencing of banana bract mosaic virus infecting cardamom revealed its closeness to banana infecting isolate from India

\section{A.I. Bhat, N.S. Pamitha, A. Gopika, C.N.Biju}

Email ID for Correspondence: aib65@yahoo.co.in Division of Crop Protection, ICAR-Indian Institute of Spices Research, Kozhikode 673012, Kerala

The complete genome of the Banana bract mosaic virus (BBrMV), a Potyvirus belonging to the family Potyviridae causing chlorotic streak disease of cardamom (Elettaria cardamomum) in India was determined from a naturally infected cardamom var Njallayani Gold through reverse transcription PCR using ten sets of primers designed to different overlapping regions of the genome. The complete genome was 9712 nucleotides excluding poly (A) tail and has the genome organization that is similar to that of BBrMV isolates infecting banana and flowering ginger (Alpinia purpurata). The virus has a single open reading frame that encodes for a polypeptide which is later cleaved into ten matured proteins. The length and arrangements of different proteins in BBrMV-Cardamom was similar to other BBrMV isolates except for the $\mathrm{P} 1$ protein that showed a single amino acid deletion. Comparison with three available complete genome sequences revealed that BBrMV-Cardamom isolate is more closer to BBrMV banana isolate from India (BBrMV-TRY) than to BBrMV 
banana isolate from Philippines (BBrMV-PHI \%) and flowering ginger isolate (BBrMV-Ginger) from USA. Phylogenetic analysis also suggested that BBrMV-Cardamom isolate is closely related to other BBRMV isolates. This is first complete genome sequence of $\mathrm{BBrMV}$ isolate infecting cardamom.

Field evaluation of soybean entries under AICRP trial against yellow mosaic and bud blight disease of soybean

\author{
Ainmisha ${ }^{1}$, Vineeth Vijayan', Sanjay Kumar Lal $^{2}$ and \\ Anirban Roy ${ }^{1}$ \\ Email ID for Correspondence: ainmishasingh1@gmail.com \\ ${ }^{1}$ Advanced Centre for Plant Virology, Division of Plant Pathology, \\ Indian Agricultural Research Institute, New Delhi - 110012; \\ ${ }^{2}$ Division of Genetics, Indian Agricultural Research Institute, New \\ Delhi - 110012
}

Soybean [Glycine max (L.) Merr.] is one of the most important oilseed crops in India. Currently, it is grown in nearly 10.0mha area with average production of about $10.0 \mathrm{mt}$. However, its average productivity $(\sim 1.0 \mathrm{t} / \mathrm{ha})$ is far below the world average $(2.5 \mathrm{t} / \mathrm{ha})$. Among other factors, pest and diseases are the most important ones for such low productivity. The yellow mosaic disease (YMD) caused by whitefly transmitted begomoviruses is the most important disease of soybean. Besides YMD, recently, a thrip (Thripstabaci) transmitted tospovirus, groundnut bud necrosis virus (GBNV) appeared to be a serious concern causing bud necrosis disease (BND) in pulse crops in India. Though there is no absolute management option available against YMD and BND, but deployment of resistance is the most promising approach for their management. Breeding for resistance followed by release of new varieties through All India Coordinated Research Project (AICRP) on soybean showed promising result. In the present study we evaluated soybean entries under field condition in Delhi, a hotspot for YMV and BND. A total of 35IVT and 4 AVT entries of soybean were evaluated under epiphytotic field condition suitable for yellow mosaic and bud necrosis disease. For IVT entries two rows (5 $\mathrm{m}$ each) of individual entries were sown in non-replicated design while for AVT entries evaluation was done in replicated trials. 7 entries (PS1086, NRC124, SL1113, JS21-05, DS 3105, DS3106 and SL1104 in IVT sowed highly resistant reaction against both yellow mosaic and bud necrosis. Among the AVT entries, MACS1460 showed highly resistance against both the diseases, however, KDS869 and KDS753 though showed resistance against yellow mosaic but they are highly susceptible to bud necrosis disease. NRC753 showed highly susceptible reaction against yellow mosaic but it is highly resistant to bud necrosis. In 2017 these field resistant seven IVT and 1 AVT entries from 2016 were again grown and this year also they showed high resistant reaction under field condition.

Computational prediction of epitope-based peptides for immunodiagnosis of Chilli veinal mottle virus

\section{Akshata Gad, P. Hema Chandra Reddy and M. Krishna Reddy}

Email ID for Correspondence: akshata.gad85@gmail.com Division of Plant Pathology, ICAR-Indian Institute of Horticultural Research, Hessarghatta Lake PO, Bengaluru- 560089

Chilli veinal mottle virus (CVMV) is a flexuous filamentous particle of potyvirus genus, is endemic virus in hot pepper mainly in Asian countries. This virus limits the chilli cultivation in India and diagnosis is often confused with CMV, TMV and PMMV. For quick diagnosis antibody based detection is good for large sample size. For the effective diagnosis, designing antigenic peptides based on coat protein using computational methods. The Predicted MHC class I and class II binding peptides of the coat protein which can be dominant element in serodiagnosis, may be adequate to evoke immune response and target virus detection. 15 numbers of peptides with high score were predicted toward the TAP transporter, "SEKPYMPRY" ranked 8.220 as the highest score. The coat protein 3D structure was modelled with the template "5ODV" of PDB ID. The template being the structure of watermelon mosaic potyvirus, has 100 confidence value and $66 \%$ of identity to the input sequence. Besides this analysed the protein residual properties along with the structure, where total number of negatively (Asp + Glu) and positively (Arg + Lys) charged residues were found to be 38 and 36 respectively. Further analysis found that the protein is highly built with the alpha helix (43.55\%). MHC Class II epitope identification and selection resulted in 279 numbers of nonamers from input sequence. The peptide binders of MHC class II chosen from MHC II-IAb, MHC II-IAd, MHC II-IAg7 and MHC II-RT1.B derived from SVM based classifier predicted "RHTAEDVNR", "GVSNRMFGL", "HIQMKAAAL", "TERHTAEDV" peptides respectively. Molecular Docking of proteinepitope using AutoDock Vina predicted binding of epitopes to their respective molecules.

\section{Identification of tomato leaf curl Gujarat virus infecting Xanthium strumarium a new host for begmoviruses}

\section{K.V. Ashwathappa, P. Swarnalatha, S. Jalai and M. Krishna Reddy}

Email ID for Correspondence: mkreddy@iihr.res.in Division of Plant Pathology, ICAR-Indian Institute of Horticultural Research, Hessaraghatta Lake PO, Bengaluru-560089

During the survey of viruses on vegetable crops and associated weeds in different parts of Bangalore rural, yellowing, upward leaf curling and crinkling symptoms were recorded on Xanthium strumarium plants. The incidence of such symptoms varied from 7.5 to $51.7 \%$ in different locations. Total DNA was extracted by CTAB method and by using begomovirus specific primers full length genome of DNA-A and associated beta satellite were amplified through PCR. The same samples were also amplified for DNA-B using ToLCNDV DNA B specific primers. PCR analysis of 12 plant samples was done, among which 10 showed amplification of expected size. The amplified product was cloned and fully sequenced. Sequence analysis in NCBI blast research showed sequence identity of $97-98.5 \%$ with Tomato leaf curl Gujarat virus. The DNA-B genome sequences has shown nucleotide identity of 87. 9-91.5\% with ToLCNDV DNA-B genome. The use of specific primers for DNA-A and DNA-B in PCR resulted in amplification of a full length $\sim 2.7 \mathrm{~kb}$ product, confirming the bipartite nature of virus. This was supported by close clustering of begomovirus of the current study with the Tomato leaf curl Gujarat virus by phylogenetic analysis. This is the first report of occurrence of Tomato leaf curl Gujarat virus on $X$. strumarium, a common weed from India.

Molecular characterization of begomoviruses from ornamental plants in Tamil Nadu

\section{S. Deepan, R. Pandiyan, D. Michael Immanuel Jesse, T. Kumaran, S.U. Mohammed Riyaz and K. Kathiravan}

Email ID for Correspondence: deepanvit@gmail.com Plant Molecular Virology and Nanobiotechnology Laboratory, Department of Biotechnology, University of Madras, Maraimalai Campus, Guindy, Chennai- 600025 
Begomovirus is one of the largest genus of the family Geminiviridae transmitted by vector whitefly (Bemisia tabaci). In recent years, gemini virus infection is randomly spreading to major crops, weeds and also in ornamental crops. The present study we identified a begomovirus infecting major crops and ornamental plants cultivated in some parts of Tamil Nadu. Leaf Curl disease in plants are emerged as serious problem in Tamil Nadu. During 2014, very high disease incidence (up to $90 \%$ of plants) was observed in major crops like Lycopersicum solanum, Benincasa hispida and ornamental plants like Jasmine, (Jasminum) and Crossandra infundibuliformis. The characteristics field symptoms were upward severe curling and reduced size of leaves. Severely affected plants were stunted and produced small and no flowers. The presence of Begomovirus was confirmed by polymerase chain reactions (PCR) using the coat protein and Replicase gene primer designed by us using Bioedit Version software, which gave an approximately $500 \mathrm{bp}$ and $850 \mathrm{bp}$ amplicon were obtained the same were sequenced. A BLAST search of GenBank revealed close (96\%) similarity of the sequence with Tomato leaf curl New Delhi virus from the NCBI database. Hence the virus isolated from ornamental plants like Crossandra infundibuliformis which may act as a host reservoir for Begomoviruses in Tamil Nadu.

\section{Intracellular trafficking and targeting of carmovirus movement} protein Dyads (7A and 7B) to plasmodesmata

\section{N. Dileep Kumar, Sourav pal, Nunna Haritha and Gopinath Kodetham}

Email ID for Correspondence: dileepmscbiotech@gmail.com Department of Plant sciences, School of life science, University of Hyderabad, Hyderabad-500046, Telangana, India

Plant virus infection depends on the proper targeting and interaction or insertion of the movement proteins (MPs) with or into the endoplasmic reticulum (ER). Cell-to-cell transport of plant virus(es) requires the virally encoded MPs. These proteins specialize in the translocation of the viral genome(s) or in some cases, the virions from the replication/encapsidation site to adjacent cells through the plasmodesmata (PD). Often, the cell-to-cell transport of plant viruses depends on one or more virally-encoded MPs and in few cases coat protein may be required as an axillary protein to target and to dilate the PD. Some MPs are integral membrane proteins that interact with the membrane of the endoplasmic reticulum, however, a detailed understanding of the interaction between MPs and biological membranes, the available literature is scanty. Towards this, we have designed experiments to elucidate the intracellular trafficking and targeting of MP to the plasmodesmata in the whole plant scenario. In our laboratory, we are using a newly isolated Carmovirus member from melon plants as a model virus and designated as Melon necrotic spot virus (MNSV-HYD) for parity. MNSV-HYD particles are spherical in shape and encapsulate a $4.3 \mathrm{~kb}$ positive sense single stranded RNA molecule as its genetic material. The genome contains five open reading frames, encoding 28, 89, 7(A), 7(B) and $42 \mathrm{kDa}$ proteins from $5^{\prime}$ to $3^{\prime}$ orientation respectively. Centrally located two ORF's separated by a stop codon encode two small proteins of $7 \mathrm{kDa}$ and are designated as 7A and 7B dyad forms the functional movement protein complex. Using in silico analysis, we have predicted a transmembrane domain (TMD) in 7B protein with 19 amino acid length with single hydrophobic amino acid stretch at N-terminus. We presume that TMD is playing pivotal role in membrane integration of 7B protein to plasmodesmata. To unravel the mechanism 7A and 7B proteins to form a functional MP complex and to understand intracellular trafficking and targeting to the PD, several 7A and 7B-GFP/ Cherry chimeras were constructed and cloned into pCB302 binary vector and electrophoretically mobilized into Agrobacterium EHA105 strain. Agro-infiltration of individual and combination(s) of chimeras are infiltrated into Nicotiana bethamiana and transient expressions are extensively analysed by confocal laser scanning microscopy and the results are documented will be discussed at virocon 2017.

Assessment of efficiency of DNA virus based VIGS system for silencing the bacterial leaf blight disease resistance gene Xa38 in rice

\section{Kamlesh Kumari ${ }^{1}$, Ravi Kant ${ }^{2}$, Prashant Mishra ${ }^{3}$, Rhitu Rai ${ }^{3}$, Indranil Dasgupta ${ }^{1}$}

Email ID for Correspondence: kamlesh.kumari407@gmail.com ${ }^{1}$ Department of plant molecular biology, University of Delhi South Campus, New Delhi, India-110021; ${ }^{2}$ Plant Microbe Interaction Lab, National Institute of Plant Genome Research, New Delhi, India110067; ${ }^{3}$ ICAR-National Research Center on Plant Biotechnology, Pusa Campus, New Delhi-110012

Virus induced gene silencing (VIGS) is a reverse genetics tool, which exploits plant innate defense mechanism, post-transcriptional gene silencing (PTGS) to specifically silence endogenous genes utilizing recombinant viruses. VIGS has become an extensive and indispensable tool for the functional analysis of plant genes in post-genomics era. Many VIGS systems have been developed and widely adapted for dicots so far; however, developments of monocots-specific VIGS tools are relatively lesser. Our lab developed Rice tungro bacilliform virus (RTBV) DNA based VIGS system for functional analysis of genes in rice. Present study aims to utilize RTBV-VIGS system to identify the candidate gene responsible for bacterial blight resistance in Xa38 loci against the causal pathogen Xanthomonas oryzae pv. oryzae. Xa38 is a dominant resistance gene identified in Oryza nivara IRGC81825 and introgressed into susceptible cultivated variety PR114. Xa38 [previously designated as $\mathrm{Xa3O}(t)$ ], has been mapped to a $38.4 \mathrm{~kb}$ region on the long arm of rice chromosome 4 . We targeted LOC_Os04g53030, out of the three loci, LOC_Os04g53030, LOC_Os04g53050 and LOC_Os04g53060, proposed as putative candidate genes for Xa38 mediated resistance. Using ORF finder, two longest ORFs (ORF I and ORF III) were selected for VIGS mediated gene silencing. Agrobacterium mediated inoculation of recombinant VIGS-construct in PR114 (Xa38) resulted in 50-60\% reduction of Xa38 transcript accumulation as compared to control plants using Real-time PCR. We further subjected the plants to leaf clip infection assays with virulent Xoo strain to assess the effect of silencing ORFI and ORFIII of the target gene. Results revealed enhanced susceptibility in the silenced plants as compared to non-silenced plants. The present study provides the molecular and functional evidence for pRTBV-VIGS as an efficient system for functional genomics in rice, successfully utilized here to identify the resistance gene in Xa38 loci.

Infectivity analysis of Okra (Abelmoschus esculentus) associated geminiviruses and betasatellite by agroinoculation in Okra and Nicotiana benthamiana

\section{Kanika Gupta, Rashmi Rishishwar, Indranil Dasgupta}

Email ID for Correspondence: kanikagupta1903@gmail.com Department of Plant Molecular Biology, University of Delhi South Campus, New Delhi, India-110021

Okra (Abelmoschus esculentus) is an important vegetable crop in India. The Yellow Vein Mosaic disease (YVMD) of okra is caused by a complex consisting of the monopartite begomovirus Bhendi yellow vein mosaic virus (BYVMV, family: Geminiviridae) and a 
betasatellite component, Bhendi yellow vein mosaic betasatellite (BYVMB). Infectivity analysis is important to study the aetiology of the BYVMD, resistance screening, host range and to alleviate the crop losses due to okra related geminiviruses. We have earlier reported the association of Mesta Yellow Vein Mosaic Virus (MeYVMV) with YVMD. Here we report the results of our study to test the infectivity of cloned BYVMV, MeYVMV and BYVMB, isolated from field-infected okra plants in okra and the laboratory plant Nicotiana benthamiana. Using agroinoculation, we observed accumulation of viral DNA in inoculated $N$. benthamiana plants by 21 st day post-inoculation (dpi) without any symptom development only in certain combinations of the viral and satellite DNAs. In Okra, we observed PCR-based amplifications of the viral DNA in expanded cotyledons and leaves at $20 \mathrm{dpi}$ in experiments where germinating Okra seeds were inoculated. This was observed only when both viral and betasatellite DNAs were used as inocula. The results suggest that the cloned viral and betasatellite DNAs, although showing accumulation in the inoculated plants are able to cause cryptic infections without causing symptoms. The procedure is being standardized to obtain a rapid screening procedure for resistance against BYVMD in Okra.

\section{Adroit role of $26 \mathrm{~S}$ proteasome subunit RPT4a in tomato for regulation of ToLCNDV}

\section{Pranav Pankaj Sahu, Namisha Sharma, Swati Puranik, Supriya Chakraborty and Manoj Prasad}

Email ID for Correspondence: namisharma.ns@gmail.com National Institute of Plant Genome Research, New Delhi-110067

Ubiquitin/26S proteasome (UPS) pathway has been implicated in diverse aspects of eukaryotic cell regulation as it rapidly removes intracellular proteins. In addition to these functions, involvement of $26 \mathrm{~S}$ proteasomal subunits in plant pathogen-interactions, and the roles of each subunit in independently modulating the activity of many intra- and inter-cellular regulators controlling physiological and defense responses of a plant were well reported. In this regard, we aimed to functionally characterize a Solanum lycopersicum 26S proteasomal subunit RPT4a (SIRPT4) gene, which was differentially expressed after Tomato leaf curl New Delhi virus (ToLCNDV) infection in tolerant cultivar H-88-78-1. Molecular analysis revealed that SIRPT4 protein has an active ATPase activity. SIRPT4 could specifically bind to the stem-loop structure of intergenic region (IR), present in both DNA-A and DNA-B molecule of the bipartite viral genome. Lack of secondary structure in replication associated gene fragment prevented formation of DNA-protein complex suggesting that binding of SIRPT4 with DNA is secondary structure specific. Interestingly, binding of SIRPT4 to IR inhibited the function of RNA Pol-II and subsequently reduced the bi-directional transcription of ToLCNDV genome. Virus-induced gene silencing of SIRPT4 gene incited conversion of tolerant attributes of cultivar H-88-78-1 into susceptibility. Furthermore, transient over-expression of SIRPT4 resulted in activation of programmed cell death and antioxidant enzymes system. Overall, present study highlights non-proteolytic function of SIRPT4 and their participation in defense pathway against virus infection in tomato.
Molecular detection of bean common mosaic necrosis virus in Dolicus bean (Lablab purpureus)

\section{B. S. Pavithra, M. Krishna Reddy, Salil Jalali and D.K. Samuel}

Email ID for Correspondence: pavithrabs24@gmail.com) Division of Plant Pathology, Indian Institute of Horticultural Research,, Hessarghatta Lake P.O., Bengaluru -560089, India

Bean common mosaic necrosis virus (BCMNV; Family Potyviridae, genus Potyvirus) infects legume crops in many regions of the world. It is transmitted by aphids in non-persistent manner and by seed. Dolicus bean (Lablab purpureus L.) is an important legume crop widely cultivated in India. The bean plants showing mosaic mottling leaf deformation, necrosis and malformation of leaves were collected from Meghalaya. DAC-ELISA was performed by using a universal Potyvirus antiserum on symptomatic leaf samples collected from different locations of Meghalaya. All the samples showed positive reaction to potyvirus antisera. Electron microscopic observation of sap extracted from infected leaves revealed the presence of flexuous filamentous shaped particles of about $750 \mathrm{~nm}$ long. To further characterize the virus, the infected leaves were mechanically inoculated on Phaseolus vulgaris, Vigna unguiculata, Glycine max, Lablab purpureus and Chenopodium amaranticolor. Phaseolus vulgari, Glycine $\max$ and $V$. unguiculata plants showed mosaic symptoms followed by systemic necrosis. Chenopodium amaranticolor showed chlorotic local lesions on inoculated leaves. Total RNA was isolated from infected plants using Trizol Reagent, Reverse transcription (RT)-PCR was carried from the RNA using potyvirus group specific primers amplified $1.76 \mathrm{~kb}$ from virus infected sample but not from healthy control plants. Cloning and sequence analysis showed maximum of $90 \%$ amino acid and $94 \%$ of nucleotide sequence identity in the CP region and $95 \%$ nucleotide homology in the $3^{\prime}$-UTR with the NL8 strain of BCMNV. Based on electron microscopy and sequence analysis the virus infecting Dolicus bean has been identified as BCMNV and to our knowledge this is the first report of occurrence of $\mathrm{BCMNV}$ in India.

\section{Molecular characterization and recombination analysis of Cotton leaf curl Multanvirus: a whitefly transmitted geminivirus} in Northern India

\section{Razia Qadir, Jawaid A. Khan}

Email ID for Correspondence: raziaqadirwathloo@gmail.com Plant Virus Laboratory, Department of Biosciences, Jamia Millia Islamia, Jamia Nagar, New Delhi -110025

Cotton leaf curl disease (CLCuD) is an imminent threat to cotton production in India and other cotton producing countries. CLCuD is caused by a complex of begomoviruses of the family Geminivirideae in association with satellite molecules and is transmitted by the whitefly vector Bemisia tabaci. In this study begomovirus affected leaves of cotton plants were collected from Haryana and New Delhi. Rolling circle amplification was performed for detection and characterization of viruses. Cotton leaf curl virus $(\mathrm{CLCuV})$ genome and its associated satellite molecules were amplified, cloned and sequenced. Sequence analysis showed that the begomovirus genomes shared highest (95-96\%) identity with previously reported Cotton leaf curl Multan virus (CLCuMuV). Betasatellite molecules associated with these begomoviral genomes shared highest identity (95-99\%) with Cotton leaf curl Multan Betasatellite (CLCuMB) from India and alphasatellite molecules shared highest identity (98\%) with Guar leaf curl alphasatellite (GLCuA) previously reported from Pakistan. It is concluded that CLCuMuV is s stable and dominant population in 
Northern India, spreading at an alarming rate which can be a potential threat. Further Phylogenetic and recombination analysis suggested that these viruses evolve via recombination between CLCuMuV and Cotton leaf curl Kokhran virus (CLCuKV). Our results revealed that there are the potential sites of recombination in the begomovirus genomeand may lead to sequence variability in these strains. Further, suggesting that new variants of begomovirus are rapidly spreading and causing leaf curl disease in cotton in Northern India.

\section{Role of ubiquitin-proteasomal pathway genes in plant-virus interaction}

\section{Ritika Kulshreshtha, Namisha Sharma, Manoj Prasad}

Email ID for Correspondence: ritushina@gmail.com

National Institute of Plant Genome Research, New Delhi, 110067

Tomato leaf curl New Delhi virus (ToLCNDV) has emerged as a serious problem in the production and propagation of tomatoes. Around $90 \%$ of yield loss has been reported in India. Hence there is an immediate need to implement an effective strategy, which could prevent from yield loss caused by this virus. Various efforts has been implicated by the virologists to generate virus resistance/tolerant in tomato. Various genes have been identified in this context. For example, a suppressive subtractive hybridization ( $\mathrm{SSH}$ ) approach identified 106 differentially expressed genes in a tolerant cultivar of tomato, namely cv. H-88-78-1 in which SlyUCE2 was found to be differentially expression. In this study, we aimed to functionally characterize these genes through transgenic and virus induced gene silencing (VIGS) approach. We silenced the SlyUCE2 with the help of the VIGS technique which transformed the tolerant cv. H-88-78-1 into susceptible plant. Southern hybridization showed an increase in the accumulation of viral particles in SlyUCE2-silenced plants. To unravel the downstream mechanism, interaction of SlyUCE2 was checked with other ubiquitin-proteasomal pathway genes, enlisted in $\mathrm{SSH}$, through Yeast-two hybridization, and interestingly ARM, a ubiquitin-ligase was found to be interacting with it. In-silico analysis of SlyUCE2 identified few probable interacting proteins, broadly categorized as: Ubiquitin conjugating enzyme, Ubiquitin activating enzyme and Ubiquitin ligase. In transgenic approach, SlyUCE2 was over-expressed in Pusa Ruby, a susceptible cultivar against ToLCNDV through Agrobacterium mediated transformation. This study provides a novel finding that ubiquitin proteosomal pathway genes are actively involved in providing defense against ToLCNDV.

Deciphering the role of stress responsive RNA helicases in tomato against biotic and abiotic stresses

\section{Saurabh Pandey, Vaishali Chaudhry, Shweta Shweta, Manoj Prasad}

Email ID for Correspondence: pandey.saurabh784@gmail.com National Institute of Plant Genome Research, New Delhi

Plant stresses including biotic and abiotic stresses adversely affect plant growth and limit agricultural production worldwide. Minimizing these losses is the major area of concern for world food security. Therefore, it is necessary to develop multiple stress tolerant cultivars. RNA helicases are highly conserved plant proteins contributing in all form of RNA metabolism. Although RNA helicase association with a range of cellular functions is well recognized, their prominence in response to abiotic and biotic stress is merely beginning to emerge. Hereof an attempt to identify and characterize RNA helicases in tomato plant has been made. In this study, in silico analysis revealed a total of 131 RNA helicase genes in tomato genome. According to the structural features of the motif II region, we divided the tomato RNA helicase genes into DEAD-box (42), DEAH-box (38) and DExD/Hbox (51) helicase genes. However, there were 29 RNA helicases which did not belonged to either of three subfamilies, and further grouped into "other helicase". We also aimed to study the role of DEAD-box helicase in the tomato against the Tomato leaf curl New Delhi virus (ToLCNDV). Based on our previous study, we selected 3 putative candidate DEAD-box helicase genes, belonging to same clade. Their differential expression pattern was confirmed with the real time PCR. Out of three genes, DEAD-box helicase SIDBP-12 showed upregulation in the tolerant cultivar H-88-78-1 during ToLCNDV infection and further characterized through Virus Induced Gene Silencing (VIGS) which signified its function in viral tolerance. Moreover, expression of selected genes shows differential expression of RNA helicases during different abiotic stresses. Further, heterologous overexpression of $S I D B P-12$ in yeast conferred tolerance to heat, salt and cold stresses. Our study indicate $S I D B P-12$ as a potential candidate for providing biotic and abiotic stress tolerance, and details of precise mechanism is under progress.

\section{Designing and development of a novel RNAi gene construct against Cotton leaf curl Multan virus}

\section{Shahfahad Alam, Jawaid A. Khan}

Email ID for Correspondence: ansari.fahad009@gmail.com Plant Virology Laboratory, Department of Biosciences, Jamia Millia Islamia (A Central University), New Delhi-110025, India

Cotton (Gossypium hirsutum), belonging to the family Malvaceae, is one of the important economical crop of India. India is the largest producer of cotton in the word. Cotton leaf curl disease (CLCuD) is the major factor for the loss of cotton cultivation. CLCuD is caused by $\mathrm{CLCuV}$ species in association with satellites viz. alphasatellite and betasatellite. $\mathrm{CLCuV}$ belongs to the genus Begomovirus (family Geminiviridae) and transmitted by whitefly (Bemisia tabaci). Cotton plants showing typical leaf curling, vein thickening and enation on the abaxial side of leaf were collected from Haryana, India. Total genomic DNA was isolated from diseased cotton leaves and subjected to rolling circle amplification for begomovirus genome amplification. A silencing suppressor gene of the begomovirus was amplified, cloned and sequenced. Initially PCR amplified $\operatorname{TrAP}$ (transcriptional activator protein) gene was cloned into TA-cloning vector in an antisense orientation and then intron was cloned in this vector. Sensed oriented $\operatorname{TrAP}$ was amplified and cloned. The whole RNAi cassette of silencing suppressor gene was developed, confirmed by restriction digestion and sequencing. The complete RNAi construct was cloned into binary vector, mobilized into Agrobacterium tumefaciens. Gossypium hirsutum was transformed using RNAi construct through Agrobacterium-mediated transformation using embryo apex explants. Further experiments to know the effect and potential RNAi construct against $\mathrm{CLCuV}$ infection are in progress.

Chickpea stunt: a complex disease caused by Chickpea chlorotic dwarf virus and candidatus phytoplama trifoli in India

\section{Y. S. Shreenath, R.K. Saritha, Rajendra Kumar Pant and G.P. Rao}

Email ID for Correspondence: gprao_gor@rediffmail.com Division of Plant Pathology, Indian Agricultural Research Institute, Pusa Campus New Delhi 110012, India 
Suspected phytoplasma and virus-like symptoms which includes pale green leaves, bushy appearance due to excessive stunting of shoots, reduced internodal length and excessive axillary shoot proliferation were recorded on chickpea at experimental fields of Indian Agricultural Research Institute, New Delhi, India in February-March 2017. The association of phytoplasma was confirmed in symptomatic chickpea plants by direct and nested PCR assays with phytoplasmaspecific universal primer pairs (P1/P6 and R16F2n/R16R2n). Analysis of the sequences revealed that $16 \mathrm{Sr}$ DNA sequence of chickpea stunt phytoplasma isolate had $99 \%$ sequence homology with strains of 16SrVI group phytoplasmas. The Chickpea chlorotic dwarf virus (CpCDV) (genus Mastrevirus, family Geminiviridae) coat-proteinspecific primers: MCPF (5' GCAGGTGGCTACCTTTTC $3^{\prime} / \mathrm{MCPR}$ $\left(5^{\prime}\right.$ ATTGCCAACGGACTTGAAG $3^{\prime}$ ) yielded an amplicon of approximately $596 \mathrm{bp}$ from the same symptomatic chickpea stunt samples confirming the presence of $\mathrm{CpCDV}$. The coat protein sequence of the CpCDV New Delhi isolate revealed 99\% sequence identity with the CP sequences of Chickpea chlorotic dwarf Pakistan virus. Our study suggested a mixed infection of Candidatus Phytoplama trifoli and mastrevirus associated with chickpea stunt disease. This is first report of mixed infection of virus and phytoplasma associated with chickpea stunt disease from the world.

\section{Identification and molecular diagnosis of viruses infecting cucumber}

\section{Suveditha S., P. Swarnalatha and M. Krishna Reddy}

Email ID for Correspondence: suvi.ss519@gmail.com Division of Plant Pathology, ICAR- Indian Institute of Horticultural Research, Hessarghatta Lake PO, Bengaluru -89

Cucumber is one of the important vegetable crop grown throughout the country and virus diseases of late became major limiting factor in its cultivation and low productivity both in open field and protected cultivation. Survey conducted in the vegetable growing regions of Andhra Pradesh and Karnataka indicated incidence of virus and virus like diseases ranging from 15.6 to $85.0 \%$ in different locations. Symptomatic samples of cucumber leaves showing the symptoms of chlorotic lesions, yellowing, Interveinal chlorosis, leaf crinkling, curling, vein twisting, thickening of older leaves and necrotic lesions were collected and were tested for the presence of virus by mechanical transmission, electron microscopy, PCR and RT-PCR. Virus infected leaf samples washed with RNase-free sterile double distilled water and sap extracted when examined under Electron microscope, showed the presence of isometric particles. Further to identify the causal virus, total RNA was isolated from symptomatic and healthy leaf samples of cucumber using tri-reagent and was used for RT-PCR amplification using Polerovirus specific primers and coat protein specific TSV primer. RT-PCR amplification resulted in amplification of a single DNA fragment of approximately $0.3 \mathrm{~kb}$ specific to Polerovirus and $0.8 \mathrm{~kb}$ specific to TSV, from symptomatic samples but not from healthy control and asymptomatic samples. Cloning, sequencing and nucleotide blast search in NCBI showed more than $93 \%$ with Cucurbit- aphid borne yellow virus (Genus: poleroviruses; Family: Luteoviridae) and $92-95 \%$ similarity with already reported TSV (Genus: Ilaravirus; Family Bromoviridae). The DNA isolated form infected virus symptomatic and healthy samples were used for PCR amplification using begomovirus specific primers, amplified DNA fragments of 0.7, 1.2 and $1.4 \mathrm{~kb}$ from infected samples but not from healthy control. Cloning, sequencing and analysis of DNA fragments indicated 92-98\% similarity with ToLCNDV (Genus: Begomovirus; Family Geminiviridae).

\section{Characterization and biopassay Spilosoma obliqua} nucleopolyhedrovirus

\section{Tanuja $\mathbf{S}^{1}$, Sivakumar $\mathbf{G}^{2}$, Muthuraju $\mathbf{R}^{1}$, Surabhi Kumari ${ }^{2}$, Raveendran $\mathbf{P}^{2}$}

Email ID for Correspondence: tanuja5112@gmail.com ${ }^{1}$ College of Agriculture, GKVK, UAS, Bengaluru, Karnataka, India, 560 065; ${ }^{2}$ National Bureau of Agriculturally Important Insects, Bengaluru, Karnataka, India, 560024

Spilosoma (Spilarctia) oblique (Walker) (Arctiidae: Lepidoptera), is a sporadic and polyphagous insect pest widely distributed throughout India. It is reported to attack 126 plant species belonging to 25 families, including pulses, oilseeds, cereals, vegetables, mulberry, turmeric, medicinal and aromatic plants, causing heavy economic loss. The caterpillars feed gregariously during the early larval stages and solitarily in the late larval stages. Chemical control of this pest is difficult and uneconomical because the pest also feeds on several weed plants. Chemicalisation is no longer considered sustainable since several agrochemicals when used indiscriminately has led to resistance development besides causing serious environmental problems. Consumer concern about chemical pesticide residues on food is driving the search for an alternative method of pest control. New research suggests that bio-control technology, using insect viruses, could be an effective alternative, which is environmental friendly and excellent alternative for management of S.obliqua. During survey in and Bengaluru, India, an epizootic like condition was observed in the field population of $S$. obliqua caused by a nucleo polyhedrovirus (NPV). The virus was extracted from the diseased larvae collected from the field and was purified for further works. To determine the identity, scanning electron microscopy revealed that the occlusion bodies (OBs) of Spilosoma oblique NPV (SpobNPV) appeared tetrahedral in shape and the size of the polyhedra ranged from 1.2 to $2.0 \mu \mathrm{m}$, besides some OBs were irregular in shape. The LC50 value of SpobNPV against second instar larvae was $3.2 \times 10^{4} \mathrm{OBs} / \mathrm{ml}$ for by leaf disc method.

\section{Antisense RNA: mediated resistance against leaf curl disease in papaya plant}

\section{Vartika Sinha $^{1}$, N.B. Sarin ${ }^{2}$, D.Bhatnagar ${ }^{3}$}

Email ID for Correspondence: vartika.sinha2009@gmail.com ${ }^{1}$ Department of Genetics, University of Delhi South Campus, Benito Juarez Marg, New Delhi-110021; ${ }^{2}$ School of Life Sciences, Jawaharlal Nehru University, New Mehrauli Road, New Delhi110067, India; ${ }^{3}$ School of Biochemistry, Devi Ahilya University, Indore-452017, India

Geminiviral diseases, particularly Papaya Leaf Curl Disease caused by begomovirus, is the most important constraint to the production of papaya in the tropical and subtropical regions of the world. Begomoviruses have increased pathogenicity because of their adaptation to a wide host range; consequently, these viruses cause a major loss to agro-economic crops worldwide. To overcome this problem, various non-conventional and conventional control measures based on the chemical control of whiteflies and conferring genetic resistance or tolerance against the virus, respectively, have been applied for decades. Moreover, pathogen derived resistance PDR-mediated approaches have been introduced, in which plant resistance to the infecting virus is mediated either by the expression of a particular gene or by target mRNA degradation inside the host cell. Among PDR-mediated approaches, RNAi technology is currently widely used for crop improvement. RNA silencing is a homology-dependent 
gene silencing mechanism that is guided by small RNAs (18-25 nucleotides long) and mediated by siRNAs and miRNAs. In this study, we designed a gene construct representing an antisense coat protein gene. We also analyzed the efficacy of the induced resistance against Croton yellow vein mosaic virus (CrYVMV) affecting papaya (found in this study) in Nicotiana tabacum plants. Control plants developed typical leaf curl symptoms, whereas transgenic plants were symptomless. Moreover, the key component (i.e., short interfering RNA) of the antisense pathway was upregulated in transgenic plants. This finding demonstrates the activation of the gene silencing mechanism in transgenic plants. Thus, these results confirm that our construct is functional and effectively induces transient resistance against CrYVMV infections.

Evaluation of biocides and phyto: chemicals for the management of thrips and groundnut bud necrosis virus (GBNV) disease in groundnut

\section{Vijayalakshmi G' ${ }^{1}$, N. Ganapathy ${ }^{1}$, G. Karthikeyan ${ }^{2}$ \\ Email ID for Correspondence: vijaya.vijitha18@gmail.com ${ }^{1}$ Department of Agricultural Entomology, Tamil Nadu Agricultural University, Coimbatore - 641003, India; ${ }^{2}$ Department of Plant Pathology, Tamil Nadu Agricultural University, Coimbatore - 641003, India}

The Groundnut bud necrosis virus (GBNV) and its propagative vector thrips are a menance in groundnut cultivation in India. A study was undertaken for the management of GBNV and its vector using biocides and phyto-chemicals. The field experiments conducted using the biocides and phyto-chemicals viz., sorghum leaf extract (10\%), coconut leaf extract (10\%), quinalphos $(0.05 \%) 25$ EC, combination with quinalphos $25 \mathrm{EC}(0.05 \%)$, yellow sticky trap, yellow sticky trap combined with ethyl nicotinate $1500 \mathrm{ppm}$ impregnated pheromone trap, Pseudomonas fluorescens and fermented buttermilk in locations revealed that sprays of sorghum leaf extract $(10 \%)+$ quinalphos $25 \mathrm{EC}(0.05 \%)$ and coconut leaf extract $(10 \%)+$ quinalphos $25 \mathrm{EC}(0.05 \%)$ were significantly effective in reducing the thrips population and incidence of GBNV and increasing the yield of groundnut when compared to control. Among the treatments, leaf extracts combined with quinalphos resulted in reducing the thrips population to an extent of 66 per cent and GBNV incidence of 6 per cent when compared to control (27.7 per cent of incidence).

\section{Animal Virology}

\section{Oral Presentation}

Molecular characterization of complete genomic segment-2 (RdRP encoding gene) of picobirnaviruses detected in different animal host species

\section{Souvik Ghosh}

Email ID for Correspondence: souvikrota@gmail.com; sghosh@rossu.edu Department of Biomedical Sciences, Ross University School of Veterinary Medicine, Saint Kitts and Nevis, West Indies

Picobirnaviruses (PBVs), family Picobirnaviridae, are opportunistic enteric pathogens that infect a wide variety of mammals and avian species. The PBV genome consists of bi-segmented double-stranded
RNA. The PBV gene segment-1 ( 2.2-2.7 kb) codes for the viral capsid protein and a putative polypeptide of unknown function, whilst gene segment-2 $(\sim 1.2-1.9 \mathrm{~kb})$ encodes for the viral RNA-dependent RNA polymerase (RdRp). Until now, most studies on PBV genomics were based on partial gene segment- 2 sequences ( $\sim 200 \mathrm{bp})$, information on which is not sufficient to study the features of putative RdRps, or genetic makeup of PBVs. During the last decade, we reported PBVs from different animal species, expanding the host range of these viruses. Using a non-specific primer-based amplification method with modifications, we could successfully determine the full-length nucleotide (nt) sequences of gene segment-2 of PBV strains detected in a cow, a cat, a dog and captive green/vervet monkeys. The gene segment- 2 of the bovine, canine, feline, and simian PBV strain was 1758 bp, 1689 bp, 1784 bp, and 1707 bp long, respectively, and encoded a putative RdRp of 554 amino acid (aa), 531 aa, 534 aa, and 538 aa, respectively. By nt and deduced aa sequence identities and phylogenetic analyses, the complete gene segment-2 of the bovine, canine, feline and simian PBV strains exhibited a high degree of genetic diversity with those of PBVs from other host species. On the other hand, these PBV strains were found to retain the $5^{\prime}$ - and $3^{\prime}$ - end nt sequences and the three domains of putative RdRp that are conserved in PBVs. This is the first report on molecular characterization of complete gene segment- 2 of PBV strains detected in cattle, cats, dogs and green/vervet monkeys, allowing us to study the features of putative RdRps, and genetic makeup and evolution of PBVs in these host species.

\section{Viral zoonoses and the role of animal-human interface on one} health

\section{Nagendra R. Hegde}

Email ID for Correspondence: hegde@niab.org.in National Institute of Animal Biotechnology, Hyderabad

Life forms on earth exist in a delicate balance, which has all too often been disturbed by natural and anthropogenic causes. Besides natural cycles such as arthropod-borne dissemination, domestication of animals, human-animal conflict, and habitat intrusion have led to transmission of infectious agents, including viruses, from animals to humans. The interdependent coexistence of humans with production and companion animals, and habitat overlap with wild animals frequently impacts public health. Indeed, a large majority of emerging and reemerging diseases are zoonotic, including West Nile fever, SARS, MERS, Ebola hemorrhagic fever, influenza etc., are zoonotic viral diseases. Control of such diseases encompasses understanding their biology, identification of reservoir animals and methods of transmission, and development of rapid diagnostic tests and therapeutic and prophylactic strategies. At the same time biodefense, biosecurity, containment and other scientific and ethical issues need further discussions. Most importantly, there is a need for seamless multidisciplinary approach which includes epidemiologists, modelers, economists, sociologists, ecologists and behavioral biologists, environmental researchers, basic and translational biology researchers (both academic and industry), clinicians (both medical and veterinary), community leaders and governments in order to attack viral (and other) zoonoses. Although these topics have been reiterated earlier, it is time for us to initiate concrete steps to invest in multidisciplinary programs addressing some of the most important zoonotic diseases in India and globally. 
History and current trends in bovine rotavirus research in India

\section{Yashpal S. Malik}

Email ID for Correspondence: malikyps@gmail.com Indian Veterinary Research Institute, Izatnagar 243122, Uttar Pradesh, India

In recent times, the virus induced enteritis has acclaimed a major disquiet throughout the world. Since its first record in 1969, rotavirus is recognized as the foremostsource of diarrhoeal morbidity and mortality worldwide affecting mammals and birds. Prevention of rotavirus-associated disease is a global public health goal and simply adherence to good hygiene and sanitation wouldn't be sufficient enough to prevent occurrence and spread of rotavirus infection. For the control of the virus infection regular monitoring of newly emerging rotavirus is needed. Although considerable research has been carried out on rotavirus disease in humans in India, a comprehensive data on the patterns of distribution of rotavirus genotypes ( $\mathrm{G}$ and $\mathrm{P}$ types) in animal species is still lacking. Being a segmented genome virus, rotavirus evolves very rapidly through a number of mechanisms and creates pressure on epidemiological surveys. Genetic drift and genetic shift are the two common ways by which rotaviruses evolve in nature. The available literature reveals marked diversity among rotavirus strains circulating in India with emergence of certain unusual strains. The epidemiological statistics generated through $\mathrm{G}$ and $\mathrm{P}$ genotyping of bovine rotaviruses in various epidemiologic surveys confirms that G3, G6, G8 and G10 are the more common G genotypes circulatingin the country; unusual G types, such as G15 with $\mathrm{P}[15]$ and $\mathrm{P}[21]$ types are localized in some parts of the country, and multiple $G$ and $P$ types can co-circulate within the same region and can cross the inter-geographical boundaries of the states. Among all the rotaviruses, group A and B viruses have been recognized in bovine population, of which group $\mathrm{A}$ is the most significant cause of neonatal bovine diarrhoea. Of the note, change in the pattern of predominance of bovine rotavirus genotypes has been observedduring the last one decade. As since 2003, G3 genotype has shown an increasing trend in many parts of India. Few of the bovine rotavirus strains also exhibited unique genomic constellations with evidence of interspecies transmission, even involving human-animal reassortants. The high prevalence suggests that the rotavirus infection is endemic in the country. The epidemiological summary of rotaviruses generated as of now clearly speaks on the importance of disease for the Indian livestock sector and emphasize on the need foran effective vaccine for growing bovine population in the country. During the last one decade, a few effective vaccines have become available to control this viral infection in humans, but still comprehensive and specific control measures for viral gastroenteritis are lacking in the domestic animal species, especially bovines. During the presentation an overview on the frequency of rotavirus infection and genotypes circulating in Indian bovine population, genetic diversity and evolution of rotaviruses in bovine population will be presented.

\section{Peste des petitsruminants vaccination strategies and control programme in India}

\section{Balamurugan, G. Govindaraj and Parimal Roy}

Email ID for Correspondence: balavirol@gmail.com; b.vinayagamurthy@icar.gov.in Indian Council of Agricultural Research-National Institute of Veterinary Epidemiology and Disease Informatics (ICAR-NIVEDI), Ramagondanahalli,, Post Box No. 6450, Yelahanka, Bengaluru 560064, Karnataka, India

PPR is enzootic in India as number of outbreaks has occurred in the past and now being occurring regularly, round the year and most frequently during the lean period throughout the country. In some Indian states viz. Andhra Pradesh, Karnataka, and Chhattisgarh the PPR outbreaks in sheep and goats have declined after implementing the strategic mass vaccination control programme. The decreased number of outbreaks in the recent past as well as changes in the disease severity patterns and distribution might be due to the effectiveness of vaccine, timely vaccination, circulation of a single lineage virus and most importantly effective planning and implementation of the vaccination programme. In overall, fixed strategies may not work for all the states or region or the countries. However, in the mass vaccination in pulse polio model covering entire population initially, followed by bi-annual vaccination in a predesignated stipulated period, covering the naïve young population of sheep and goats at least 3-4 years will have a tremendous impact on the control of PPR outbreaks in sheep and goats. Followed by vaccination on the migratory population at the check post or border regions of the states or inter-state border or in the place of entry or place of trade market of animal through transport from other states are to be targeted for mass vaccination as and when required. Sharing the experiences on the PPR vaccination strategies adopted by some of the states in India may motivate other Indian states or other countries of similar socio-economic and small ruminant rearing pattern to vaccinate and control PPR.

\section{Mechanisms of genome packaging in large DNA viruses and bacteriophages}

\section{Kiran Kondabagil}

Email ID for Correspondence: kirankondabagil@iitb.ac.in Department of Biosciences and Bioengineering, IIT Bombay

Proper segregation of chromosomes and their partitioning into daughter cells or capsids is a common problem faced by cellular and viral replicons. Most DNA viruses with genome size $>20 \mathrm{~kb}$ make use of an ATP-dependent process to pump the properly segregated unit-length genome into the preformed capsid. In dsDNA bacteriophages, a protein known as large terminase pumps the DNA at incredible rates of up to $2000 \mathrm{bases} / \mathrm{s}$ in an ATP-dependent manner. Single molecules studies have further shown that the bacteriophage T4 terminase motor is capable of generating forces of up to $80 \mathrm{pN}$ making it one of the most powerful biological motors. But, interestingly, our recent studies have shown that in large eukaryotic viruses such as Mimivirus that possesses a huge 1.2 Mb genome, a bacterialike chromosome segregation motor carries out this function. Some of the recent findings and fascinating features of these two types of packaging systems will be discussed.

\section{Host response to pathogen: transcriptome and proteome approach}

\section{Ravi Kumar Gandham, B.P. Mishra}

Email ID for Correspondence: gandham71@gmail.com ICAR - Indian Veterinary Research Institute, Izatnagar, Bareilly 243122

Peste des Petits Ruminants (PPR) is a viral disease of economic importance, causing an acute, highly contagious disease in small ruminants-goats and sheep. Peste Des Petits Ruminants Virus (PPRV), the causative agent of PPR belongs to the genus Morbillivirus, family Paramyxoviridae. The disease causes severe economic losses in terms of high mortality (up to $90 \%$ ) and morbidity (100\%) affecting the productivity of sheep and goats in endemic regions. 
Given its economic relevance and severity, the disease is classified as a World Organization for Animal Health (OIE) listed disease. Reports suggest that goats may be more susceptible with severe form of the clinical disease and pathology, than sheep. It has also been observed that the rate of recovery is lower in goats than in sheep. Contrasting reports suggest severe outbreaks of PPR in regions having large sheep populations, indicating that PPRV causes serious clinical disease in sheep as well. The host response to PPRV in the two species has never been investigated at transcriptome level. RNA sequencing data generated from PPRV infected sheep and goats PBMCs revealed interesting findings. Gene Ontology (GO) analysis identified important pathways related to immune response. Ingenuity Pathway Analysis (IPA) of differentially expressed genes revealed important upstream regulators involved in PPR disease pathogenesis. Protein interaction network revealed several key molecules as hubs. This is the first comprehensive work on deciphering the host response to PPRV.

\section{Trends in emerging and re-emerging viral diseases of pigs in India}

\section{Dilip Kumar Sarma}

Email ID for Correspondence: dksarma1956@gmail.com ICAR-National Research Centre on Pig, Rani, Guwahati-781131, Assam

Due to increasing demand for pork and pork products in India, the Indian pig sector needs significant changes not only in production but also in the entire pig value chain. Although changes are taking place, but there is less visibility in the growth due to various challenges. One of the major challenges is to address the increasing trends of emerging and re-emerging viral diseases. Porcine reproductive and respiratory syndrome (PRRS), the emerging viral disease in India was first appeared in Mizoram during 2013 is now spreading to other parts due to poor biosecurity and increasing movement of pigs and human. Increasing incidence of classical swine fever (CSF), the most important economic disease of pigs has been reported from the major pig producing states of India either due to poor vaccination coverage or increasing pathogencity of the isolates belonging to the new sub genotypes 2.1 and 2.2, which are reported in the recent field isolates of the virus from different parts of the country. Severe outbreak of CSF has also been reported recently in Mizoram. Foot and mouth disease which is another economically important disease of pigs is yet to be controlled in India, though efforts have been taken to control the disease in cattle and buffaloes. Other viral diseases like porcine circovirus type 2 (PCV2), porcine parvo virus (PPV) and rota virus infections which are almost endemic in India have been reported regularly and the phylogenetic analysis of the recent PCV and PPV isolates showed $99 \%$ homology with the Chinese strain of the viruses. Serological survey on the major viral diseases of pigs in Assam also showed prevalence of swine hepatitis $\mathrm{E}$ virus, besides the prevalence of PRRSV, CSFV, PPV, PCV2 and rota virus antibodies. Swine pox has also been reported recently from Assam and Meghalaya. Increasing incidence of all these viral diseases will adversely affect the growth of piggery sector in India unless the diseases are controlled by taking collective efforts. Improvement in the pig management practices, strengthening of the biosecurity level and early diagnosis of the diseases will also help to reduce the spread of the diseases. Preparedness on containing other exotic and zoonotic pig diseases is also essential for sustainable pig production in the country. An overview on the trends in emerging and re-emerging viral diseases of pig in India along with the strategies for their control will be presented in the paper.
Recent advances in diagnosis of rabies in animals and it's strengthening under OIE twinning programme in India

Shrkrishna Isloor, D. Rathnamma, Lekshmi J. Das, A. R. Fooks and S. Abdul Rahman

Email ID for Correspondence: kisloor@gmail.com

KVAFSU-CVA-Crucell Rabies Diagnostic Laboratory, Department of Microbiology, Veterinary College, KVAFSU, Hebbal, Bangalore560024

The laboratory-based diagnosis of rabies in animals is important for implementation of prophylactic measures. Diagnostic approaches based on conventional tests with special reference to Sellar's staining have a tendency to be unreliable. In the last three decades, the advances in immunology and molecular biology have enabled the development of highly sensitive, specific, user friendly techniques that could rapidly diagnose rabies with high accuracy. Presently, microscopic analysis of samples based on WHO/OIE approved Direct Fluorescent antibody Assay (DFA) is rapidly replacing Sellar's staining technique in several laboratories across the world for confirmatory diagnosis of animal rabies. In recent days, application of direct, rapid immunohistochemistry test (dRIT) in diagnosis of animal rabies is becoming popular as this uses a light microscope in contrast to DFA, independent of incubator and results are unambiguous. Another immunological method of detection of rabies virus antigen is recently developed Lateral flow assay. This assay has great potential as user friendly since it does not demand any laboratory equipment. Recently, nucleic acid detection-based assays with special reference to Reverse- Transcriptase Polymerase Chain Reaction (RT-PCR), Real time PCR and Loop Mediated Isothermal amplification (LAMP) are being employed either for detection of virus for and antimortem or post mortem confirmation of animal rabies and molecular epidemiological purposes. In India, several veterinary and medical institutions are involved in rabies related research activities with special reference to diagnosis and molecular epidemiology. In this context, we have taken a lead position at the national level in building up the human resource through national level training programme on application of monoclonal antibody based diagnostics such as dRIT and DFA for the detection of the rabies virus.. In view of the need to improve the global capacity for disease prevention, detection and control through better governance of veterinary services, the World organization for animal Health (OIE) has established a global programme 'OIE Laboratory Twinning Programme' whereby its OIE reference centres build expertise for the most important animal diseases including rabies) in priority regions through twinning with national laboratories In India, rabies is most often transmitted via the bite of dog. An unequivocal laboratory diagnosis of rabies is of immense importance for evolving effective and suitable preventive and control strategies. In this direction, the KVAFSU-CVA - Crucell Rabies Diagnostic Laboratory, Department of Veterinary College, Hebbal, Bengaluru established in 2013 has been recognized as a twinning partner. The project proposal, its aims and objectives and plan of action and other details will be discussed.

\section{Emerging viral pathogens in fish and shellfish aquaculture in India}

\section{K Riji john, M. Rosalind George, R. Dinesh}

Email ID for Correspondence: rijijohn@gmail.com Department of Fish Pathology and Health Management, Fisheries College and Research Institute, Tamil Nadu Fisheries University, Thoothukudi, Tamil Nadu

Aquaculture has been expanding rapidly around the world due to increased global demand for seafood, declining wild catches and 
improved culture techniques coupled with diversification of culture species. Despite some of the best management practices that are in vogue in scientific farming of fish and shellfish, aquaculture is still bogged down by incidences of diseases that are either due to opportunistic pathogens or arising due to biosecurity breaches. Among the different diseases affecting aquaculture, those due to viruses are by far the most deleterious and economically devastating. In India, one of the classic examples is of the emergence of white spot syndrome virus (WSSV) that decimated shrimp aquaculture in the nineties. Other viruses that are affecting shrimp farming in India include infectious hypodermal and hematopoietic necrosis (IHHNV), Laem-Singh virus (LSNV), hepatopancreatic parvovirus (HPV) and more recently infectious myonecrosis virus (IMNV). Viruses were found to cause considerable mortalities in both cultivable and ornamental fishes in India. From the first report of viral nervous necrosis from seabass caused by Lates calcarifer nervous necrosis virus (LCNNV) in the last decade, more viral agents were reported from fish. Koi ranavirus (KIRV), Cyprinid herpesvirus-2 (CyHV-2), Carp edema virus (CEV), Tilapia lake virus (TiLV), Similar damselfish virus (SRDV) and Snakehead rhabdovirus-like agent are the other viruses reported from different fish species. All reported viruses were associated with morbidity and mortality. Since the virus infected stocks are not possible to be recovered, quarantine, prophylaxis, biosecurity and early diagnosis are the most viable and often adhered management principles adopted in both fish and shrimp farming in India. Post-successful introduction of specific pathogen free (SPF) Penaeus vannamei, the crippled shrimp farming sector has grown leaps and bounds taking the total production of cultured shrimps from 76,000 tons in $2009-487,000$ tons in 2016. Strong regulatory measures and guidelines imposed by Coastal Aquaculture Authority towards management of transboundary and emerging diseases have been the major reasons for this success. Similar health management measures in both ornamental and cultivable fish culture would help the Indian aquaculture sector to progress further to achieve greater heights.

\section{Evaluation of antiviral and drug resistance mechanism of emetine}

\section{Sanjay Barua, Naveen Kumar, Thachamvally Riyesh, B.N. Tripathi}

Email ID for Correspondence: sbarua06@gmail.com National Centre for Veterinary Type Cultures, ICAR-National Research Centre on Equines, Hisar, Haryana

In this study in vitro and in ovo antiviral efficacy of emetine was studied against RNA and DNA viruses. Emetine was found to inhibit replication of RNA viruses; Newcastle disease virus (NDV) and peste des petits ruminants virus (PPRV) and DNA viruses; buffalo poxvirus (BPXV) and bovine herpesvirus type 1 (BHV-1). Emetine was found to primarily inhibit viral genome synthesis, though effect on viral entry was also observed in NDV and BHV-1. Furthermore, emetine was also found to significantly inhibit BPXV-induced pock lesions on chorio-allantoic membrane (CAM) along with associated mortality (embryonated SPF chicken eggs) in ovo. Similarly, emetine significantly delayed NDV-induced mortality in chicken embryos associated with reduced viral titers. Interestingly, no emetine-resistant mutants were observed upon long-term $(P=25)$ sequential passage of BPXV and NDV. We propose emetine as a broad spectrum antiviral agent with fewer tendencies in acquiring antiviral drug resistance.

\section{SERCA regulates paramyxovirus replication}

\section{Naveen Kumar, Sanjay Barua, Thachamvally Riyesh, Nitin Khandelwal, Krishan Dutt Rawat and Bhupendra Nath Tripathi}

Email ID for Correspondence: naveenkumar.icar@gmail.com National Centre for Veterinary Type Cultures, ICAR-National Research Centre on Equines, Hisar, India

We screened a library of host cell's kinase and phosphatase inhibitors. One of the inhibitor-sarco/endoplasmic reticulum $\mathrm{Ca}^{2+}$-ATPase (SERCA) inhibitor, when applied to peste des petits ruminants virus (PPRV) infected cells, resulted in reduced virus yield, suggesting SERCA (a host protein) is critically required for PPRV replication. With the help of a time-course assay, it was demonstrated that SERCA regulates localization of the viral proteins in infected cells (from cytoplasm to the plasma membrane of infected cells). Co-immunoprecipitation assay suggested no direct interaction between PPRV and SERCA. Similar findings were observed with another paramyxovirus, Newcastle disease virus (NDV). SERCA overexpression resulted in enhanced NDV replication and rescued the inhibitory effect of Thapsigargin on NDV replication which confirmed that SERCA is specifically required for paramyxovirus replication.

\section{Profile of Culicoides populations in livestock holdings in Tamil} Nadu with particular reference to bluetongue

\section{Y. Krishnamohan Reddy}

Email ID for Correspondence: kmreddy.y@tanuvas.ac.in Vaccine Research Centre - Viral Vaccines, Centre for Animal Health Studies, Tamil Nadu Veterinary and Animal Sciences University, Madhavaram Milk Colony, Chennai-600051, India

Bluetongue is one of the most prevalent infectious non-contagious diseases of sheep caused by Orbivirus of the Reoviridae family. The disease is transmitted by the bite of the midges of the genus Culicoides belonging to the family Ceratopogonidae. Severity of bluetongue infection depends on environmental conditions such as high rainfall, humidity and vector population. Thus, the disease occurrence gains momentum during the post monsoon season when the inclemental weather favours the abundance of vector population. The present study was designed for sampling Culicoides species in and around livestock premises to define both distribution and seasonality of the vector. Light suction traps were set up for collecting Culicoides from different agro climatic zones of Tamil Nadu. The LED Light suction traps were found to yield a good catch when compared to the sweep nets. In contrast, CDC traps were found to collect diversified Culicoides population that included different life stages of the vector. Use of UV light-suction traps were found to be more effective than the green light LED traps. Morphological identification of Culicoides was carried out using the wing pattern, abdominal characteristics and head and mouth parts as per the keys available for Culicoides classification. The predominant species identified from Tamil Nadu include C.imicola, C.oxystoma and C.peregrinus. Other species identified include C.clavipalpis, C.huffi, C. brevitarsis, C. peliliouensis, C.innoxius, C. palpisimilis, C.geminus, C.arakawae, C.actoni and C.fulvus. The authenticity of the vector identification was further confirmed by DNA barcoding that revealed 12 morphologically different species. Three bluetongue serotypes were detected in the Culicoides catch, which coincided with the prevalent serotypes detected in the affected sheep population. It can be concluded that effective Culicoides surveillance programme could be achieved by combining morphological and molecular 
identification techniques when ideal sampling procedures are carried out to trap Culicoides population in and around livestock holdings in order to minimize bluetongue virus transmission in susceptible geographical locations.

Isolation, identification, and characterization of inclusion body hepatitis (IBH) virus from poultry, India

Dashrath Shinde ${ }^{1}$, Anil Thormothe ${ }^{1}$, Santosh S Koratkar', Ashok Rajguru $^{2}$, Neeti Sharma ${ }^{1}$, Hermann Klein-Hessling ${ }^{2}$, Teguh Y. Prajitno ${ }^{2}$, Satish Tongoankar ${ }^{1}$, Vinay Rale ${ }^{1}$

Email ID for Correspondence: santosh.koratkar@ssbs.edu.in ${ }^{1}$ Symbiosis School of Biological Sciences (SSBS), Symbiosis International University (SIU), Knowledge Village, Gram Lavale, Taluka Mulshi, Dist. Pune 412115, India; ${ }^{2}$ Japfa Comfeed India Pvt. Ltd. 91, Sakore Nagar, Viman Nagar, Pune 411014, India

Avian adenoviruses are an adverse group of pathogens causing variety of diseases in poultry. Inclusion body hepatitis (IBH) is one of the most important re-emerging diseases worldwide. The aim of this study was isolation and molecular characterization of Fowl Adenovirus (FAdV) associated with IBH in India. A total 127 liver samples of IBH suspected broiler chickens were collected from different regions of India in 2015-2016. Liver samples were initially screened by histopathology and those that showed basophilic, intra-nuclear inclusion bodies microscopically were processed further for FAdV isolation. The molecular detection of FAdV was done by PCR using specific primers for hexon gene amplification. Of 127, 69 samples were found positive in PCR of which 28 samples were sequenced. The resultant sequences were compared with published sequence FAdV serotypes 1-12. The phylogenic tree analysis by ClustalW showed sequence similarity more than $94 \%$ with FAdV2 and $97 \%$ with FAdV12. The FAdV12 isolates were further confirmed by Restriction Enzyme Analysis (REA) using AspI restriction enzyme. The virus was isolated in Chick embryo Liver (CEL) culture derived from Specific Pathogen Free (SPF) and embryonated SPF chicken eggs inoculated by yolk sac route. The isolated virus was subsequently passaged up to 5th passage and finally, virus titration was carried out, TCID50 and EID50 titres were estimated. Pathogenicity of isolated FAdV was tested in SPF chickens and $100 \%$ mortality was observed by PID-6 in day-old SPF chicks. Liver and kidney were tested in histopathology for microscopic changes and IBH specific changes were found. Liver suspension was retested by PCR and found positive for hexon gene amplification. This study provides the evidence and explains the role of FAdV serotype12 responsible for causing outbreaks of IBH in broiler chicken in India.

Structural characterization of white spot syndrome viral enzymes involved in nucleotide synthesis via de novo pathway

\section{Sushil Kumar, Supriya Borah, Dileep Vasudevan}

Email ID for Correspondence: dileep@ils.res.in

Institute of Life Sciences, Bhubaneswar, Odisha State, India - 751023

White Spot Syndrome Virus (WSSV) is a devastating viral pathogen that results in serious economic losses due to infections caused in cultured penaeid shrimps and other crustaceans. This DNA virus codes for several enzymes, of which a good majority belong to pathways in DNA replication, synthesis and metabolism. During infection, viral enzymes involved in nucleotide synthesis and replication get expressed in large amounts in order to allow rapid proliferation of the virus. The sole WSSV enzymes that take part in viral deoxythymidine monophosphate
(dTMP) synthesis via de novo pathway are Deoxyuridine triphosphatase (dUTPase) and Thymidylate synthase (TS). Among these, dUTPase converts deoxyuridine triphosphate (dUTP) to deoxyuridine monophosphate (dUMP) and TS catalyzes the conversion of dUMP to dTMP. In the current work, we have completed the crystal structure of dUTPase and TS enzymes from WSSV. The dUTPase enzyme forms a typical trimeric structure and TS forms a functional dimer. We are in the process of studying complexes of these enzymes with inhibitors and we hope some of these potent inhibitors could be used for suitable anti-viral treatment modalities.

Exploring dsRNA for therapy of shrimp white spot syndrome virus (WSSV)

Subhendu Kumar Otta, P. Saravanan, C. Bala Amarnath, M. Makesh and S.V. Alavandi

Email ID for Correspondence: subhendu@ciba.res.in, oksubhen@yahoo.com

Aquatic Animal Health and Environment Division, ICAR-Central Institute of Brackishwater Aquaculture, 75, Santhome High Road, R. A. Puram, Chennai 600028

Shrimp Aquaculture is a multibillion dollar industry which substantially influences the economy of many countries. Disease often comes as a prime hurdle for this. White Spot Syndrome Virus (WSSV) has been constantly affecting as a single most important agent causing huge economic loss. Even after three decades since its emergence, it has not been possible to develop any preventive or treatment strategies for WSSV. As RNAi mechanism appears to be functional in shrimp, it provided hope in developing control strategies against this virus. We tried to explore the possibility of applying this technology for controlling WSSV. Out of three important WSSV genes (VP28, VP19 and rr1), dsRNA against VP28 was found to be more efficient in providing protection through injection mode of delivery. However, VP28 dsRNA was able to protect shrimp against WSSV challenge only when the dsRNA was injected to shrimp at least $24 \mathrm{~h}$ before challenge indicating its function as a prophylactic. VP28 dsRNA when conjugated with chitosan nanoparticle and supplied through feed for 10 days of continuous feeding, no protection was observed upon experimental challenge with WSSV either through injection or oral feeding. Alternatively, bacterially expressed VP28 dsRNA was administered through feed to shrimp and here also, it was not possible to obtain any protection against WSSV. In another trial, post larvae of Penaeus vannamei were immersed in different concentrations of bacterially expressed VP28 dsRNA for $1 \mathrm{~h}$ and subsequently reared in the laboratory. These were subsequently challenged with WSSV at different time intervals through oral feeding. No protection was observed in any of the bacterial concentrations or at any of the time period intervals post immersion. All these experiments indicate the limited efficacy in the application of dsRNA as therapeutic option in the control of WSSV in the shrimp rearing systems such as hatchery and farm.

Genetic analysis of viral interferon resistance (VIR) gene of Orf virus isolates from India reveals structural conservation of the protein in parapox viruses

Monu Karki, Amit Kumar, G. Venkatesan, Sargam Arya, M A Ramakrishnan and A B Pandey

Email ID for Correspondence: gnanamvirol@gmail.com Division of Virology, ICAR-Indian Veterinary Research Institute, Mukteswar, Nainital 
Orf virus (ORFV) is the prototypic member of genus Parapoxvirus of subfamily Chordopoxvirinae and family Poxviridae. ORFV is known for its unique strategy of encoding an array of immuno-modulating proteins and virulence factors as a result of its interaction with host cell. One of the important virulence factor is the viral interferon resistance gene (VIR) encoded by ORF020 from 5' end terminus of the genome. The present study focuses on the detailed genetic characterization and phylogenetic analysis of Indian ORFV isolates $(n=9)$ from different geographical regions of India. The gene was found to be highly conserved among all ORFV isolates used in the study (552 bp), whereas, a significant variation in gene length was observed in other members of family. Sequence analysis showed 92.4-99.8\% and $84.8-99.5 \%$ identity among the Indian ORFV isolates included in the study, at nt and aa level, respectively. Further, they share 92.0-99.1\% and 84.8-97.8\% identity with foreign ORFV isolates. Multiple sequence alignment showed that the encoded protein possesses many conserved residues among ORFV isolates. Comparative analysis showed that ORFV and PCPV isolates were more related to each other than BPSV and high variability was noted among the respective VIR gene from different members of family Poxviridae. Phylogenetic analysis shows more relatedness among Indian and Chinese ORFV isolates. ORF020 product (VIR) is known to bind interferon induced dsRNA dependent protein kinase (PKR) via one of its domain at C-terminal. The conserved C-terminus dsRNA binding domain and SUMOylation site, N-terminus Z-DNA binding domain, Asn-glycosylation sites and conserved cysteine residues etc. are the important characteristics of this protein. These features make this gene as an interesting candidate to be studied completely to explore its potential in development of diagnostics and anti-viral therapeutics in control of parapox virus infections.

\section{Efficacy of bacteriophage therapy of luminescent bacterial disease} in commercial shrimp hatcheries

\section{A. Satheesha, Vidya, R., Chougala, A.S., Baskaran, V., Thiyagarajan, S., Sujeet Kumar, Alavandi, S.V. and Vijayan, K.K}

Email ID for Correspondence: satheesha@ciba.res.in Aquatic Animal Health and Environment Division, ICAR-Central Institute of Brackishwater Aquaculture, Chennai, India- 600028

Mitigation of bacterial disease is the main challenge being faced in shrimp hatcheries, and attracts several inputs that may be detrimental to environment and consumers. Luminescent bacterial disease caused by bacteria such as Vibrio harveyi or $V$. campbelli is one of the major concerns in shrimp hatcheries. In the present work, we demonstrated application of cocktail of bacteriophage as biocontrol measure in effective control of luminescent bacterial infection in commercial shrimp hatcheries. We developed consortia of four bacteriophages from a pool of 42 isolates with highest host range among 370 luminescent bacterial isolates, comprising both $V$. harveyi and $V$. campbelli. Efficacy in controlling vibriosis was checked in the larvae of Penaeus vannamei by prophylactic treatment. Forty strains of highly pathogenic vibrios were challenged to nauplius-6-stage of the shrimp ( $P$. monodon). Prophylactic treatment with multiplicity of infection (MOI) of one provided significantly higher survival rate of 90-99\% against $48-0 \%$ survival in infected control tanks. The study was further conducted in three commercial $P$. vannamei hatcheries in larval rearing tanks of $10 \mathrm{~T}$ capacities. A MOI of 5 was followed for prophylactic treatment while 10 for therapeutic treatment. Phage prophylactic treatment significantly reduced the total vibrio count as well as luminescent bacterial count in the hatchery tanks. The larval survival at PL 7 stage was 35, 65 and $77 \%$ in phage treated tanks against 0,60 and $60 \%$ larval survival in the tanks with standard hatchery protocols. Therapeutic phage treatment was conducted in mysis 3 stage with an MOI of 10 and the treated tank showed improvement with survival with $36 \%$ at $\mathrm{PL}_{5}$ stage whereas all the tanks affected with luminescent bacterial disease were wiped out with complete larval mortality. This study has proved that phage therapy using consortia of selected broad spectrum phages can be a promising prophylactic as well as therapeutic biocontrol option for managing luminescent vibriosis in shrimp hatcheries.

\section{Status of PPR vaccination in Chhattisgarh state, India}

\section{Balamurugan', D. Muthuchelvan ${ }^{2}$, G. Govindaraj ${ }^{1}$, Gautam Roy $^{3}$, S Sowjanya Kumari ${ }^{1}$ Dheeraj Choudhary ${ }^{2}$, B. S. Mohanty ${ }^{1}$, K.P. Suresh ${ }^{1}$, K.K. Rajak ${ }^{2}$ D. Hemadri' ${ }^{1}$, Parimal Roy ${ }^{1}$}

Email ID for Correspondence: balavirol@gmail.com

${ }^{1}$ ICAR-National Institute of Veterinary Epidemiology and Disease Informatics (NIVEDI), Yelahanka, Bengaluru, Karnataka, India; ${ }^{2}$ Division of Virology, ICAR-Indian Veterinary Research Institute (IVRI), Mukteswar Campus, Nainital, Uttara khand, India; ${ }^{3}$ Directorate of Veterinary Services, New Raipur, Chhattisgarh, India

Peste des Petits Ruminants (PPR), is a highly infectious viral disease of small ruminants that causes significant production losses for sheep and goats owners in developing countries. In Chhattisgarh state of India PPR mass vaccination was carried out yearly since 2010-2011 to control PPR in sheep and goats. This study assessed the status of PPR vaccination in an epidemiological perspective by assessing postvaccination sero-conversion in the targeted population. As per stipulated guideline of control programme framework, $0.01 \%$ post vaccinated serum samples over a period of 6 years period were collected (14235 post-vaccination sera) randomly from 29 districts of state by the implementing authorities and were tested yearly for the presence of PPRV antibodies using competitive ELISA. In order to know the actual status of vaccination, 214 post-vaccination serum samples from 17 districts were collected along with epidemiological parameters through stratified random sampling methods by designed cross sectional study based on population during 2016-2017. Overall, the results from random sampling indicate that $84.22 \%$ of goats' population presents protective antibodies at a significant level against PPR when randomly assigned $0.01 \%$ sampling size was assessed. However, the sero-protection percentage was only $49 \%$ during the year 2016-2017 when stratified random sampling procedure was applied. Further, on Chi square analysis, statistically significant difference in seroconversion rate was observed between the years 2010-2016 ( $\left.\chi^{2} 151.9\right), 2010-2017\left(\chi^{2} 191.0\right)$ and, between 2010 and 2016 and 2016-2017 ( $\left.\chi^{2} 339.0\right)(P<0.001)$. Moreover, the significant difference was observed in positivity across age groups $\left(\chi^{2} 8.66\right.$, $P<0.01)$ and not between species, breed, sex, body condition, history of vaccination and earlier infection status, deworming practice and antibiotics use. Results demonstrate that PPR sero conversion levels in the study areas was lower (49\%) during 2016-2017 than the recommended $\sim 70 \%$ that prevents virus circulation in endemic and high risk areas. Sero-negative animals and presumably many of the animals in the study areas were therefore not protected against future PPR outbreaks, though no outbreaks were reported since 2014. This indicates, annual vaccination strategy adopted in Chhattisgarh has not been effective in reaching the desired protection levels. Hence, mass vaccination activities need to continue for 2-3 years by targeting endemic areas in bi-annual mode to achieve the desired protection level. 
Phage mediated virulence in emerging bacterial pathogen Vibrio campbellii

\section{Sujeet Kumar $^{1}$, S.V. Alavandi ${ }^{1}$, K.N. Viswas ${ }^{2}$, Vidya Rajendran ${ }^{1}$ and K.K. Vijayan ${ }^{1}$}

Email ID for Correspondence: sujeetmicrobiol@gmail.com

${ }^{1}$ ICAR-Central Institute of Brackishwater Aquaculture; ${ }^{2}$ ICAR-Indian Veterinary Research Institute

Vibriosis is the most serious bacterial infection of shrimp hatchery, causing up to $100 \%$ mortality during mysis and early postlarval stages. Luminescent Vibrio such as Vibrio harveyi has been found mostly responsible for vibriosis. In recent years $V$. campbellii has also been associated with vibriosis infection and now being considered as an emerging shrimp pathogens. To identify the virulence markers, a pathogenic luminescent Vibrio isolate LB1, isolated from gills of vibriosis affected brooder, was selected for the study. The isolate was characterised by phenotypic and molecular methods including hemolysin based multiplex PCR, and sequencing of $16 \mathrm{~S}$ rRNA and housekeeping rpoD gene. The $16 \mathrm{~S}$ gene sequence shared $99 \%$ similarity with $V$. parahaemolyticus, $V$. harveyi and $V$. campbellii. However, the multiplex PCR using hemolysin gene showed characteristic 328 bp product of $V$. campbellii. Further, the BLAST analysis of rpoD gene sequence showed $99 \%$ similarity with $V$. campbellii in comparison to $95 \%$ similarity with $V$. harveyi. The level of pathogenicity of the isolate was confirmed in white leg shrimp, $P e$ naeus vannamei postlarvae (PL8) by immersion method with a late

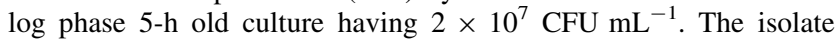
produced $90 \%$ mortality. The bacterial supernatant had higher level of protease activity (112 U) which was largely inhibited by para hydroxy mercuribenzoic acid (PHMB), a cysteine protease inhibitor. The mitomycin induction assay indicated the presence of lysogenic phage with significant reduction in $\mathrm{OD}_{600}$ compared to uninoculated group. The cesium chloride density gradient produced two white bluish bands at 1.3-1.5 density. The transmission electron microscopy revealed the presence of lysogenic phages of a siphovirus and a filamentous phage. Genomic and bioinformatic analysis indicated that such phages carry toxins which could be playing role in the virulence of emerging aquatic animal pathogen such as $V$. campbellii and $V$. harveyi.

\section{Tilapia lake virus (TiLV) an emerging threat to tilapia farming in India}

\section{Poornima, S.V. Alavandi and K.K. Vijayan}

Email ID for Correspondence: poornima@ciba.res.in ICAR-Central Institute of Brackishwater Aquaculture, Chennai - 600 028, India

Tilapia is widely-cultured third most important fish species after carps and salmon in the world. The worldwide Tilapia production is estimated 6.4 million metric tons (MMT) in 2015 valued at USD 9.8 billion. Genetically Improved Farmed Tilapia (GIFT) is one of the key candidate species suitable for aquaculture in India, owing to its fast growth, quick adaptation under diverse farming systems, tolerance to wide range temperatures and low cost farming. Recently, the import of Oreochromis niloticus and tilapia farming has been approved with reframed guidelines developed by the National Committee on Introduction of Exotic Aquatic Species into Indian Waters. It is generally presumed that the Tilapia are disease-resistant, its cultivation is profitable and risk-free. However in the recent past, Tilapia lake virus (TiLV), an emerging highly contagious disease has been posing a serious threat to both farmed and wild tilapia, with cumulative mortalities reaching up to $80-90 \%$ in affected tilapia fish populations. The recent episode of TiLV is the first-ever serious disease, rampantly reported in tilapia aquaculture and is an emerging peril, causing a high jeopardy to the global tilapia industry due to economic loss caused by concomitant mortalities in farmed tilapia. TiLV has been tentatively placed as a novel virus in the Family Orthomyxoviridae. Since the first discovery of the virus in 2014, till date, TiLV has been reported from five countries viz., Colombia, Ecuador, Egypt, Israel and Thailand. However, implicit geographic distribution of TiLV may be more extensive than reported. In recent times TiLV has been detected in few states in India, which alarms tilapia production systems in the country. Intensification of aquaculture without proper biosecurity measures and imprudent live aquatic animal trade poses high risk to tilapia farming. Currently, there are no methods to control TiLV. General biosecurity measures and best management practices should be in place to minimise its spread. Horizontal spread is an important route of transmission. There is a significant risk of TiLV being translocated through the movement of infected live tilapias in the absence of appropriate biosecurity measures. Hence, while translocating live tilapia one should be vigilant and take appropriate risk management measures such as diagnostic testing of stocks especially of unexplained mortalities to reduce further spread of the disease. There is a need to examine the tilapia farming holistically, from source of broodstock, seeds, their pathogen status, etc., to identify the source of infection. The seeds/fingerlings before stocking must be tested and ensured to be free from TiLV. Brood fish must undergo quarantine procedures before shipment and post arrival and testing for all the OIE-listed diseases including TiLV must be carried out. Coordinated strategies from all stakeholders including fish farmers, state and central Governments would help effective management of this new threat to tilapia farming in the country.

Prevalence of white spot syndrome virus (WSSV) and Infectious hypodermal and haematopoietic necrosis virus (IHHNV) in black tiger and Indian white shrimp broodstocks in India

Jagadeesan, V. ${ }^{1}$, Ezhil Praveena, P. ${ }^{1}$, Vinay Kumar, K. ${ }^{2}$, Biju, I.F. ${ }^{3}$, Jithendran, K.P. ${ }^{1}$, Alavandi, S.V. ${ }^{1}$ and Vijayan, K.K. ${ }^{1}$

Email ID for Correspondence: epraveena@ciba.res.in

${ }^{1}$ Aquatic Animal Health and Environment Division, ICAR- Central Institute of Brackishwater Aquaculture, 75, Santhome High Road, R. A. Puram, Chennai, Tamil Nadu, India- $600028 ;{ }^{2}$ Nutrition, Genetics and Biotechnology Division, ICAR- Central Institute of

Brackishwater Aquaculture, 75, Santhome High Road, R. A. Puram, Chennai, Tamil Nadu, India- $600028 ;{ }^{3}$ Crustacean Culture Division, ICAR- Central Institute of Brackishwater Aquaculture, 75, Santhome High Road, R. A. Puram, Chennai, Tamil Nadu, India- 600028

Indian shrimp farming sector has witnessed substantial growth in the last 8 years after the introduction of specific pathogen free (SPF) Pacific white shrimp for aquaculture India. The present study was conducted to find out the prevalence of major shrimp pathogens such as white spot syndrome virus (WSSV) and infectious hypodermal and haematopoietic necrotic virus (IHHNV) in the two major species of India. Wild Penaeus monodon obtained during 2010-2014 ( $\mathrm{n}=231$ ) from various landing centers on the west coast at Gujarat, Ratnagiri, Mangalore and Kollam, on the east coast at Tuticorin, Chennai, Visakhapatnam, Kakdwip, and Port Blair (A and N Islands). The study indicated a very high prevalence of as much as $52 \%$ of the shrimps had either WSSV and/or IHHNV infection. 6\% of these black tiger shrimp broodstock had WSSV, and as high $38 \%$ of shrimp had IHHNV alone, while $8.2 \%$ had the mixed infection of both viral pathogens. It was observed that IHHNV was more prevalent in West 
coast and $\mathrm{A}$ and $\mathrm{N}$ Islands than in the East coast of India. A similar study was conducted during the year 2016-2017 in Indian white shrimp, Penaeus indicus $(\mathrm{n}=658)$, collected from Kollam on the west coast and Chennai and Odisha on the east coast. WSSV infection was detected in as high as $39.5 \%$ of $P$. indicus, and $6.1 \%$ of the animals had IHHNV infection. The study indicated that the prevalence of WSSV was significantly high in native Indian white shrimp broodstock. It was interesting to note that the prevalence of IHHNV was high in wild black tiger shrimp. IHHNV infection in $P$. monodon is a chronic, non-lethal disease. The high prevalence of IHHNV in $P$. monodon in the present study may have a bearing on the IHHNVrelated sequences being integrated into the genome of $P$. monodon. The black tiger shrimp breeding programmes must ensure freedom from IHHNV to prevent the occurrence of stunted growth problems during grow-out culture.

Laboratory rodent models for understanding pathogenesis of foot and mouth disease virus: histopathological analysis of suckling Wistar rat tissues

Tamil Selvan, R.P ${ }^{1}$, Krishnamoorthy, $\mathbf{P}^{2}$, Patel, N.K.P', Bhanuprakash, $\mathrm{V}^{1}$, Patel, B.H.M ${ }^{1}$ and Sanyal, $\mathrm{A}^{\mathbf{1}}$

Email ID for Correspondence: tamil.selvan@icar.gov.in ${ }^{1}$ ICAR-Indian Veterinary Research Institute, Regional Campus, Hebbal, Bengaluru-560024, Karnataka, India; ${ }^{2}$ ICAR- National Institute of Veterinary Epidemiology and Disease Informatics, Post Box No.6450, Ramagondanahalli, Yelahanka, Bengaluru560064, Karnataka, India

Foot and Mouth disease is the most important disease of cloven hooved domestic and wild animals and causes huge economic loss to the dairy farmers in India. The mechanism underlying the pathogenesis of Foot and Mouth Disease virus (FMDV), the causative agent of FMD, is not studied fully and understanding the same in natural host is difficult due to high cost involved. In the present study, the experimental infection of FMDV in suckling Wistar rat was attempted by five consecutive passages and result of inoculation was studied in various organs.

The Wistar rat pups (3 day old) were inoculated with cattle adapted Indian vaccine strain of FMDV. The rat pups were observed for presence of clinical signs and sacrificed $72 \mathrm{~h}$ post experimental infection. On gross examination, organs revealed no observable changes. Histo-pathological examination of heart revealed the striated skeletal muscle with striations in infected and control pups. Pancreas of FMDV infected group showed the presence of acinar glands with blue staining nucleus at periphery of the cell, cytoplasm filled with pink staining secretory granules and appeared similar to that of the control rat pup. Liver revealed normal architecture of hepatocytes with vesicular nucleus and arranged in cords with congestion of blood vessels and central vein. There was no change in the FMD virus inoculated suckling Wistar rat tissues when compared to the control rat pup. Thus, it can be concluded that FMDV induces no visible and microscopic changes in suckling Wistar Rat pups when inoculated by intra muscular route and attempts should be made to induce experimental infection by means of inoculation by intra-peritoneally or intra-cerebrally or subcutaneously or intra-dermally in foot pad region as attempted for Guinea pig or by use of cell culture adapted virus or by using other rat strains.
Financial impact of PPR in sheep and goats in Karnataka

Govindaraj G, Afrin Zainab Bi, Chaitra, H.R., Mohanty B.S., Balamurugan, V. Manjunatha Reddy G.B., Yogisharadhaya, R. and Parimal Roy

Email ID for Correspondence: mggraj74@gmail.com ICAR-National Institute of Veterinary Epidemiology and Disease Informatics, Post Box 6450, Yelahanka, Bengaluru - 560 064, India

Peste des petits ruminants (PPR) is one of the acute febrile and economically important viral diseases in sheep and goats, with mortality and morbidity rates as high as 90 and $100 \%$, respectively. In the present study multistage random sampling technique was adopted for the primary survey. The data was collected through face-to-face personal interview method from 350 sheep and goats rearing households in three districts (Kolar, Bangalore rural and Bagalkote) of Karnataka state during 2016-2017. The deterministic mathematical models were used to assess the visible financial loss due to PPR in small ruminants. The results revealed that the incidence and mortality levels was quite high in goats $(11.72,4.66 \%)$ than in sheep $(9.20$, $3.83 \%$ ) with the overall levels of 9.68 and $3.99 \%$, respectively during the survey period. The estimated financial loss per animal due to mortality, distress sale, weight reduction, treatment and opportunity cost of labour due to PPR using the deterministic models was Rs. 3225 , Rs. 2769 , Rs. 1113 , Rs. 139 and Rs. 16 respectively. The above findings revealed that PPR is an important disease in small ruminants in terms of physical and financial disease burden on the farmers who depend on sheep and goats for their livelihood. Despite mass vaccination under PPR control Programme (PPR CP), the disease was reported in the state during the survey period indicating the need for strengthening the programme especially through bi-annual vaccination, increased coverage, and timely vaccination of the population along with vaccine supply chain maintenance for effectiveness of vaccine at field level to combat the disease.

Comparative efficacy of nanoparticle based delivery of recombinant proteins VP28 and VP39 against WSSV infection in Litopenaeus vannamei

Akhila Dharnappa Sannejal ${ }^{1}$, Srinivas Muthalik ${ }^{2}$, Praveen Rai ${ }^{1}$ and Indrani Karunasagar ${ }^{1}$

Email ID for Correspondence: akhila@nitte.edu.in

${ }^{1}$ Nitte University Centre for Science Education and Research, Paneer Campus, Deralakatte, Mangaluru, India; ${ }^{2}$ Manipal School of Pharmacy, Manipal University, Manipal, India

White spot syndrome virus (WSSV) has been a major concern since it becomes one of the most dreadful crustacean viral pathogen. It makes a huge economic loss to the shrimp aquaculture industry after its inception in Taiwan. Since shrimp possess innate immunity, there is no effective preventive measure to treat WSSV to date. Although vaccination helps in preventing viral disease outbreaks in shrimp culture, the usage of oral vaccines has a major constraint for its potentiality due to improper delivery to the target tissue. The efficacy of chitosan nanoparticle conjugated recombinant proteins against WSSV were investigated on Litopenaeus vannamei by targeting the envelope proteins VP28 and VP39. The PCR amplified ORFs from the viral DNA were cloned and expressed in Escherichia coli cells followed by conjugating with chitosan nanoparticles. Feed coated with VP28 and VP39 chitosan complex were fed for a period of 2 weeks and challenged for WSSV. Results showed a relative percentage survival (RPS) of 80 and 90 for VP28 and VP 39 respectively, after 10 days of infection. The present study showed the significance 
of the application of recombinant proteins encapsulated with chitosan nanoparticles as the best therapeutic strategies in commercial shrimp culture.

\section{Selective packaging of antibiotic resistance determinants in induced prophage fractions}

\section{Juliet Roshini Mohan Raj and Indrani Karunasagar}

Email ID for Correspondence: julietm@nitte.edu.in

Nitte University Centre for Science Education and Research, Nitte University, Derelakatte, Mangaluru, India -575 018

Ecological role of bacteriophages has become a matter of great interest worldwide. Phages are widely distributed in locations populated by bacterial hosts, such as soil or the intestines of animals and it is estimated that there are about $10^{31}$ phage particles in the biosphere Antimicrobial resistance has been a key area of research over the recent years and molecular mechanisms underlying this are in the forefront. It is now established that phages in the environment carry resistance genes and enable their spread. The occurrence of resistance determinants in phage fractions has not been recorded as yet as a random event or a site-specific excision. The aim of this study was to address this mechanism in different environments where antibiotic resistant bacteria are present. In this study, 72 bacterial isolates from different environmental sources that carried resistance determinants (bla ${ }_{\mathrm{CTX}-\mathrm{M}}$, bla $\mathrm{T}_{\mathrm{TEM}}$, tet, sul and qnr genes) were treated with physical and chemical stress conditions for prophage induction. The phage DNA thus obtained was tested for resistance genes to estimate the ARDs carried by temperate phages and thus estimate site-specific excision. The bla ${ }_{\text {СтX-м }}$ genes were observed to be extremely mobile with the ARD being packed in prophage fractions in $72 \%$ (24 of 34) of the isolates followed by bla tetracycline resistance determinants, the tetA gene was in the inducible phage fraction in $33 \%$ of the isolates. The selective packaging of only certain resistance determinants in phage fraction was observed in isolates harbouring multiple resistance determinants. This study highlights the role of temperate phages in antimicrobial resistance transfer. Many domestic and commercially used chemicals can act as prophage inducing agents and hence there is a need for antimicrobial stewardship programme and the development of strategies to reduce antimicrobial gene dispersion in the environment.

\section{Poster Presentation}

\section{Isolation and characterization of Aeromonas phages}

\author{
Ajay Yannangur Muninarayanappa ${ }^{1}$, \\ Narasimhamurthy Yannangur Gummanna ${ }^{1}$, \\ Dechamma Mundanda Muthappa ${ }^{2}$, \\ Puneeth Thadooru Goolappa $^{1}$, Suresh Thangavel ${ }^{1}$, \\ Venugopal Moleyur Nagarajappa ${ }^{1}$ and \\ Girisha Shivani Kallappa ${ }^{1}$
}

Email ID for Correspondence: skgirisha@gmail.com

${ }^{1}$ Department of Fisheries Microbiology, College of Fisheries, Mangalore- 575 002, India; ${ }^{2}$ UCD Center for Molecular Innovation and Drug Discovery, University College, Dublin

Four lytic phages namely $1 \mathrm{~F}, 1 \mathrm{~J}, 25 \mathrm{G}$ and $25 \mathrm{~N}$ specific to Aeromonas spp. were isolated from river and creek waters by spot assay. Confirmation, propagation and determination of phage titers were carried out by soft agar method. Preliminary characterization of isolated phages showed narrow host range without cross reacting to non- Aeromonads, less sensitive to chloroform treatment and more sensitive at storage temperature of $45^{\circ} \mathrm{C}$. Transmission electron microscopy analysis assigned all the phages into the family $M y$ oviridae. The protein banding pattern of four phages was generated by SDS-PAGE and their molecular weight was in the range of $25-70 \mathrm{kDa}$. Aeromonas phages $25 \mathrm{~N}$ and $25 \mathrm{G}$ showed similar banding pattern with one major band with molecular weight of $22 \mathrm{kDa}$ and many minor protein bands, whereas in $1 \mathrm{~F}$ and $1 \mathrm{~J}$ showed similar bands with two minor bands at $13 \mathrm{kDa}$ and $17 \mathrm{kDa}$ and one major band with molecular weight of $24 \mathrm{kDa}$.

Development and characterization of Vero-goat SLAM cells constitutively expressing goat-SLAM receptor for replication efficacy of peste-des-petits-ruminants virus

\section{Ajay Kumar Yadav, Mukesh Bhatt, Ashok Kumar, Soumendu Chakravarti, D. Muthuchelvan, Praveen Kumar Gupta, Rabindra Prasad Singh and Kaushal Kishor Rajak}

Email ID for Correspondence: dr.ajayyadav07@gmail.com; kaushalvirol@gmail.com

Biological Products Division, ICAR-Indian Veterinary Research Institute, Izatnagar, Bareilly, U.P. (India), 243122

Peste-des-petits-ruminants virus (PPRV) falls under genus Morbillivirus of family Paramyxoviridae. PPRV is responsible for the economically important disease PPR in small ruminants (sheep and goats), and plays a crucial role in farmers'/entrepreneurs' income. Conventional cell line may or may not be helpful in virus isolation and supportive for efficient replication. To make cells more sensitive for virus replication, availability of cellular receptors is important. Signaling Lymphocyte Activation Molecule (SLAM/CD150) supports the entry of all the morbilliviruses and is present on activated $\mathrm{B}$ and $\mathrm{T}$ cells, immature thymocytes and B95a cells. In order to develop Vero cell line constitutively expressing SLAM receptor, full length SLAM receptor gene of goat (Capra hircus) from mitogen (ConA, $10 \mu \mathrm{g}$ / $\mathrm{mL}$ ) stimulated PBMCs (48 h post stimulation) was amplified with expression primer having FLAG tag signal sequence. The amplified product was directionally cloned into pcDNA3.1 (+) mammalian expression vector and confirmed by sequencing. The lineralised cassette of pcDNA3.1(+) having goat SLAM was transfected in Vero cells using Lipofectamine 3000 (M/s Invitrogen). G418 (neomycin) at $600 \mu \mathrm{g} / \mathrm{mL}$ was used for selecting the positive clone. Non-transfected Vero cells with and without antibiotic pressure were also kept as cell control and antibiotic controls, respectively. Transfected Vero cells (Vero-goat SLAM) could resist the antibiotic selection pressure, where as non-transfected Vero cells under antibiotic pressure died completely. Antibiotic resistant clones were expanded from plate (6well) to flask $(25 \mathrm{~cm} 2)$, maintained under antibiotic pressure in eagle's minimum essential media (EMEM) having $2 \%$ foetal calf serum. The SLAM gene transcript was checked by RT-PCR in Verogoat SLAM cells at 3rd, 4th and 5th passage level. Goat specific SLAM transcript in the transfected cells was detected; however there was no transcript in non-transfected healthy Vero cells. Further characterization at protein level is underway. 
Activity of bacteriophages against biofilm forming Staphylococcus aureus

\section{Anoopkrishna K, Rajeshwari Vittal, Indrani Karunasagar and Juliet Roshini Mohan Raj}

Email ID for Correspondence: anoopkrishna.k7773@gmail.com Nitte University Centre for Science Education and Research, Nitte University, Deralakatte, Mangaluru, Karnataka, India-575018

Staphylococcus aureus is an opportunistic pathogen with clinical manifestations ranging from minor skin infections to fatal diseases. Biofilms are common adaptations which permit bacteria to colonise in hostile environments and are considered to be a major problem for antibiotics and biocides. Increasing reliance on indwelling medical devices, which are inherently susceptible to biofilm-mediated infections, has contributed to unacceptably high rates of nosocomial infections, placing a strain on healthcare budgets and increasing the need for alternative therapeutic measures.

The present study aimed at isolating bacteriophages and determining their activity on biofilm forming S. aureus. Environmental samples were collected from different locations in and around Mangaluru, India. Of 35 samples collected, 21 phages active on $S$. aureus ATCC 29213 were isolated from 17 samples. Four of the 21 phages were propagative on the host. The four phages were characterised based on their growth kinetics and molecular characterisation by RFLP. Activity of these phages on planktonic forms and biofilms was determined by CFU drop assay and crystal violet biofilm assay respectively. Planktonic cells of $S$. aureus were found to be sensitive to phages PD1, PE1 and PE2. PE2 was able to result in a CFU drop only at high phage concentrations of $10 \mathrm{MOI}$ and $1 \mathrm{MOI}$. Decrease in the number of viable cells was seen even when lower concentrations $(<0.01$ MOI $)$ of phages PD1 and PE1 were used. Phages PD1 and PE2 were efficient in preventing biofilm formation as well. This study indicates that the bacteriophages isolated can be developed as an alternative tool for the medical interventions.

\section{Role of chicken cyclophilin a in new castle disease virus replication}

\section{Sachin Kumar, Vishnu Chauhan, Aparna K. Mohan}

Email ID for Correspondence: aparnamohan9@gmail.com Department of Biotechnology, Indian Institute of Technology Guwahati, Guwahati, Assam, India - 781039

Newcastle disease is a highly contagious viral disease of domestic poultry. It is characterised by digestive, respiratory and/or nervous signs. Therefore, the reason to enhance resistance to NDV infection in domestic flocks is quite clear both scientifically and economically. Cyclophilin A (Cyp A) belongs to a family of highly conserved and ubiquitous proteins known as cyclophilins (CyP). In addition to its role in protein folding and assembly, CyPA plays a critical role in the successful replication of viruses such as human immunodeficiency virus (HIV), hepatitis C virus (HCV), hepatitis B virus (HBV), etc. However, CyPA possesses a repressive effect on the replication of some viruses like Influenza A virus and rotavirus. In the present study, the role of chicken Cyp A in NDV replication has been investigated. Real-time PCR results reveal the differential expression of ChCyp A after NDV infection. Plaque assay confirmed a decrease in viral titre of NDV when ChCypA was overexpressed in Chicken Embryo Fibroblast cells. The viral protein interacting partner of ChCyp A was identified at the vivo level using coimmunoprecipitation assay. The present data demonstrates that chCypA possesses anti-NDV activity which allows the consideration of genetic improvement for resistance to NDV in chickens.
Antiviral activity of pcDNA-interferon lambda-3 (pcDNA-IFN $\lambda 3$ ) against foot-and-mouth disease virus (FMDV) infection

Barathiraja S, Gangadhara PAV, M. Sedhumadhavan, Brijesh kumar, Aniket Sanyal, Umapathi V, Dechamma HJ and G.R. Reddy

Email ID for Correspondence: barathirajasnew@gmail.com FMD(R) Lab., ICAR-Indian Veterinary research Institute, Hebbal, Bengaluru-560024, Karnataka, India

Foot-and-mouth disease (FMD) is highly contagious and economically important disease affecting cloven hoofed animals worldwide. The disease has been identified as the most important constraint to international trade in animals and animal products. It is endemic in India and the control measures are carried out by intensive vaccination programmes. Currently used BEI inactivated oil adjuvant vaccine gives sero-protection only after 7 days in the vaccinated animals. Hence, there is a need to develop a vaccine/therapeutic which can be used to control the disease at early stage of infection. In this present study, bovine IFN $\lambda 3$ gene was cloned in pcDNA vector (pcDNA-IFN $\lambda 3$ ) and evaluated for its antiviral activity against FMDV infection in vitro and in vivo. Foetal bovine kidney (LFBK) cells transfected with pcDNA-IFN $\lambda 3$ had shown significant reduction in viral load at $0.01 \mathrm{MOI}$ of FMDV serotype ' $O$ '. The observed Percent Inhibition (PI) was $96.10 \%$ in LFBK cell line. DNA construct, pcDNA-IFN $\lambda 3$ was also analyzed for in vivo antiviral activity using guinea pigs, where pcDNA-IFN $\lambda 3$ was administered $24 \mathrm{~h}$ before virus inoculation. Animals were observed continuously for 7 days. About $33.33 \%$ of animals got protected with pcDNA-IFN $\lambda 3$ treatment in comparison to none of the animals in control groups. There was also delay in virus infection in some of the animals as the protection rate was slightly decreased from day 5 to day 7 . The present study shows that pcDNA-IFN $\lambda 3$ shows antiviral activity in vitro and in vivo conditions. The study further shows that bovine IFN $\lambda 3$ delays the disease progress in guinea pigs.

Transcriptomic profiling of antiviral immune genes in lymphoid organ of white spot syndrome virus infected tiger shrimp, Penaeus monodon

Mundanda Muthappa Dechamma ${ }^{1}$, Kogaluru Shivakumaraswamy Santhosh ${ }^{2}$, Rajeish Moger', Kamalabhavan Maniyan Madhu' ${ }^{2}$, Volker Gurtler ${ }^{3}$, Indrani Karunasagar ${ }^{2}$

Email ID for Correspondence:

dechamma.mundandamuthappa@ucd.ie

${ }^{1}$ Department of Fisheries Microbiology, Karnataka Veterinary, Animal and Fisheries Sciences University, College of Fisheries, Mangalore-575002, India; ${ }^{2}$ Nitte University Centre for Science Education and Research, Nitte University, Paneer campus, Deralakatte, Mangalore-575018, India; ${ }^{3}$ School of Applied Sciences, Building 223, Level 1, Bundoora Campus RMIT University, PO Box 71, Bundoora, 3083

Transcriptomic analysis has emerged as a revolutionary tool in understanding host-pathogen interaction by examining disease response, defense strategies and developmental activities. This can reveal the functional genomics and molecular constituent of an organism against the infection. Hence the transcriptomic study along with real time PCR analysis was employed in the present study in revealing the differential gene expression of different antiviral immune genes during the white spot syndrome virus (WSSV) infection in Penaeus monodon (black tiger shrimp). Shrimps devoid of virus were injected with WSSV and TN buffer. Lymphoid organ from 
$12 \mathrm{~h}$ post infected animals were taken for further studies. The transcriptomic response of shrimp compared with control animals revealed the differential expression of potent genes when infected with WSSV. Transcripts derived from paired end sequencing of lymphoid organ of WSSV infected Penaeus monodon resulted in 40 million reads of assembled transcripts. Pair wise analysis of these transcripts resulted in 28160 usable transcripts of which 201 were found to be differentially expressed (p-value $<0.01$ ) where 62 transcripts were up-regulated and 18 transcripts were down-regulated in WSSV infected sample. Assembled transcripts were annotated using Contig Annotator Pipeline (CANoPI) software. Overall, 63 transcripts were found to be differentially expressed (p-value $<0.01$ ) with 45 transcripts were up-regulated and 18 transcripts were down regulated. The findings suggest that certain potent immune genes in tiger shrimp which are differentially expressed during the viral infection. The transcriptome data generated in this study will be extremely valuable in the improved understanding of host response to WSSV infection.

\section{Isolation and characterization of phages specific to Vibrio parahaemolyticus}

\section{Dilip Lakshman, Mukesh Yannanguru Krishnappa, Puneeth Thadooru Goolappa, Suresh Thangavel,} Venugopal Moleyur Nagarajappa and Girisha Shivani Kallappa

Email ID for Correspondence: skgirisha@gmail.com Department of Fisheries Microbiology, College of Fisheries, Mangalore- 575 002, India

The bacteriophages are ubiquitous in nature and exist in all the ecosystems where their specific host bacteria are also found. In this study, three lytic phages specific to Vibrio parahaemolyticus were isolated from clam sample, shrimp farm water and shrimp farm sediment samples and were designated as VPP-2, VPP-5 and VPP-11 respectively by spot assay method. Confirmation, propagation and determination of phage titer were carried out by soft agar method. Comparison of protein profiles of isolated phages by SDS-PAGE revealed that bands produced were unique to each phage. VPP-2, VPP-5 and VPP-11 had a major band in the range of 70, 40 and $35 \mathrm{kDa}$ respectively. Isolated bacteriophages found to be broad host specific without cross reacting to non-Vibrios with VPP-2 having broad host range compare to VPP-5 and VPP-11. Storage stability studies of phages indicated that the titer of the VPP-2 and VPP-5 phages were almost relatively stable up to 60 days at eight different temperatures viz $-80,-20,0,4,20,30,37$ and $50{ }^{\circ} \mathrm{C}$. But the phages were more stable between the temperatures ranges of -80 to $4{ }^{\circ} \mathrm{C}$ compared to 20 to $50{ }^{\circ} \mathrm{C}$ temperature range. Chloroform stability test showed that VPP-2 was relatively stable in nature when compared to VPP-5 phages and VPP-11 was less stable than other two phages.

\section{Comparative sequence analysis of L1R myristoylated protein of capripoxviruses reveals structural homogeneity among poxviruses}

\section{Monu Karki, Amit Kumar, Gnanavel Venkatesan, Sargam Arya and A. B. Pandey}

Email ID for Correspondence: gnanamvirol@gmail.com Division of Virology, ICAR-IVRI, Mukteswar 263 138, Nainital, Uttarakhand, India

Sheeppox virus (SPPV) and goatpox virus (GTPV) are members of Capripoxvirus genus of Poxviridae family, responsible for important contagious diseases endemic to Indian sub-continent, Central and Northern Africa and the Middle East. The present study describes the sequence features and phylogenetic analysis of the L1R gene of sixteen capripoxvirus (six sheeppox virus and nine goatpox virus) isolates from India. L1R is a myristoylated protein responsible for virion assembly. Being present on intracellular mature virion (IMV) surface, it is also a potent target for eliciting neutralizing antibodies. Sequence analysis of capripoxvirus L1R gene revealed that it is highly conserved with an ORF of 738 bp in size with $>99 \%$ percentage identity and $>96 \%$, both at nucleotide and amino acid levels, within species and between species, respectively. Phylogenetic analysis displayed the distinct clusters of members of genus Capripoxvirus as GTPV, SPPV and LSDV along with various species-specific signature residues which may be useful in genotyping of capripoxviruses. Despite different host specificity of poxviruses, comparative sequence analysis of L1R proteins revealed highly conserved nature with presence of myristoylation motif (G-X-X-X-S) and six cysteine residues among all poxviruses. To the best of authors' knowledge, it is the first report on genetic characterization with protein modeling of CaPV-L1R. The conserved and immunogenic nature of L1R gene of capripoxviruses may prove to be a potential candidate for developing molecular diagnostics including recombinant protein based assays and prophylactics for control of these diseases in tropical countries like India.

\section{Inhibition of Newcastle disease virus replication by chicken viperin}

\section{Manisha Shah, Sachin Kumar}

Email ID for Correspondence: shahmanisha127@gmail.com Department of Biosciences and Bioengineering, Indian Institute of Technology Guwahati, Guwahati, Assam 781039, India

The identification of immune pathway which protects against pathogens may lead to novel molecular therapies for both livestock and human health. Viperin is one of the interferon stimulating genes (ISG) that is highly conserved from lower vertebrates to mammals and plays an important role in protection against virus. Viperin and other ISGs orthologues are not well studied in context of virus infection in chickens. Newcastle disease virus (NDV) is the causative agent of a Newcastle disease, which is economically important for avian species. NDV is endemic in many developing countries including India. NDV outbreaks have been reported because of the emergence of variant strains. In the present study, we cloned the chicken Viperin (cViperin) gene from peripheral blood mononuclear cells and analyzed its modulation upon NDV infection in chicken embryo fibroblast (CEF). Overexpression of cViperin showed inhibition of NDV replication in CEF. The cViperin gene expression down regulated the expression of NDV protein genes. Similarly, protein expression studies showed significant down regulation of NDV in presence of cViperin. The findings in the study indicated the anti-NDV effect of cViperin which could be helpful in the development of antiviral therapies against its infection.

Development of an alternate potency test for quality control of classical swine fever vaccines

Manu M, Surendra Kumar Badasara, Richa Pachauri, Vikramaditya Upmanyu, Ashok Kumar Tiwari and Pronab Dhar

Email ID for Correspondence: manumohankrishna123@gmail.com Division of Biological Standardization, ICAR-Indian Veterinary Research Institute, Izatnagar, Bareilly, Uttar Pradesh, India-243122 
Classical Swine Fever (CSF) is one of the most important diseases of pigs in terms of morbidity and mortality. The disease is caused by Classical Swine Fever virus (CSFV) of genus Pestivirus, family Flaviviridae. Effective vaccines are available for control of the disease and potency test of the CSF vaccines is of utmost importance to ensure that the vaccines available for use are of standard quality. A CSF vaccine is said to be of standard quality if it contains at least 100 PD50 virus/dose. Currently the 50\% Protective Dose (PD50) of CSF vaccines are determined by pig challenge experiments causing the controls and even some vaccinated animals to suffer from the infection leading to death. In the present study, a Fluorescent Antibody Virus Neutralization (FAVN) test has been developed and based on this test, an alternate potency test has been developed in which the 28 days post vaccinated pig serum are evaluated by FAVN test and PD50/dose is calculated based on the FAVN titre without requiring the animals to be challenged. The correlation between the FAVN titre and animal protection against challenge has been established and the minimum FAVN titre for protection against CSF disease was found to be 10. Seven batches of CSF vaccine have been tested by FAVN in parallel with challenge experiment, and our study on 105 serum samples revealed that FAVN titre matches $100 \%$ with the protection. Thus, the in vitro method (determination of PD50 by FAVN) developed in the present study can do away the challenge of vaccinated and control animals, thus avoiding the sufferings of animals in potency testing of the vaccine.

Defective interfering (DI) particles of peste-des-petits ruminants (PPR) vaccine virus: implications in vaccine up-scaling

\section{Mousumi Bora ${ }^{1}$, Raja Wasim Yousuf ${ }^{1}$, Pronab Dhar ${ }^{2}$ Rabindra Prasad Singh ${ }^{1}$}

Email ID for Correspondence: mousumeebora11@gmail.com ${ }^{1}$ Division of Biological Products, ICAR-Indian Veterinary Research Institute, Izatnagar, Bareilly, Uttar Pradesh, India-243122; ${ }^{2}$ Division of Biological Standardization, ICAR-Indian Veterinary Research Institute, Izatnagar, Bareilly, Uttar Pradesh, India-243122

Defective interfering (DI) particles are non-infectious deletion mutants which arise as a result of repeated passages of virus at high multiplicity of infection (MOI). Generation and rapid accumulation of such defective particles in cultured cells lead to slowing down of the parent virus population. Higher MOIs attribute to earlier and faster decline in virus levels. The vaccine strain of Peste-des-petits ruminants virus, "PPRV Sungri/96" is presently been extensively used by several commercial firms and government establishments for production of PPR vaccines in India and often, manufacturing of such live attenuated vaccines show instances of low titres. In the present study we generated DI particles of PPRV Sungri/96 vaccine virus using Vero cells at three different MOIs viz. 0.5, 1 and 3, including the standard low MOI (0.01). Selecting each MOI, serial undiluted passages were made in cultured cells. Detailed pattern of cytopathic effects and infectivity titres of virus in each passage were recorded. Antigen based detection of PPRV specific DI particles were also done with Cell ELISA and Indirect Immunofluorescence test using monoclonal antibodies against nucleocapsid (clone 4G6) and hemagglutinin (clone 4B11) protein of PPR virus. The investigations revealed significant decline of virus titre from $10^{6.5}$ to $10^{3.5} \mathrm{TCID}_{50} /$ $\mathrm{ml}$ with undiluted virus samples propagated with high MOI, whereas no such effect was observed with the undiluted virus propagated with low MOI (MOI 0.01). Investigation on generation of PPRV specific DI particles will be useful to find out a safe MOI for propagation of vaccine viruses in cell culture based system and will help in further up-scaling of PPR vaccines without hindrance in view of National and Global PPR control and eradication.
Understanding the expression modulation of non-structural viral proteinW of Newcastle disease virus

\section{B. Nagaraj Nayak, Saraswathy P. V. Madhuri Subbiah}

Email ID for Correspondence: bnagraj.nayak@gmail.com National Institute of Animal Biotechnology, Hyderabad, Telangana, India - 50049

Newcastle disease virus (NDV)is an endemic poultry pathogen in India. NDV belongs to serotype 1 of avian paramyxoviruses of genus Avulavirus within family Paramyxoviridae. NDV has a negative sense, single stranded, non-segmented RNA genome ( $\sim 15 \mathrm{~kb})$. The genome carries six genes arranged in tandem, coding for nucleocapsid protein, phosphoprotein $(\mathrm{P})$, matrix protein, fusion protein, hemagglutinin-neuraminidase protein and large polymerase protein. Additionally by co-transcriptional editing of $\mathrm{P}$ gene by stuttering polymerase, two non-structural proteins, $\mathrm{V}$ and $\mathrm{W}$, are expressed. The $\mathrm{P}, \mathrm{V}$ and $\mathrm{W}$ proteins are amino co-terminal, differing at their carboxyl termini in both length and amino acid composition. Both, $\mathrm{V}$ and $\mathrm{W}$ mRNA are expressed in low quantities (29 and 2\%) and only during active viral replication in the host indicating that their expression might be highly regulated. $\mathrm{V}$ protein has been identified as interferon antagonist but nothing is known about the function of $\mathrm{W}$ protein. Our preliminary studies showed $\mathrm{W}$ protein localizing in the nucleus. Over expression of $\mathrm{W}$ protein had insignificant effect on the viral replication, however, we observe morphological changes in the $\mathrm{W}$ transfected cells suggesting apoptosis. Although an avian pathogen, NDV can infect human cells and replicate specificallywell in human cancer cells. Owing to its oncolytic property, NDV is viewed as a potential anti-cancer agent. NDV mediates oncolysis by causing apoptosis of the infected cells. Several researchers have shown the role played by multiple viral proteins in induction of apoptosis. Our studies are attempted in answering the questions on why and how the viral modulation of non-structural viral protein, W's expression, is attributing to the oncolytic property of NDV.

Comparative efficacy of recombinant protein and dsRNA against white spot syndrome virus (WSSV) infection in shrimp

Puneeth. T.G., ${ }^{1}$ Akhila, D.S., ${ }^{2}$ Girisha, S.K. ${ }^{1}$, Santhosh, S.K. ${ }^{2}$, Suresh T. and Venugopal, M.N. ${ }^{1}$

Email ID for Correspondence: puneethgowda615@gmail.com ${ }^{1}$ Depatment of Fisheries Microbiology, College of Fisheries, Mangalore- 575 002; ${ }^{2}$ Nitte University Centre for Science Education and Research, Paneer Campus, Deralakatte,, Mangaluru, 575018

White spot syndrome virus (WSSV) is a key concern in shrimp culture due to its pathogenesis and economic loss to shrimp production. RNA interference (RNAi) is a natural conserved mechanism to combat viral infections. In the present study, a ws 477 gene is targeted to synthesis recombinant protein and dsRNA to evaluate their potency against WSSV infection in shrimp, Penaeus monodon. Molecules of synthesized recombinant protein and dsRNA were administrated via injection and oral route. Shrimps injected with $w s v 477$ recombinant protein and dsRNA provided survival rates of 70 and 90 respectively. Shrimps fed with wsv 477 recombinant protein and dsRNA showed survival rates of 60 and 80 respectively. Among the protective agents, dsRNA was proved to be highly potent compared to the recombinant protein against WSSV infection. Results of this study provided evidence that the targeted WSSV gene exhibit antiviral response since it plays a crucial role in WSSV replication. Hence, this gene can be considered as the effective target in the 
development of therapeutics for the control of WSSV infection in shrimps.

\section{Sub-cellular localization and in vitro antiviral activity of transiently expressed MAVS orthologue from Asian seabass}

\author{
Rahul Krishnan, P. Gireesh Babu, Jeena K., Shyam K.U., \\ Kurcheti Pani Prasad
}

Email ID for Correspondence: rkcapricon@gmail.com Aquatic Environment and Health Management Division, ICARCentral Institute of Fisheries Education, Mumbai - 400061, India

The innate immune signaling adapter, mitochondrial antiviral signaling protein (MAVS) coordinates the signals received from two independent RLRs (RIG-1 and MDA5) to induce IFN and ISGs. In the present study, we report identification of a fish MAVS orthologue from Lates calcarifer (LcMAVS) and its functional role in fish RLR signaling. The complete cDNA sequence of $L c$ MAVS encodes a poly peptide of 577 amino acids with an N-terminal CARD-like domain, central proline-rich domain and a C-terminal transmembrane domain. The $L c$ MAVS-ORF- 2 was cloned into pcDNA3.1 and transfected into SISS cells. Single band of $61 \mathrm{KDa}$ of LcMAVS was detected in western blot and confocal microscopy demonstrated the mitochondrial localization of LcMAVS. In addition, pcDNA-MAVS transfected cells were protected against Nervous Necrosis Virus (NNV) infection as manifested by the delayed appearance of cytopathic effect (CPE) and decreased viral transcript levels. Over expression of $L c$ MAVS resulted in activation of an ISRE-containing promoter (52 folds over control cells) as well as transcriptional expression of IRF-3, IFN-1 and IFN-inducible genes including Mx and ISG15 $(P<0.05)$. These results suggest that LcMAVS is involved in the antiviral immunity as a one of the adaptors in fish IFN-activation pathway.

\section{Abortion in an organized dairy farm: an investigation}

Supriya $\mathrm{P}^{2}$, LN Sarangi ${ }^{1}$, SK Rana ${ }^{3}$, VS Bahekar ${ }^{1}$, A Prasad ${ }^{1}$, KSNL Surendra $^{1}$, RVC Reddy ${ }^{1}$, NM Ponnanna ${ }^{1}$, GK Sharma ${ }^{3}$, VA Srinivasan ${ }^{4}$

Email ID for Correspondence:

lnsarangi@nddb.coop/surendra@nddb.coop

${ }^{1}$ National Dairy Development Board Research and Development

Laboratory, IIL Campus, Hyderabad 500032, Telangana, India;

${ }^{2}$ Centre for Biotechnology, Jawaharlal Nehru Technological

University, Hyderabad 500085, Telangana, India; ${ }^{3}$ National Dairy

Development Board, Anand 388001, Gujarat, India; ${ }^{4}$ House No. 33,

Telecom Nagar House No. 33, Gachibowli, Hyderabad 500032,

Telangana, India

Bovine abortion is one of the major problem in dairy industry resulting in enormous economic loss. The involvement of multi-factorial agents in the abortion makes it difficult to identify the actual causative agent so as to initiate effective control measures. The study was conducted in an organized dairy farm of Telangana, India housing about 900 high yielding dairy cows in a stall-fed intensive rearing system with average milk production of $18 \mathrm{~kg} /$ day/milking cow. In the farm, the animals are regularly vaccinated for foot-andmouth disease, haemorrhagic septicaemia, black quarter, theileriosis, brucellosis and also for IBR from October, 2016. The farm has recorded high abortion rates (average 34.92\%) from August 2016 which impeded the productivity. A pilot study was initiated to investigate the presence and circulation of major abortion causing agents viz., Brucella, bovine herpesvirus-1 (BHV-1), Bovine viral diarrhoea (BVD), Neospora caninum, Coxiella burnetii and Leptospira hardjo in the farm. The animals $(\mathrm{n}=240)$ were bled twice at 1 year interval (August 2016 and 2017) and serum samples were screened by commercially available ELISA kits. Further, 59 abortion cases (August 2016-August 2017) submitted to the laboratory, were investigated for detection of causative agent by employing cultural, serological and molecular methods for six infectious agents. The results indicate circulation of six six infectious agents in the farm with Brucella being the major abortion causing agent in the farm followed by BHV-1. Further investigation is required on involvement of other infectious agents which has been detected serologically (Neospora, Coxiella and Leptospira) in the abortion cases by employing molecular methods. The results of the seroligical and the molecular testings will be discussed in the poster in detail.

\section{Identification of the missing link to answer the multi- functionalities of non-structural $\mathrm{V}$ protein of Newcastle disease virus}

\section{Saraswathy P.V, Sunny Deval and Madhuri Subbiah}

Email ID for Correspondence: vani.saraswathy@gmail.com National Institute of Animal Biotechnology, Hyderabad, Telangana, India - 500049

Newcastle disease virus (NDV) Komarov strain causes the most economically draining Newcastle disease (ND) in more than 250 bird species across the globe. NDV causes frequent disease outbreaks despite strict vaccinations and is a threat to poultry industry in India. NDV belongs to serotype 1 of avian paramyxoviruses of genus Avulavirus within family Paramyxoviridae. NDV has a negative sense, single stranded, non-segmented RNA genome ( $\sim 15 \mathrm{~kb})$. The genome carries six genes arranged in tandem, coding for nucleocapsid protein, phosphoprotein, matrix protein, fusion protein, hemagglutinin-neuraminidase protein and large polymerase protein. Additionally by co-transcriptional editing of $\mathrm{P}$ gene, two non-structural proteins, $\mathrm{V}$ and $\mathrm{W}$, are expressed during viral infections. RNA editing involves insertion of non-templated $\mathrm{G}$ residue(s) by stuttering polymerase. The unedited mRNA codes for $P$, while insertion of one $\mathrm{G}$ residue codes for $\mathrm{V}$ mRNA and insertion of two Gs generates $\mathrm{W}$ mRNA, with frequencies of approximately 68,29 and $2 \%$. The P, V and $\mathrm{W}$ proteins are amino co-terminal, differing at their carboxyl termini in both length and amino acid composition. The 239 amino acids long (MW: $36 \mathrm{KDa}$ ) V protein of NDV is known to be multifunctional, plays a direct role in virus replication, is a host range determinant, is anti-apoptotic and serves as a virulence factor and interferon (IFN) antagonist. However, how it does these is largely unknown. Through our confocal studies, we have visualized for the first time the replicative intermediate of NDV (dsRNA) associated with $\mathrm{V}$ protein as discrete foci around the nuclear membrane in NDVinfected cells. We speculate that $\mathrm{V}$ protein acts as a shield for dsRNA (an important trigger of IFN response) and thus supports viral replication and protects from/delays the induction of antiviral response.

\section{Characterisation of viscerotropic velogenic Newcastle disease} virus isolates

Sarika $\mathbf{N}^{1}$, John Kirubaharan ${ }^{2}$, Ranjani Rajasekaran ${ }^{3}$, Shilpa $\mathrm{P}^{4}$, Daniel Joy Chandran ${ }^{5}$

Email ID for Correspondence: sarikanavayath@yahoo.co.in 
${ }^{1} \mathrm{Ph} . \mathrm{D}$ Scholar, Department of Veterinary Microbiology, College of Veterinary and Animal Sciences, Mannuthy, Thrissur, Kerala; ${ }^{2}$ Professor and Head, Department of Veterinary Microbiology, Madras Veterinary College, Chennai; ${ }^{3} \mathrm{Ph} . \mathrm{D}$ Scholar, Department of Veterinary Microbiology, Madras Veterinary College, Chennai; ${ }^{4}$ Veterinary surgeon, Animal husbandary Department, Kerala; ${ }^{5}$ Professor (Retd.), Department of Veterinary Microbiology, Madras Veterinary College, Chennai

Newcastle disease (ND) is a highly contagious disease of poultry. Of different infectious diseases affecting poultry, this forms the major hurdle in achieving the much projected two digit growth rate in Indian poultry industry. In this context, the present study was conducted to find out the pathotype, genotype and evolution of two isolates of NDV namely D165 and D162, which have been reported to cause high mortality among backyard poultry. These isolates were pathotyped using mean death time (MDT), intracerebral pathogenicity index (ICPI), infection of maternal antibody negative chicks and molecular pathotyping by finding out the deduced amino acid sequence of fusion protein cleavage site (FPCS). To find out the genotype the whole genome was amplified and phylogenetic analysis was performed by adopting maximum likelihood algorithm using the genes responsible for virulence namely fusion protein (F), haemagglutinin-neuraminidase $(\mathrm{HN})$ and large protein $(\mathrm{L})$. The evolutionary relationship was also analysed by estimating the distance using maximum likelihood algorithm. Both the NDV isolates D165 and D162 were estimated to have a MDT time of $48 \mathrm{~h}$ and an aminoacid sequence ${ }^{112}{ }^{R R R K R F}{ }^{117}$ at the FPCS site. The ICPI values estimated for these isolates were 1.80 and 1.77 respectively. They also produced discrete ulcers throughout the intestine, petechial haemorrhages at the papillae of the proventriculus and haemorrhage at ileo-caecal junction. Based on phylogenetic trees developed and analysis of evolutionary distance the isolates were positioned in subgenotype XIIIb under genotype XIII. The virtual restriction map developed using partial $\mathrm{F}$ gene sequence against restriction enzymes HinfI, BstOI and RsaI using Restriction mapper shows that the isolates have a unique restriction pattern unlike other members of genotype XIII. In conclusion, the two isolates of NDV were reported to be of viscerotropic velogenic type belonging to genotype XIII and subgenotype XIIIb, which are emerging in India.

\section{Viability of WSSV in shrimp pond sediments with reference to physicochemical properties}

S. Satheesh Kumar, R. Ananda Bharathi, V. Chitra, J.J.S. Rajan, S.V. Alavandi

Email ID for Correspondence: satheeshgene@gmail.com ICAR-Central Institute of Brackishwater Aquaculture, Chennai - 600 028, India

Shrimp aquaculture is one of the rapidly growing industries in seafood sector. The white spot syndrome virus (WSSV) is the causative agent of white spot disease (WSD) in shrimp and has emerged as a single most critical pathogen causing severe epizootics and significant economic loss to the global shrimp aquaculture industry. It can be transmitted vertically from infected broodstock to postlarvae and horizontally by cannibalism and environmental sources such as live carriers, water and sediment. Currently, there is no therapeutic measure to control the devastating disease in aquaculture. Biosecurity is the most important means of disease management strategies in shrimp aquaculture. Shrimp pond sediment represents an important reservoir of WSSV. The viability of WSSV in sediment is the most vital parameter in the transmission of WSD in the freshly stocked ponds. The physicochemical properties of sediment also influence the viability and infectivity of WSSV. The objective of this study was to understand the viability of WSSV with reference to the physicochemical properties of pond sediment under field conditions. The sediment samples from three WSSV affected ponds with different soil texture were analyzed for viability of WSSV using polymerase chain reaction (PCR), quantitative PCR and infectivity studies. The soil characteristics such as texture, $\mathrm{pH}$, organic carbon (OC), electrical conductivity (EC), and moisture content were recorded. The WSSV was found to be detectable in shrimp pond sediment by PCR up to 35-52 days post-harvest but remained infective only up to 21-32 days post- harvest. The study also revealed that the WSSV was infective up to 21 and 25 days in sandy clay loam soil and 32 days in sandy loam soil, suggesting that the infectivity of WSSV was prolonged in sandy loam soil than the sandy clay loam soil. Further, the viability of WSSV was found to be dependent on the moisture content of the pond sediment and independent of organic carbon, $\mathrm{pH}$, and electrical conductivity of pond sediment. These observations reinforce the requirement of adoption of best management practices (BMPs) with regard to pond preparation/sun-drying of ponds for at least 3-4 weeks prior to beginning the subsequent crop to achieve biosecurity of aquaculture pond environment.

\section{Biocontrol of pathogenic Vibrio parahaemolyticus: a bacteriophage consortium}

\section{Shruthi Seetharam Shetty, Juliet MohanRaj, Krishna Kumar Ballamoole, Deekshit VK, Anirban Chakraborty, Indrani Karunasagar and Praveen Rai}

Email ID for Correspondence: kshanaalva8@gmail.com Nitte University Centre for Science Education and Research, Nitte University, Deralakatte, Mangaluru, Karnataka, India-575018

$V$. parahaemolyticus is a Gram-negative, halophilic bacterium that occurs as natural inhabitant of marine and estuarine environment. It is considered as an important pathogen for shrimp aquaculture industry leading to a substantial mortality and economic loss. The rapidly expanding shrimp aquaculture industry in India requires careful management to make it sustainable. However, there has been emergence of multiple antibiotic resistance in this bacterium due to the misuse of antibiotics to control infections. To accomplish this, biocontrol strategies such as bacteriophage therapy against $V$. parahaemolyticus would be an effective alternative to antibiotics in shrimp culture systems. With this background, the present study was initiated to isolate and characterize lytic phages against pathogenic $V$. parahaemolyticus which causes mass mortalities in penaeid shrimp. A total of $150(\mathrm{n}=150) \mathrm{V}$. parahaemolyticus were isolated from hepatopancreas, haemolymph of the moribund and healthy animals and their culture pond water samples from both West and East coast of India. All together $15 \mathrm{~V}$. parahaemolyticus phages were also isolated from the same source. All these phages were then tested for their lytic activity against $50(\mathrm{n}=50)$ different $V$. parahaemolyticus strains isolated in this study. Among them, four lytic phages ParA, ParB, ParC, ABW $(n=4)$ with varied morphology and wide host range were then propagated and characterized based on their RAPD profiles. Optimal Multiplicity of infection (MOI) was determined for one of the phages, ABW $(n=1)$ to assess the phage bacterium ratio to be applied in the phage therapeutic experiments. In addition, virion morphology of isolated phages was determined by Transmission Electron Microscopy (TEM). 
Standardization of SYBR green chemistry-based quantitative real-time PCR assay to detect infectious myonecrosis virus (IMNV) from infected shrimp tissues

\section{Shyam K U, Rahul Krishnan, Jeena K and Kurcheti Pani Prasad}

Email ID for Correspondence: shyam.aahpa603@cife.edu.in Aquatic Environment and Health Management Division, ICARCentral Institute of Fisheries Education, Mumbai-61

Indian penaeid shrimp aquaculture has recently witnessed the occurrence of infectious myonecrosis virus (IMNV) belonging to the class of dsRNA viruses. Early and precise disease diagnostics is in limelight for detecting the viral diseases. The viral load determination in infected individuals has become the most significant step to diagnose and manage shrimp viral diseases especially with regard to the detection of viral infection in asymptomatic individuals. In this study, a quantitation method for IMNV was developed using real-time PCR with SYBR Green chemistry. The specific qPCR primers were designed from major capsid protein (MCP) of ORF1 region of IMNV genome. Linearity of detection of the primers were tested using a conventional PCR format. A 135-bp PCR amplicon was cloned in pTZ57 R/T vector and the plasmid copy number was estimated. A tenfold serial dilution of the plasmid DNA from $1 \times 10^{10}$ copies to 10 copy was prepared and used as the standard. The absolute quantitation of the positive plasmid containing MCP fragment was analyzed from standard curve. The limit of detection of the assay was found to be 100 copies of plasmid. The designed SYBR Green assay were found to be sensitive and specific with a wide dynamic range $\left(1 \times 10^{10}\right.$ to 10 copies) with coefficient of regression (R2) $>0.97$ and calculated average slope -3.5834 . Inter-assay variations were ruled out by using a single series of dilution, run in three assays. Single specific amplification was ascertained by melt curve analysis. Validation of the standardized assay is being carried out using IMNV infected shrimp samples collected from infected farms.

Genome segment 2 encoded RNA polymerase gene based haracterization of camelid origin picobirnaviruses, India

Subhankar Sircar ${ }^{1}$, A.K. Verma ${ }^{1}$, J.K. Kala ${ }^{2}$, A.B. Joshi ${ }^{2}$, H.C. Chauhan ${ }^{2}$, B.S. Chandel ${ }^{2}$, S.K. Mohapatra ${ }^{2}$ and Yashpal S. Malik ${ }^{1}$

Email ID for Correspondence: shubhankar.sircar@gmail.com

${ }^{1}$ ICAR-Indian Veterinary Research Institute, Izatnagar- 243 122,

Bareilly (U.P); ${ }^{2}$ College of Veterinary Science and AH,

Sardarkrushinagar Dantiwada Agricultural University,

Sardarkrushinagar- 385506 (B.K.), Gujarat, India

Camels are considered unique because of their adaptability to exacting desert conditions where they withstand high temperatures and a shortage of food and water. Due to these capabilities, camels have been used by the mankind since time $3000 \mathrm{BC}$ for transportation, military uses, and as a source of meat, milk and wool. Though camels pose less danger to humans their close association to humans can intensify the threat of zoonotic pathogens. The latest findings of MERS along with other families of viruses viz., Paramyxoviridae, Flaviviridae, Herpesviridae in different reports from around the world has increased the concern of researchers regarding the viral emergencies in camelids. Here, we worked to diagnose Picobirnavirus (PBVs) in Kutchhi breed camel fecal samples $(\mathrm{n}=29)$ from the Dhori District, Bhuj, Gujrat. The PBVs are among the emerging enteric viral pathogen, composed of dsRNA of bipartite nature. It has been identified as a major pathogen associated with diarrhoeal illness in humans and animals. We could diagnose the presence of PBV in
93.1\% (27/29) samples using RdRp gene based RT-PCR. Further, three positive samples were randomly selected and sequenced near to full length region (1.2 Kbp) of genomic segment 2 encoding for RdRp gene. Upon sequence analysis of the RdRp genes from these PBV isolates we observed huge diversity among them and all three isolates clustered in different clades consisting of other species sequences like human, dromedary, porcine etc. Although the isolates were sequenced from a single site they exhibit considerable diversity among each other which points towards the ongoing reassortment events among the different species of PBVs. These findings points toward that these zoonotic viral species could someday emerge as a potent threat to the animals as well as to humans because reassortment and recombination events in segmented viruses can give rise to highly pathogenic strains of greater significance.

\section{Characterization of Newcastle disease virus expressed Erns protein of CSFV}

\section{Vishnu Kumar, Rakesh Kumar, Sachin Kumar}

Email ID for Correspondence: vcthakur42@gmail.com Department of Biosciences and Bioengineering, Indian Institute of Technology Guwahati, Guwahati 781039 Assam India

Newcastle disease virus (NDV) has been used as a virus vector to express different proteins successfully. NDV can be grown in embryonated chicken eggs. Therefore, this is very easy way to produce any foreign protein. Here, we constructed a recombinant NDV that express the $\mathrm{E}^{\mathrm{rns}}$ protein of Classical swine fever virus (CSFV). The structural glycoprotein $\mathrm{E}^{\mathrm{rns}}$ assists in the initial attachment of the virus to the host cell and its subsequent entry. In addition, $\mathrm{E}^{\mathrm{rns}}$ protein also has Ribonuclease (RNase) activity. In the present work, we characterized the RNase activity of recombinant NDV expressing $E^{\text {rns }}$ of CSFV. In future, this recombinant NDV expressing $\mathrm{E}^{\text {rns }}$ can be used as a diagnostics for classical swine fever.

\section{Young Scientist Award}

Genome-wide comparative transcriptome landscape of rice (Oryza sativa L.) provides novel molecular insights into stress dynamics of the plant against Rice tungro viruses

\section{Gaurav Kumar, Indranil Dasgupta}

Email ID for Correspondence: gauravkumar.bhu@gmail.com Department of Plant Molecular Biology, University of Delhi South Campus, New Delhi, India-110021

Rice tungro is among the most damaging viral disease of rice resulting from infection by two viruses: Rice Tungro Bacilliform Virus and Rice Tungro Spherical Virus through the green leafhopper (Nephotettix virescens) vector. The RNA sequencing technique has completely revolutionized the otherwise microarray based transcriptome studies providing an excellent platform for gene expression analysis. The overall objective of this study is to increase our understanding towards the stress response dynamics of the rice plant upon Tungro infection and identify potential candidate genes for improved crop resistance against Tungro. In order to gain molecular insights and to comprehend the intricacies of the gene regulatory networks associated with Tungro infection, the whole genome transcriptome study was performed comparing the Tungro infected and un-infected plants using Illumina Hiseq 2500. This generated around $10 \mathrm{~GB}$ of sequenced data comprising about 50 million paired end 
reads per sample which were then aligned on to the rice genome version MSU 7.0. Gene Ontology (GO) enrichment analysis was performed using BiNGO plug-in at the java based Cytoscape platform and Blast2GO software, which revealed important GO categories under plant cellular and metabolic processes. Gene expression analysis revealed around 959 transcripts to be differentially expressed. The data was validated through qRT-PCR. The differentially expressed genes were then mapped to the predefined metabolic pathways available in Gramene Ricecyc database and MapMan software. The data revealed many important defense related genes and their associated metabolic pathways to be affected upon Tungro infection.

\section{Prognostic significance of multiple HPV genotype infection} in invasive cervical carcinoma: a report from Odisha, India

\section{Rashmirani Senapati ${ }^{1}$, Bhagyalakhsmi Nayak ${ }^{2}$, Ambarish Dutta ${ }^{3}$, Bhagirathi Dwibedi $^{1}$}

Email ID for Correspondence: rasmisenapati@gmail.com

${ }^{1}$ Viral research and diagnostic laboratory, Regional Medical research centre, Chandrasekharpur, Bhubaneswar-751023, Odisha; ${ }^{2}$ Acharya Harihara regional cancer centre, Cuttack, Odisha; ${ }^{3}$ Indian institute of public health, Bhubaneswar-751024, Odisha

Cervical cancer is the fourth most common cancer in women worldwide. It is well known that high-risk HPV is the main etiological agent for this infectious viral carcinoma. In vitro studies suggest that the presence of HPV derived E6 and E7 proteins correlates with reduced radiosensitivity. Little is known about the impact of HPV on the response to radiotherapy and on the patients' clinical outcome. To determine the association of different HPV genotypes with the survival period of invasive cervical carcinoma patients treated with radiotherapy, 210 invasive cervical carcinoma cases were enrolled from Acharyaharihara regional cancer centre, Cuttack, Odisha. Clinical history, and cervical sample was collected, DNA was isolated and HPV was detected from all the samples. 15 HPV genotypes were detected by E6/E7 multiplex nested type-specific PCR and sequencing in HPV-positive cervical samples. The patients were followed-up for their treatment, recurrence, and death status. Frequency distributions, Progression-free survival (PFS), Cervical cancer-specific survival, Survival analysis and Survival curves were calculated were calculated using standard methods and the results were statistically analyzed using appropriate statistical tools. 197 (93.80\%) cases were HPV-positive. A total of 182 cases were included in the analysis. 132 (62.9\%) cases were infected with Single HPV genotypes which includes HPV 16, HPV 18 and HPV45. 26 Cases infected with double genotypes which includes HPV16 + 18, HPV16 + 44, HPV $16+35$, HPV $16+35$, HPV $16+39$, HPV $18+39$, HPV $18+51$, HPV18 + 45 genotypes combination. $14(6.7 \%)$ cases are detected with Triple and quadruple infections which includes the genotypes combinations such as HPV16 + 66+68, HPV16 + 66 + 51, HPV $16+18+39, \quad$ HPV $16+18+58, \quad$ HPV $58+35+42$, HPV $16+51+52+42$, HPV $16+6 / 11+43$ and HPV $18+45$ $+44+51$. Hazard ratio was estimated to be 1.3 (95\% CI 6.5-2.62) for single vs. double genotypes and 2.5 (95\% CI 1.18-5.6) for single vs. more than double genotypes ( $\log \operatorname{rank} 6.2, P=0.04$ ). Patients with double HPV infection and more than double infection had a significantly shorter disease free survival compared to those with single HPV infection. Our results demonstrate that the presence of multiple HPV types is associated with low survival period. This is the preliminary result for the association of only HPV genotypes with survival. Association study of other clinical variable with a multivariate analysis is ongoing which will provide more clarity to the result.
Epidemiological data and computational prediction confirms genetic predisposition to rotavirus infection: need of precision immunization

Kshitija Suhas Rane-Yadav ${ }^{1}$, Selvaa Kumar $C^{2}$, Dattatraya. S. Joshi ${ }^{3}$, Nimain C Mohanty ${ }^{4}$, Nitin N Kadam ${ }^{4}$

Email ID for Correspondence: Kshitijarane@ymail.com ${ }^{1}$ Department of Medical Genetics, MGMIHS OMICS Research Center, MGM Institute of Health Sciences, Navi Mumbai, India410209; ${ }^{2}$ Department of Bioinformatics, Institute of Biotechnology and Bioinformatics, D Y Patil university, Belapur, Navi Mumbai400614; ${ }^{3}$ Department of Medical Genetics, MGM Institute of Health Sciences, Kamothe, Navi Mumbai, India-410209; ${ }^{4}$ Department of Pediatrics, MGM Institute of Health Sciences, Kamothe, Navi Mumbai, India-410209

In 2013, India contributed $22 \%$ of global mortality caused by Rotavirus. Lack of therapy and efficient vaccine is the prime reason for this burden. On the basis of surface viral protein protein VP4 (VP5 + VP8) there are various P-genotypes of rotavirus. Current immunization programme includes Rotarix, Rotateq $(\mathrm{P}[8])$ and Rotavac (P[11]). Specificity of various rotavirus genotypes to unique geographical region may show strain specific and host specific variation. Therefore, vaccines currently in use may not be beneficial or necessary for part of the population. Clinically suspected 100 children $<5$ years of age (U5C) suspected for viral diarrhea were screened for rotavirus infection. Lewis antigen expression in healthy individuals was compared with rotavirus positive and negative cases of diarrhea. EDTA blood from selective cases was processed for fucosyltransferase-2 (FUT2) gene sequencing. Role of FUT2 gene in rotavirus infection was further confirmed by RNA sequencing of surface spike protein VP8; followed by 3D protein modeling and molecular docking with Leb oligosachharide. Out of 100, 17 children were rotavirus positive. Red blood cell (RBC) phenotyping of control healthy individuals for Leb antigen indicated high frequency (16\%) of nonsecretor individuals who lack secretory Lewis antigens. NonRotavirus (NRV) diarrhea cases showed similar results (15.27\%), unlike Rotavirus-positive (RV) cases of $\mathrm{P}[4]$ and $\mathrm{P}[8]$ genotype which altogether were found to show the presence of Leb antigen (secretor status), $0 \%$ non-secretors. Association of $\alpha 1 \rightarrow 2$ fucosylated blood group antigen with rotavirus infection is found to be statistically significant $\left(\chi^{2} 0.04\right)$. No significant difference was found in healthy and NRV (p-value 0.24). Rotavirus positive cases (RV) were significantly associated with presence of at least single wild type allele at both loci i.e. FUT2 ${ }^{302+/+}(86.66 \%)$ or FUT2 ${ }^{302+/-}(13.33 \%)$ and FUT2 ${ }^{428+/+}(80 \%)$ or FUT2 ${ }^{428+/-}$ (20\%). Difference of genotypes AA-FUT2 $2^{428-l-}$, GA-FUT2 $2^{428-1+}$, TT-FUT2 $2^{302-1-}$, CT-FUT2 $2^{302-l+}$ between the group of non secretors ( $\mathrm{Le}^{\mathrm{b}}$ absent) and rotavirus positive cases was found to be statistically significant (C302T: $\chi^{2} 0.018$; G428: $\chi^{2}$ 0.067). P[4] genotype was found to be homologous to P[8] genotype at RNA as well as protein sequence level ( $99 \%$ homology). Comorbidity of these genotypes was not found. P[4] and P[8] genotypes show similar active binding site and both types of proteins actively interact with Lewis b type antigens. 100D, 145G, $153 \mathrm{~N}$ and $154 \mathrm{R}$ are active residues and represent binding site of VP8 protein. Present study scientifically proves that human rotaviruses follows unique path using secretory ABO blood group antigens. Knowledge of molecular biology of the Rotavirus pathogenesis may open up new paths for vaccines and therapy. 
Peripheral blood immune cell subsets specific transcriptome unravelled early high throughput gene expression predictors of Peste des petits ruminants vaccine induced response in sheep and goats

\section{Sajad Ahmad Wani ${ }^{1}$, Amit Ranjan Sahu ${ }^{1}$, Shikha Saxena ${ }^{1}$, Aruna Pandey ${ }^{1}$, Sonam Kanchan ${ }^{1}$, Alok Khanduri ${ }^{1}$, Vaishali Sah ${ }^{2}$, Dheeraj Chaudhary ${ }^{3}$, Kaushal Kishor Rajak ${ }^{4}$ Aditya Prasad Sahoo ${ }^{1}$, Bina Mishra ${ }^{4}$, D. Muthuchelvan ${ }^{3}$, Ashok Kumar Tiwari ${ }^{5}$, Bishnu Prasad Mishra ${ }^{1}$, Raj Kumar Singh ${ }^{1}$, Ravi Kumar Gandham ${ }^{1}$}

Email ID for Correspondence: wanisajad759@gmail.com ${ }^{1}$ Division of Veterinary Biotechnology, ICAR- Indian Veterinary Research Institute, Izatnagar, Bareilly, UP-243122; ${ }^{2}$ Division of Animal Genetics, ICAR- Indian Veterinary Research Institute, Izatnagar, Bareilly, UP-243122; ${ }^{3}$ Division of Virology, ICAR-Indian Veterinary Research Institute, Mukteshwar Campus, Nainital, Uthrakhand -263138; ${ }^{4}$ Division of Biological Products, ICAR-Indian Veterinary Research Institute, Izatnagar, Bareilly, UP-243122; ${ }^{5}$ Division of Biological Standardization, ICAR-Indian Veterinary Research Institute, Izatnagar, Bareilly, UP-243122

Peste des petits ruminants is an acute, highly contagious disease of sheep and goats, caused by PPR virus belonging to the genus Morbillivirus and family Paramyxoviridae. In the present study, healthy sheep and goats, confirmed negative for PPRV antibodies by monoclonal antibody based c-ELISA and by serum neutralization test, and for PPRV antigen by s-ELISA were vaccinated with live attenuated PPR vaccine virus (Sungri/96). Vaccinated goats were seropositive by 9 days post vaccination ( $\mathrm{dpv}$ ) while in sheep idiosyncratic response in the protective anybody titre was observed between 9 and $14 \mathrm{dpv}$ for different animals. Herein, we describe transcriptional profiling of circulating $\mathrm{T}$ helper cells, $\mathrm{T}$ cytotoxic cells, monocytes, B lymphocytes and natural killer cell responses by RNA-Sequencing of PPRV vaccinated sheep and goats at 0 and $5 \mathrm{dpv}$ to decipher the vaccine induced immune response and predict early indicators of protection. $\mathrm{CD}^{+}{ }^{+}$(T-helper), $\mathrm{CD} 8^{+}$(T-cytotoxic), CD14 ${ }^{+}$(Monocytes), CD21 ${ }^{+}$ (B-lymphocytes) and CD335 ${ }^{+}$(Natural Killer) cells were enriched using MACS technology and purity of cells $(>90 \%)$ was detected by flow cytometry. Groups of differentially expressed genes like antiviral interferon-stimulated genes, chemokines, interleukins, viral sensors etc. were found transcriptionally active early vaccination. Concordance in expression of key genes between RNA sequencing and qRTPCR was found. In this presentation, the results of the transcriptome analysis will be discussed in detail with reference to expression of specific genes in different enriched cell types in goat, sheep and both sheep and goat. Gene expression panel found in this study may address the need for sensitive diagnostics for PPRV vaccine induced protection during the "window period" after vaccination prior to the appearance of a detectable antibody response.

Vector mediated transmission of tobacco streak virus in cotton ecosystem through Parthenium pollen grains

\section{Shanmuga Prema ${ }^{1}$, N. Ganapathy ${ }^{2}$, V.G. Malathi ${ }^{1}$ and P. Renukadevi ${ }^{1}$}

Email ID for Correspondence: prema.ms1990@gmail.com

${ }_{1 \& 2}$ Department of Agricultural Entomology, Tamil Nadu Agricultural University, Coimbatore - 3, India; ${ }^{1}$ Department of Plant Pathology, Tamil Nadu Agricultural University, Coimbatore - 3, India

A necrosis disease caused by tobacco streak virus (Ilarvirus) was reported in recent years in Tamil Nadu in cotton. Thrips the early season cotton pest was suspected to facilitate the transmission of TSV. Thrips palmi was observed to pre-dominate the cotton growing belts of Tamil Nadu according to survey conducted during 2015-2017. Thrips palmi population predominating cotton ecosystem was mass cultured under glass house condition in Department of Plant Pathology, TNAU, Coimbatore. The adults and 2nd instar were allowed to feed on TSV infected cotton leaves for different AAP (Acquisition access period)(12 h, $24 \mathrm{~h}, 48 \mathrm{~h}$ and $72 \mathrm{~h}$ ). The presence of the virus in adults and 2nd instars Thrips palmi was confirmed after $72 \mathrm{~h}$ of AAP by immune (DAS-ELISA, DIBA) and nucleic acid techniques (RT-PCR and Nucleic Acid Spot Hybridization (NASH). Transmission studies conducted with cotton hybrid, Ankur 3220 revealed that TSV was regularly transmitted to cotton test seedlings, when viruliferous adults of Thrips palmi were mixed with virus-carrying pollen from Parthenium hysterophorus infected with tobacco streak virus. The virus was also regularly transmitted when virus carrying pollen was placed on the leaves of cotton test seedlings and the thrips then introduced. Symptom expression was observed after 25 days of incubation. No transmission occurred when test seedlings were exposed to virus-carrying pollen in the absence of the thrips or to the thrips without pollen. Further, no transmission occurred when the thrips were fed on virus-infected cowpea leaves and then transferred to cotton test seedlings in the absence of virus-carrying pollen. The above results were confirmed serologically through DAS-ELISA and through RT-PCR. This evidence indicates that the transmission of tobacco streak virus by Thrips palmi depends on the presence of TSV infected Parthenium pollen, which presumably infects via wounds made by the thrips.

Identification and characterization of tomato spotted wilt virus in the state of Tamil Nadu; development of TSWV detection kit

\section{Senthilraja, C., Malathi, V. G., Nakkeeran, S and Renukadevi, P}

Email ID for Correspondence: csenthilraja1991@gmail.com Department of Plant Pathology, Centre for Plant Protection Studies, Tamil Nadu Agricultural University, Coimbatore-03

A tospovirus (Tomato spotted wilt virus) infecting ornamentals and vegetable has been identified in southern India since 2015. The disease affected Antirrhinum majus, Callistephus chinensis (Chinese aster), Tagetes patula (Marigold) and Solanum lycopersicum (Tomato) exhibit typical symptoms like veinal necrosis, necrotic spots and lesion on leaves, browning of flower petals, and stem necrosis typical of tospoviruses in plants from Nilgiris district of Tamil Nadu. In Triple Antibody Sandwich-Enzyme Linked immunosorbent Assay (TAS-ELISA), presence of TSWV was confirmed In host range study, most of the inoculated plant species belonging to the families Solanaceae, Fabaceae, Amaranthaceae Astraceae and Balsaminaceae expressed their symptoms on 4th-5th Day Post Inoculation for all the four isolates; tomato seedlings expressed symptoms at 10-12th DPI. Symptoms like chlorotic rings, necrotic lesion, stunting and wilting of plants were observed in all the assay hosts. Plant species including Nicotiana tabacum and $N$. benthamiana had very high concentration of virus as assessed by TAS-ELISA for all the four isolates. Among four isolates characterized by biological assay, isolate TN To (Tomato) found to be the most virulent. Full length Nucleocapsid (Nc-850 bp) protein genes for four isolates were amplified by Reverse Transcriptase-Polymerase Chain Reaction (RTPCR) and respective fragments were cloned and sequenced. The Nc gene of four study isolates showed $97-100 \%$ identity in nucleotide and amino acid level with other isolates of TSWV. Coat protein gene of TSWV was cloned in pET28a + vector and expressed in Escherichia coli (BL21) cells. Recombinant coat protein ( $29 \mathrm{KDa})$ was purified and used for immunization of rabbits (New Zealand white 
breed) to produce the TSWV-specific polyclonal antiserum. The antiserum reacted specifically in Double Antibody Sandwich-Enzyme Linked immunosorbent Assay (DAS- ELISA) in the dilution of 1:2000. The developed DAS- ELISA kit was used for the detection of TSWV in naturally infected plants. The above results will be presented.

\section{Virological and immunological responses of goatpox (GTPV) virus in goats and sheep}

\section{S. K. Minhas, M. A. Ramakrishnan, D. Chaudhary, C. Y. Pundkar, A. B. Pandey}

Email ID for Correspondence: sanjeevna86@gmail.com Division of Virology, Indian Veterinary Research Institute, Mukteswar, Uttarakhand - 263138

Sheeppox virus (SPPV) and goatpox virus (GTPV) belong to the genus Capripoxvirus (CaPV) of the family Poxviridae. Due to limited information available on CaPV strains infecting both goats and sheep, the current study was designed to compare the virological and immunological responses in both species against the GTPV. Six sheep and six goats were inoculated intradermally with $10^{3.5} \mathrm{TCID}_{50} / \mathrm{mL}$ of GTPV/animal and were observed daily for the development of clinical signs of disease. To investigate virus shedding, total DNA was isolated from blood samples, nasal and conjunctival swabs samples collected on the weekly interval for up to 63dpi and was screened by real-time PCR assay to obtain absolute viral genome copy number. Further, the humoral response was assessed by Serum neutralization test (SNT) and the cell-mediated immune (CMI) response was evaluated by alamarBlue based lymphocyte proliferation assay. The increase in mean rectal temperature $\left(>40{ }^{\circ} \mathrm{C}\right)$ was observed in both goats and sheep. The inoculated goats showed moderate to severe lesions, whereas sheep exhibited only mild lesions. Overall, the viral genetic load was elevated in a greater proportion of goats than sheep. The humoral response assessed by SNT revealed maximum titers of $1.55 \pm 0.23 \log _{10}$ and $1.15 \pm 0.16 \log _{10}$ in goats and sheep, respectively. The CMI response revealed a significant proliferative response in lymphocyte induced with ConA, PHA-P (T cell mitogens) and PWM (T and B cell mitogen) in both goats and sheep; but with
LPS (B cell mitogen) in goats only. This study indicates that GTPV used in the current study can infect both species with severe disease in goats than sheep. Further whole genome sequencing of GTPV of goat and sheep origin will shed light on the better understanding of the cross-species transmission of CaPV's.

Inhibition of West Nile virus replication by small interfering RNA targeting a highly conserved sequence

\section{Divyanshi Karothia ${ }^{1}$, Jyoti. S. Kumar ${ }^{1}$, Sameer Bhagyawant ${ }^{3}$,} P. K. Dash ${ }^{1}$, M. M. Parida ${ }^{2}$

Email ID for Correspondence: dkrothiy@gmail.com

${ }^{1}$ Division of Virology, Defence Research and Development

Establishment, Jhansi Road, Gwalior-474002, India; ${ }^{2}$ Division of

Food Microbiology, Defence Food Research Laboratory, Mysore, India; ${ }^{3}$ Department Of Biotechnology, Jiwaji University, Gwalior

West Nile virus (WNV) is an emerging human pathogen for which specific antiviral therapy has not been developed. West Nile virus is highly prevalent in India with various confirmed cases reported from Maharastra, Madhya Pradesh, Gujarat, Orissa, and Assam. Despite its high prevalence in India and other countries there is no specific treatment available to prevent West Nile virus infection. RNA interference (RNAi) is one of the effective emerging ant-viral strategies to inhibit virus infection in cells. In this study, the efficacy of a novel siRNAs against West Nile virus was established in vitro. Two siRNAs were designed against the genomic region of West Nile virus, which is highly conserved among different genotypes of WNV. The siRNA activity was evaluated by detecting both the infectious virus and its genomic RNA. Further studies including dose dependent and time course kinetics revealed that both the siRNAs were effective with significant protection up to $48 \mathrm{~h}$ post infection (hpi). These results were in agreement with the quantitative RT-PCR assay revealing similar reduction in west Nile viral genomic RNA. Besides qRT-PCR, Western blot analysis also confirmed higher antiviral response of siRNA. The expression of housekeeping gene was not affected by the siRNA, indicating no off target effects. Thus the siRNA intervention optimized in this study has the potential for therapeutic approach against emerging West Nile virus. 